\title{
REAL SUBMANIFOLDS OF MAXIMUM COMPLEX TANGENT SPACE AT A CR SINGULAR POINT, I
}

\author{
XIANGHONG GONG AND LAURENT STOLOVITCH
}

\begin{abstract}
We study a germ of real analytic $n$-dimensional submanifold of $\mathbf{C}^{n}$ that has a complex tangent space of maximal dimension at a CR singularity. Under some assumptions, we show its equivalence to a normal form under a local biholomorphism at the singularity. We also show that if a real submanifold is formally equivalent to a quadric, it is actually holomorphically equivalent to it, if a small divisors condition is satisfied. Finally, we investigate the existence of a complex submanifold of positive dimension in $\mathbf{C}^{n}$ that intersects a real submanifold along two totally and real analytic submanifolds that intersect transversally at a possibly non-isolated CR singularity.
\end{abstract}

\section{Contents}

1. Introduction and main results

2. CR singularities and deck transformations

3. Formal deck transformations and centralizers

4. Normal forms of commuting biholomorphisms

5. Real manifolds with an abelian CR-singularity

6. Rigidity of product quadrics

7. Existence of attached complex manifolds

References

\section{INTRODUCTION AND MAIN RESULTS}

1.1. Introduction. We are concerned with the local holomorphic invariants of a real analytic submanifold $M$ in $\mathbf{C}^{n}$. The tangent space of $M$ at a point $x$ contains a maximal complex subspace of dimension $d_{x}$. When $d_{x}$ is constant, $M$ is called a Cauchy-Riemann (CR) submanifold. The CR submanifolds have been extensively studied since E. Cartan [Car32], Car33], Tanaka [Tan62], and Chern-Moser [CM74].

We say that a point $x_{0}$ in a real submanifold $M$ in $\mathbf{C}^{n}$ is a $\mathrm{CR}$ singularity, if the complex tangent spaces $T_{x} M \cap J_{x} T_{x} M$ do not have a constant dimension in any neighborhood of $x_{0}$. A real submanifold with a CR singularity must have codimension at least 2 . The study

Date: June 24, 2021.

2010 Mathematics Subject Classification. 32V40, 37F50, 32S05, 37G05.

Key words and phrases. Local analytic geometry, CR singularity, normal form, integrability, reversible mapping, linearization, small divisors, hull of holomorphy.

Research of L. Stolovitch was partially supported by ANR grant "ANR-10-BLAN 0102" for the project DynPDE and ANR grant "ANR-14-CE34-0002-01" for the project "Dynamics and CR geometry". 
of real submanifolds with $\mathrm{CR}$ singularities was initiated by E. Bishop in his pioneering work [Bis65]. He investigated a $C^{\infty}$ real submanifold $M$ of which the complex tangent space at a CR singularity is minimal, that is exactly one-dimensional. The very elementary models of this kind of manifolds are the Bishop quadrics $Q_{\gamma}$ that depends on the Bishop invariant $0 \leq \gamma \leq \infty$, given by

$$
Q_{\gamma} \subset \mathbf{C}^{2}: z_{2}=\left|z_{1}\right|^{2}+\gamma\left(z_{1}^{2}+\bar{z}_{1}^{2}\right), \quad 0 \leq \gamma<\infty ; \quad Q_{\infty}: z_{2}=z_{1}^{2}+\bar{z}_{1}^{2} .
$$

The complex tangent at the origin is said to be elliptic if $0 \leq \gamma<1 / 2$, parabolic if $\gamma=1 / 2$, or hyperbolic if $\gamma>1 / 2$. In MW83, Moser and Webster studied the normal form problem of a real analytic surface $M$ in $\mathbf{C}^{2}$ which is the higher order perturbation of $Q$. They showed that when $0<\gamma<1 / 2, M$ is holomorphically equivalent, near the origin, to a normal form which is an algebraic surface that depends only on $\gamma$ and two discrete invariants. We mention that the Moser-Webster normal form theory, as in Bishop's work, actually deals with an $n$-dimensional real submanifold $M$ in $\mathbf{C}^{n}$, of which the complex tangent space has (minimum) dimension 1 at a CR singularity.

The main purpose of this paper is to investigate an $n$-dimensional real analytic submanifold $M$ in $\mathbf{C}^{n}$, which is totally real outside a proper analytic subset and of which the complex tangent space has the largest possible dimension at a given $\mathrm{CR}$ singularity. We shall say that the singularity is a (maximal) complex tangent. The dimension must be $p=n / 2$. Therefore, $n=2 p$ is even. We are interested in the normal form problem, the rigidity property, and the local analytic geometry of such real analytic manifolds.

In suitable holomorphic coordinates, a $2 p$-dimensional real analytic submanifold $M$ in $\mathbf{C}^{2 p}$ that has a complex tangent space of maximum dimension at the origin is given by

$$
M: z_{p+j}=E_{j}\left(z^{\prime}, \bar{z}^{\prime}\right), \quad 1 \leq j \leq p,
$$

where $z^{\prime}=\left(z_{1}, \ldots, z_{p}\right)$ and

$$
E_{j}\left(z^{\prime}, \overline{z^{\prime}}\right)=h_{j}\left(z^{\prime}, \bar{z}^{\prime}\right)+q_{j}\left(\bar{z}^{\prime}\right)+O\left(\left|\left(z^{\prime}, \bar{z}^{\prime}\right)\right|^{3}\right) .
$$

Moreover, each $h_{j}\left(z^{\prime}, \bar{z}^{\prime}\right)$ is a homogeneous quadratic polynomial in $z^{\prime}, \bar{z}^{\prime}$ without holomorphic or anti-holomorphic terms, and each $q_{j}\left(\bar{z}^{\prime}\right)$ is a homogeneous quadratic polynomial in $\bar{z}^{\prime}$. One of our goals is to seek suitable normal forms of perturbations of quadrics at the CR singularity (the origin).

1.2. Basic invariants. To study $M$, we consider its complexification in $\mathbf{C}^{2 p} \times \mathbf{C}^{2 p}$ defined by

$$
\mathcal{M}: \begin{cases}z_{p+i}=E_{i}\left(z^{\prime}, w^{\prime}\right), & i=1, \ldots, p, \\ w_{p+i}=\overline{E_{i}}\left(w^{\prime}, z^{\prime}\right), & i=1, \ldots, p .\end{cases}
$$

It is a complex submanifold of complex dimension $2 p$ with coordinates $\left(z^{\prime}, w^{\prime}\right) \in \mathbf{C}^{2 p}$. Let $\pi_{1}, \pi_{2}$ be the restrictions of the projections $(z, w) \rightarrow z$ and $(z, w) \rightarrow w$ to $\mathcal{M}$, respectively. Note that $\pi_{2}=C \pi_{1} \rho_{0}$, where $\rho_{0}$ is the restriction to $\mathcal{M}$ of the anti-holomorphic involution $(z, w) \rightarrow(\bar{w}, \bar{z})$ and $C$ is the complex conjugate.

Our basic assumption is the following condition.

Condition B. $q\left(z^{\prime}\right)=\left(q_{1}\left(z^{\prime}\right), \ldots, q_{p}\left(z^{\prime}\right)\right)$ satisfies $q^{-1}(0)=\{0\}$. 
When $p=1$, condition B corresponds to the non-vanishing of the Bishop invariant $\gamma$. When $\gamma=0$, Moser [Mos85] obtained a formal normal form that is still subject to further formal changes of coordinates. In [HY09a, Huang and Yin obtained a formal normal form and a complete holomorphic classification for real analytic surfaces with $\gamma=0$. The formal normal forms for co-dimension two real submanifolds in $\mathbf{C}^{n}$ have been further studied by Huang-Yin [HY12] and Burcea [Bur13]. Coffman [Cof06] showed that any $m$ dimensional real analytic submanifold in $\mathbf{C}^{n}$ of one-dimensional complex tangent space at a CR singularity satisfying certain non-degeneracy conditions is locally holomorphically equivalent to a unique algebraic submanifold, provided $2(n+1) / 3 \leq m<n$.

When $M$ is a quadric, i.e. all $E_{j}$ in (1.1) are quadratic polynomials, our basic condition B is equivalent to $\pi_{1}$ being a $2^{p}$-to- 1 branched covering. Since $\pi_{2}=C \pi_{1} \rho_{0}$, then $\pi_{2}$ is also a $2^{p}$-to- 1 branched covering. We will see that the $\mathrm{CR}$ singularities of the real submanifolds are closely connected with these branched coverings and their deck transformations.

We now introduce our main results. Some of them are analogous to the Moser-Wester theory. We will also describe new situations which arise with the maximum complex tangency.

1.3. Branched coverings and deck transformations. In section 2, we study the existence of deck transformations for $\pi_{1}$. We show that they must be involutions generating an abelian group of order $2^{k}$ for some $0 \leq k \leq p$. The latter is a major difference with the case $p=1$. Indeed, in the Moser-Webster theory, the group of deck transformations is generated by a unique non-trivial involution $\tau_{1}$. Therefore, we will impose the following condition.

Condition D. $M$ satisfies condition $B$ and the branched covering $\pi_{1}$ of $\mathcal{M}$ admits the maximum $2^{p}$ deck transformations.

Condition $\mathrm{D}$ gives rise to two families of commuting involutions $\left\{\tau_{i 1}, \ldots, \tau_{i 2^{p}}\right\}$ intertwined by the anti-holomorphic involution $\rho_{0}:\left(z^{\prime}, w^{\prime}\right) \rightarrow\left(\bar{w}^{\prime}, \bar{z}^{\prime}\right)$ such that $\tau_{2 j}=\rho_{0} \tau_{1 j} \rho_{0}(1 \leq$ $j \leq 2^{p}$ ) are deck transformations of $\pi_{2}$. We will call $\left\{\tau_{11}, \ldots, \tau_{12^{p}}, \rho_{0}\right\}$ the set of MoserWebster involutions. We will show that there is a unique set of $p$ generators for the deck transformations of $\pi_{1}$, denoted by $\tau_{11}, \ldots, \tau_{1 p}$, such that each $\tau_{1 j}$ fixes a hypersurface in $\mathcal{M}$ pointwise. Then

$$
\tau_{1}=\tau_{11} \circ \cdots \circ \tau_{1 p}
$$

is the unique deck transformation of which the fixed-point set has the smallest dimension $p$. Let $\tau_{2}=\rho_{0} \tau_{1} \rho_{0}$ and

$$
\sigma=\tau_{1} \tau_{2}
$$

Then $\sigma$ is reversible by $\tau_{j}$ and $\rho_{0}$, i.e. $\sigma^{-1}=\tau_{j} \sigma \tau_{j}^{-1}$ and $\sigma^{-1}=\rho_{0} \sigma \rho_{0}$.

As in the Moser-Webster theory, we will show that the existence of such $2^{p}$ deck transformations transfers the normal form problem for the real submanifolds into the normal form problem for the sets of involutions $\left\{\tau_{11}, \ldots, \tau_{1 p}, \rho_{0}\right\}$.

In this paper we will make the following assumption.

Condition J. $M$ satisfies condition $D$ and $M$ is diagonalizable, i.e. $\sigma^{\prime}(0)$ is diagonalizable.

Note that the condition excludes the higher dimensional analogous complex tangency of parabolic type, i.e. of $\gamma=1 / 2$. The normal form problem for the parabolic complex 
tangents has been studied by Webster [Web92, and in Gon96] where the normalization is divergent in general. In [AG09, Ahern and Gong constructed a moduli space for real analytic submanifolds that are formally equivalent to the Bishop quadric with $\gamma=1 / 2$.

1.4. Product quadrics. In this paper, the basic model for quadric manifolds with a CR singularity satisfying condition $\mathrm{J}$ is a product of 3 types of quadrics defined by

$$
\begin{gathered}
Q_{\gamma_{e}} \subset \mathbf{C}^{2}: z_{2}=\left(z_{1}+2 \gamma_{e} \bar{z}_{1}\right)^{2} ; \\
Q_{\gamma_{h}} \subset \mathbf{C}^{2}: z_{2}=\left(z_{1}+2 \gamma_{h} \bar{z}_{1}\right)^{2}, 1 / 2<\gamma_{h}<\infty ; \quad Q_{\infty}: z_{2}=z_{1}^{2}+\bar{z}_{1}^{2} ; \\
Q_{\gamma_{s}} \subset \mathbf{C}^{4}: z_{3}=\left(z_{1}+2 \gamma_{s} \bar{z}_{2}\right)^{2}, \quad z_{4}=\left(z_{2}+2\left(1-\bar{\gamma}_{s}\right) \bar{z}_{1}\right)^{2} .
\end{gathered}
$$

Here $\gamma_{s} \in \mathbf{C}$ and

$$
0<\gamma_{e}<1 / 2, \quad 1 / 2<\gamma_{h} \leq \infty, \quad \operatorname{Re} \gamma_{s} \leq 1 / 2, \quad \operatorname{Im} \gamma_{s} \geq 0, \quad \gamma_{s} \neq 0,1 / 2 .
$$

Note that $Q_{\gamma_{e}}, Q_{\gamma_{h}}$ are elliptic and hyperbolic Bishop quadrics, respectively. Realizing a type of pairs of involutions introduced in [Sto07], we will say that the complex tangent of $Q_{\gamma_{s}}$ at the origin is complex. We emphasize that this last type of quadric is new as it is not holomorphically equivalent to a product of two Bishop surfaces. A product of the above quadrics will be called a product of quadrics, or a product quadric. We denote by $e_{*}, h_{*}, 2 s_{*}$ the number of elliptic, hyperbolic and complex coordinates, respectively. We remark that the complex tangent of complex type has another basic model $Q_{\gamma_{s}}$ with $\gamma_{s}=1 / 2$, which is excluded by condition $\mathrm{J}$ (see Proposition 2.10).

This is the first part of two papers devoted to the local study of real analytic manifold at maximal complex tangent point. To limit its scope, we have to leave the complete classification of quadratic submanifolds of maximum deck transformations to the second paper [GS15] (see Theorem 1.1 therein), showing that there are quadratic manifolds which are not holomorphically equivalent to a product quadric. In [GS15], we also show that all Poincaré-Dulac normal forms of the $\sigma$ of a general higher order perturbation of a product quadric are divergent when $p>1$. With the divergent Poincaré-Dulac normal forms at our disposal, we seek types of CR singularities that ensure the convergent normalization and the analytic structure of the hull of holomorphy associated with the types of CR singularities.

We now introduce our main geometrical and dynamical results for analytic higher order perturbations of product quadric. We first turn to a holomorphic normalization of a real analytic submanifold $M$ with the so-called abelian CR singularity. This will be achieved by studying an integrability problem on a general family of commuting biholomorphisms described below. The holomorphic normalization will be used to construct the local hull of holomorphy of $M$. We will also study the rigidity problem of a quadric under higher order analytic perturbations, i.e. the problem if such a perturbation remains holomorphically equivalent to the quadric if it is formally equivalent to the quadric. The rigidity problem is reduced to a theorem of holomorphic linearization of one or several commuting diffeomorphisms that was devised in [Sto15]. Finally, we will study the existence of holomorphic submanifolds attached to the real submanifold $M$. These are complex submanifolds of dimension $p$ intersecting $M$ along two totally real analytic submanifolds that intersect transversally at a CR singularity. Attaching complex submanifolds has less constraints 
than finding a convergent normalization. A remarkable feature of attached complex submanifolds is that their existence depends only on the existence of suitable (convergent) invariant submanifolds of $\sigma$.

\subsection{Normal form of commuting biholomorphisms.}

Definition 1.1. Let $\mathcal{F}=\left\{F_{1}, \ldots, F_{\ell}\right\}$ be a finite family of germs of biholomorphisms of $\mathbf{C}^{n}$ fixing the origin. Let $D_{m}$ be the linear part of $F_{m}$ at the origin. We say that the family $\mathcal{F}$ is (resp. formally) completely integrable, if there is a (resp. formal) biholomorphic mapping $\Phi$ such that $\left\{\Phi^{-1} F_{m} \Phi: 1 \leq m \leq \ell\right\}=\left\{\hat{F}_{m}: 1 \leq m \leq \ell\right\}$ satisfies

(i) $\hat{F}_{m}(z)=\left(\mu_{m 1}(z) z_{1}, \ldots, \mu_{m n}(z) z_{n}\right)$ where $\mu_{m j}$ are germs of holomorphic (resp. formal) functions such that $\mu_{m j} \circ D_{m^{\prime}}=\mu_{m j}$ for $1 \leq m, m^{\prime} \leq \ell$ and $1 \leq j \leq n$. In particular, $\hat{F}_{m}$ commutes with $D_{m^{\prime}}$ for all $1 \leq m, m^{\prime} \leq \ell$.

(ii) For each $j$ and each $Q \in \mathbf{N}^{n}$ with $|Q|>1, \mu_{m}^{Q}(0)=\mu_{m j}(0)$ hold for all $m$ if and only if $\mu_{m}^{Q}(z)=\mu_{m j}(z)$ hold for all $m$.

A necessary condition for $\mathcal{F}$ to be formally completely integrable is that $F_{1}, \ldots, F_{\ell}$ commute pairwise. The main result of section 4 is the following.

Theorem 1.2. Let $\mathcal{F}$ be a family of finitely many germs of biholomorphisms at the origin. If $\mathcal{F}$ is formally completely integrable and its linear part $\mathcal{D}$ has the Poincaré type, then it is holomorphically completely integrable.

The definition of Poincaré type is in Definition 4.11. Such a formal integrability condition can hold under some geometrical properties. For instance, for a single germ of real analytic hyperbolic area-preserving mapping, the result was due to Moser [Mos56], and for a single germ of reversible hyperbolic holomorphic mapping $\sigma=\tau_{1} \tau_{2}$ of which $\tau_{1}$ fixes a hypersurface, this result was due to Moser-Webster [MW83]. Such results for commuting germs of vector fields were obtained in [Sto00, Sto05] under a collective small divisors Brjuno-type condition. Our result is inspired by these results.

1.6. Holomorphic normalization for the abelian CR singularity. In section 5, we obtain the convergent normalization for an abelian $\mathrm{CR}$ singularity which we now define. We first consider a product quadric $Q$ which satisfies condition J. So the deck transformations of $\pi_{1}$ for the complexification of $Q$ are generated by $p$ involutions of which each fixes a hypersurface pointwise. We denote them by $T_{11}, \ldots, T_{1 p}$. Let $T_{2 j}=\rho T_{1 j} \rho$. It turns out that each $T_{1 j}$ commutes with all $T_{i k}$ except one, $T_{2 k_{j}}$ for some $1 \leq k_{j} \leq p$. When we formulate $S_{j}=T_{1 j} T_{2 k_{j}}$ for $1 \leq j \leq p$, the $S_{1}, \ldots, S_{p}$ commute pairwise. Consider a general $M$ that is a third-order perturbation of product quadric $Q$ and satisfies condition J. We define $\sigma_{j}=\tau_{1 j} \tau_{2 k_{j}}$. In suitable coordinates, $T_{i j}$ (resp. $S_{j}$ ) is the linear part of $\tau_{i j}$ (resp. $\sigma_{j}$ ) at the origin. We say that the complex tangent of a third order perturbation $M$ of a product quadric at the origin is of abelian type, if $\sigma_{1}, \ldots, \sigma_{p}$ commute pairwise. If each linear part $S_{j}$ of $\sigma_{j}$ has exactly two eigenvalues $\mu_{j}, \mu_{j}^{-1}$ that are different from 1 , then $\mathcal{S}:=\left\{S_{1}, \ldots, S_{p}\right\}$ is of Poincaré type if and only if $\left|\mu_{j}\right| \neq 1$ for all $j$. As mentioned previously, Moser and Webster actually dealt with $n$-dimensional real submanifolds in $\mathbf{C}^{n}$ that have the minimal dimension of complex tangent subspace at a CR singular point. In their situation, there is only one possible composition, that is $\sigma=\tau_{1} \tau_{2}$. When the complex tangent has an 
elliptic but non-vanishing Bishop invariant, $\sigma$ has exactly two positive eigenvalues that are separated by 1 , while the remaining eigenvalues are 1 with multiplicity $n-2$.

As an application of Theorem 1.2, we will prove the following convergent normalization.

Theorem 1.3. Let $M$ be a germ of real analytic submanifold in $\mathbf{C}^{2 p}$ that is a third order perturbation of a product quadric given by (1.2)-(1.5) with an abelian CR singularity. Suppose that $M$ has all eigenvalues of modulus different from one, i.e. it has no hyperbolic component $\left(h_{*}=0\right)$ while each $\gamma_{s}$ in (1.4) satisfies $\operatorname{Re} \gamma_{s}<1 / 2$ additionally. Then $M$ is holomorphically equivalent to

$$
\widehat{M}: z_{p+j}=\Lambda_{1 j}(\zeta) \zeta_{j}, \quad \Lambda_{1 j}(0)=\lambda_{j}, \quad 1 \leq j \leq p,
$$

where $\zeta=\left(\zeta_{1}, \ldots, \zeta_{p}\right)$ are the solutions to

$$
\begin{aligned}
\zeta_{e} & =A_{e}(\zeta) z_{e} \bar{z}_{e}-B_{e}(\zeta)\left(z_{e}^{2}+\bar{z}_{e}^{2}\right), \quad 1 \leq e \leq e_{*}, \\
\zeta_{s} & =A_{s}(\zeta) z_{s} \bar{z}_{s+s_{*}}-B_{s}(\zeta)\left(z_{s}^{2}+\Lambda_{1 s}^{2}(\zeta) \bar{z}_{s+s_{*}}^{2}\right), \quad e_{*}<s \leq e_{*}+s_{*}, \\
\zeta_{s+s_{*}} & =A_{s+s_{*}}(\zeta) \bar{z}_{s} z_{s+s_{*}}-B_{s+s_{*}}(\zeta)\left(z_{s+s_{*}}^{2}+\Lambda_{1\left(s+s_{*}\right)}^{2}(\zeta) \bar{z}_{s}^{2}\right),
\end{aligned}
$$

while $\Lambda_{1 j}$ satisfies (5.6)-(5.7), and $A_{j}, B_{j}$ are rational functions in $\Lambda_{1 j}$ defined by (5.16)(5.17).

There are many non-product real submanifolds of abelian CR singularity.

Example 1.4. Let $0<\gamma_{i}<\infty$. Let $R\left(z_{1}, \bar{z}_{1}\right)=\left|z_{1}\right|^{2}+\gamma_{1}\left(z_{1}^{2}+\bar{z}_{1}^{2}\right)+O(3)$ be a real-valued power series in $z_{1}, \bar{z}_{1}$ of real coefficients. Then the origin is an abelian CR singularity of

$$
M: z_{3}=R\left(z_{1}, \bar{z}_{1}\right), \quad z_{4}=\left(z_{2}+2 \gamma_{2} \bar{z}_{2}+z_{2} z_{3}\right)^{2} .
$$

We will also present a more direct proof of Theorem 1.3 by using a convergence theorem of Moser and Webster [MW83] and some formal results from section 4. The above $\Lambda_{11}, \ldots, \Lambda_{1 p}$ satisfy conditions $\Lambda_{1 j}(0)=\lambda_{j}$ and (5.6)-(5.7) and are otherwise arbitrary convergent power series. The $\Lambda_{11}, \ldots, \Lambda_{1 p}$ may be subjected to further normalization. In [GS15], we find a unique holomorphic normal form by refining the above normalization for $M$ satisfying a non resonance condition and a third order non-degeneracy condition (see Theorem 5.6 in [GS15]); in particular, it shows the existence of infinitely many formal invariants and non-product structures of the manifolds when $p>1$.

As an application of Theorem 1.3, we will prove the following flattening result.

Corollary 1.5. Let $M$ be as in Theorem 1.3. In suitable holomorphic coordinates, $M$ is contained in the linear subspace defined by $z_{p+e}=\bar{z}_{p+e}$ and $z_{p+s}=\bar{z}_{p+s+s_{*}}$ where $1 \leq e \leq e_{*}$ and $e_{*}<s \leq e_{*}+s_{*}$.

1.7. Analytic hull of holomorphy. One of significances of the Bishop quadrics is that their higher order analytic perturbation at an elliptic complex tangent has a non-trivial hull of holomorphy. As another application of the above normal form, we will construct the local hull of holomorphy of $M$ via higher dimensional non-linear analytic polydiscs.

Corollary 1.6. Let $M$ be as in Theorem 1.3. Suppose that $M$ has only elliptic component of complex tangent. Then in suitable holomorphic coordinates, $\mathcal{H}_{\text {loc }}(M)$, the local hull of holomorphy of $M$, is filled by a real analytic family of analytic polydiscs of dimension $p$. 
For a precise statement of the corollary, see Theorem 5.5. The hulls of holomorphy for real submanifolds with a CR singularity have been studied extensively, starting with the work of Bishop. In the real analytic case with minimum complex tangent space at an elliptic complex tangent, we refer to Moser-Webster [MW83] for $\gamma>0$, and Krantz-Huang [HK95] for $\gamma=0$. For the smooth case, see Kenig-Webster [KW82, KW84], Huang [Hua98]. For global results on hull of holomorphy, we refer to [BG83, BK91].

\subsection{Rigidity of quadrics. In Section 6, we prove the following theorem.}

Theorem 1.7. Let $M$ be a germ of analytic submanifold that is an higher order perturbation of a product quadric $Q$ in $\mathbf{C}^{2 p}$ given by (1.2)-(1.5). Assume that $M$ is formally equivalent to $Q$. Suppose that each hyperbolic component has an eigenvalue $\mu_{h}$ which is either a root of unity or satisfies Brjuno condition, and each complex component has an eigenvalue $\mu_{s}$ is not a root of unity and satisfies the Brjuno condition. Then $M$ is holomorphically equivalent to the product quadric.

We emphasize that condition (1.5) ensure that $M$ is diagonalizable (condition $\mathrm{J}$ ). It is plausible that theorem remains valid when $M$ satisfies condition $\mathrm{J}$ and $\mu_{s}$ satisfy the Brjuno condition or are roots of unity; however, the resonance condition requires some tedious changes of computation in section 6 . The proof uses a theorem of linearization of holomorphic mappings in [Sto15]. Brjuno small divisors condition is defined by (7.37), with $\nu=\mu_{h}$ and $p=1$. When $p=1$, the result under the stronger Siegel condition is in Gon94. This last statement requires a small divisors condition to be true as shown in Gon04. When $p=1$ with a vanishing Bishop invariant, such rigidity result was obtained by Moser [Mos85] and by Huang-Yin [HY09b] in a more general context.

1.9. Attached complex submanifolds. We now describe convergent results for attached complex submanifolds. The results are for a general $M$, including the one of which the complex tangent might not be of abelian type.

We say that a formal complex submanifold $K$ is attached to $M$ if $K \cap M$ contains at least two germs of totally real and formal submanifolds $K_{1}, K_{2}$ that intersect transversally at a given CR singularity. In [Kli85], Klingenberg showed that when $M$ is non-resonant and $p=1$, there is a unique formal holomorphic curve attached to $M$ with a hyperbolic complex tangent. He also proved the convergence of the attached formal holomorphic curve under a Siegel small divisors condition. When $p>1$, we will show that generically there is no formal complex submanifold that can be attached to $M$ if the CR singularity has an elliptic component. When $p>1$ and $M$ is a higher order perturbation of a product quadric of $Q_{\gamma_{h}}, Q_{\gamma_{s}}$, we will encounter various interesting situations.

By adapting Klingenberg's proof for $p=1$ and using a theorem of Pöschel [Pös86], we will prove the following.

Theorem 1.8. Let $M$ be a germ of analytic submanifold that is an higher order perturbation of a product quadric $Q$ in $\mathbf{C}^{2 p}$ without elliptic components. Assume that the eigenvalues $\mu_{1}, \ldots, \mu_{p}, \mu_{1}^{-1}, \ldots, \mu_{p}^{-1}$ of $D \sigma(0)$ are distinct. Let $\epsilon_{h}^{2}, \epsilon_{s}^{2}=1, \nu_{h}:=\mu_{h}^{\epsilon_{h}}, \nu_{s}:=\bar{\mu}_{s}^{\epsilon_{s}}$ and $\nu_{s+s_{*}}:=\bar{\nu}_{s}^{-1}$. Assume $\nu=\left(\nu_{1}, \ldots, \nu_{p}\right)$ is weakly non resonant and Diophantine in the sense of Pöschel. Then $M$ admits an attached complex submanifold $M_{\epsilon}$. 
Weak non resonance is defined in (7.34), while Diophantine condition in the sense of Pöschel is defined in (7.37).

Finally, we prove the convergence of all attached formal submanifolds:

Theorem 1.9. Let $M$ be as in Theorem 1.8. Suppose that the $2 p$ eigenvalues of $\sigma$ are non-resonant. If the eigenvalues of $\sigma$ satisfy a Bruno type condition, all attached formal submanifolds are convergent.

The Brjuno-type condition, defined in (7.38), was introduced in Sto15] for linearization on ideals.

1.10. Notation. We denote the identity map by $I$ and by $L F$ the linear part at the origin of a mapping $F:\left(\mathbf{C}^{m}, 0\right) \rightarrow\left(\mathbf{C}^{n}, 0\right)$. We also denote by $D F(z)$ or $F^{\prime}(z)$, the Jacobian matrix of $F$ at $z$. By an analytic (or holomorphic) function, we mean a germ of analytic function at a point (which will be defined by the context) otherwise stated. We denote by $\mathcal{O}_{n}$ (resp. $\widehat{\mathcal{O}}_{n}, \mathfrak{M}_{n}, \widehat{\mathfrak{M}}_{n}$ ) the space of germs of holomorphic functions of $\mathbf{C}^{n}$ at the origin (resp. of formal power series in $\mathbf{C}^{n}$, holomorphic germs, and formal germs vanishing at the origin). If $Q=\left(q_{1}, \ldots, q_{k}\right) \in \mathbf{N}^{k}$, then $|Q|=q_{1}+\cdots+q_{k}$ and $x^{Q}=x_{1}^{q_{1}} \cdots x_{k}^{q_{k}}$.

Acknowledgment. This joint work was completed while X.G. was visiting at SRC-GAIA of POSTECH. He is grateful to Kang-Tae Kim for hospitality.

\section{CR SINGULARITIES AND DECK TRANSFORMATIONS}

We consider a real submanifold $M$ of $\mathbf{C}^{n}$. Let $T_{x_{0}}^{(1,0)} M$ be the space of tangent vectors of $M$ at $x_{0}$ of the form $\sum_{j=1}^{n} a_{j} \frac{\partial}{\partial z_{j}}$. Let $M$ have dimension $n$. In this paper, we assume that $T_{x_{0}}^{(1,0)} M$ has the largest possible dimension $p=n / 2$ at a given point $x_{0}$. In suitable holomorphic affine coordinates, we have $x_{0}=0$ and

$$
M: z_{p+j}=E_{j}\left(z^{\prime}, \bar{z}^{\prime}\right), \quad 1 \leq j \leq p .
$$

Here we set $z^{\prime}=\left(z_{1}, \ldots, z_{p}\right)$ and we will denote $z^{\prime \prime}=\left(z_{p+1}, \ldots, z_{2 p}\right)$. Also, the $E_{j}$ together with their first order derivatives vanish at 0 . The tangent space $T_{0} M$ is then the $z^{\prime}$-subspace.

The main purpose of this section is to obtain some basic invariants and a relation between two families of involutions and the real analytic submanifolds which we want to normalize.

Note that $M$ is totally real at $\left(z^{\prime}, z^{\prime \prime}\right) \in M$ if and only if $C\left(z^{\prime}, \bar{z}^{\prime}\right) \neq 0$, where $C\left(z^{\prime}, \overline{z^{\prime}}\right):=$ $\operatorname{det}\left(\frac{\partial E_{i}}{\partial \bar{z}_{j}}\right)_{1 \leq i, j \leq p}$. We will assume that $C\left(z^{\prime}, \bar{z}^{\prime}\right)$ is not identically zero in any neighborhood of the origin. Then the zero set of $C$ on $M$, denoted by $M_{C R \text { sing }}$, is called $C R$ singular set of $M$, or the set of complex tangents of $M$. We assume that $M$ is real analytic. Then $M_{C R s i n g}$ is a possibly singular proper real analytic subset of $M$ that contains the origin.

2.1. Existence of deck transformations and examples. We first derive some quadratic invariants. Applying a quadratic change of holomorphic coordinates, we obtain

$$
E_{j}\left(z^{\prime}, \bar{z}^{\prime}\right)=h_{j}\left(z^{\prime}, \bar{z}^{\prime}\right)+q_{j}\left(\bar{z}^{\prime}\right)+O\left(\left|\left(z^{\prime}, \bar{z}^{\prime}\right)\right|^{3}\right) .
$$

Here we have used the convention that if $x=\left(x_{1}, \ldots, x_{n}\right)$, then $O\left(|x|^{k}\right)$ denotes a formal power series in $x$ without terms of order $<k$. A biholomorphic map $f$ that preserves the form of the above submanifolds $M$ and fixes the origin must preserve their complex tangent 
spaces at the origin, i.e. $z^{\prime \prime}=0$. Thus if $\tilde{z}$ denote the old coordinates and $z$ denote the new coordinates then $f$ has the form

$$
\tilde{z}^{\prime}=\mathbf{A} z^{\prime}+\mathbf{B} z^{\prime \prime}+O\left(|z|^{2}\right), \quad \tilde{z}^{\prime \prime}=\mathbf{U} z^{\prime \prime}+O\left(|z|^{2}\right) .
$$

Here $\mathbf{A}$ and $\mathbf{U}$ are non-singular $p \times p$ complex matrices. Now $f(M)$ is given by

$$
\mathbf{U} z^{\prime \prime}=h\left(\mathbf{A} z^{\prime}, \overline{\mathbf{A}} \bar{z}^{\prime}\right)+q\left(\overline{\mathbf{A}} \bar{z}^{\prime}\right)+O\left(|z|^{3}\right) .
$$

We multiply the both sides by $\mathbf{U}^{-1}$ and solve for $z^{\prime \prime}$; the vectors of $p$ quadratic forms $\left\{h\left(\tilde{z}^{\prime}, \overline{\tilde{z}^{\prime}}\right), q\left(\tilde{z}^{\prime}\right)\right\}$ are transformed into

$$
\left\{\hat{h}\left(z^{\prime}, z^{\prime}\right), \hat{q}\left(\bar{z}^{\prime}\right)\right\}=\left\{\mathbf{U}^{-1} h\left(\mathbf{A} z^{\prime}, \overline{\mathbf{A}} \bar{z}^{\prime}\right), \mathbf{U}^{-1} q\left(\overline{\mathbf{A}} \bar{z}^{\prime}\right)\right\} .
$$

This shows that if $M$ and $\hat{M}$ are holomorphically equivalent, their corresponding quadratic terms are equivalent via (2.3). Therefore, we obtain a holomorphic invariant

$$
q_{*}=\operatorname{dim}_{\mathbf{C}}\left\{z^{\prime}: q_{1}\left(z^{\prime}\right)=\cdots=q_{p}\left(z^{\prime}\right)=0\right\} .
$$

We remark that when $M, \hat{M}$ are quadratic (i.e. when their corresponding $E, \hat{E}$ are homogeneous quadratic polynomials), the equivalence relation (2.3) implies that $M, \hat{M}$ are linearly equivalent, Therefore, the above transformation of $h$ and $q$ via $\mathbf{A}$ and $\mathbf{U}$ determines the classifications of the quadrics under local biholomorphisms as wells as under linear biholomorphisms. We have shown that the two classifications for the quadrics are identical.

Recall that $M$ is real analytic. Let us complexify such a real submanifold $M$ by replacing $\bar{z}^{\prime}$ by $w^{\prime}$ to obtain a complex $n$-submanifold of $\mathbf{C}^{2 n}$, defined by

$$
\mathcal{M}:\left\{\begin{array}{l}
z_{p+i}=E_{i}\left(z^{\prime}, w^{\prime}\right), \\
w_{p+i}=\bar{E}_{i}\left(w^{\prime}, z^{\prime}\right), \quad i=1, \ldots, p .
\end{array}\right.
$$

We use $\left(z^{\prime}, w^{\prime}\right)$ as holomorphic coordinates of $\mathcal{M}$ and define the anti-holomorphic involution $\rho$ on it by

$$
\rho\left(z^{\prime}, w^{\prime}\right)=\left(\bar{w}^{\prime}, \bar{z}^{\prime}\right) .
$$

Occasionally we will also denote the above $\rho$ by $\rho_{0}$ for clarity. We will identify $M$ with a totally real and real analytic submanifold of $\mathcal{M}$ via embedding $z \rightarrow(z, \bar{z})$. We have $M=\mathcal{M} \cap \operatorname{Fix}(\rho)$ where $\operatorname{Fix}(\rho)$ denotes the set of fixed points of $\rho$. Let $\pi_{1}: \mathcal{M} \mapsto \mathbf{C}^{n}$ be the restriction of the projection $(z, w) \rightarrow z$ and let $\pi_{2}$ be the restriction of $(z, w) \rightarrow w$. It is clear that $\pi_{2}=\overline{\pi_{1} \rho}$ on $\mathcal{M}$. Throughout the paper, $\pi_{1}, \pi_{2}, \rho$ are restricted on $\mathcal{M}$ unless stated otherwise.

Condition B that $q_{*}=0$, introduced in section 1, ensures that $\pi_{1}$ is a branched covering. A necessary condition for $q_{*}=0$ is that functions $q_{1}\left(z^{\prime}\right), q_{2}\left(z^{\prime}\right), \ldots, q_{p}\left(z^{\prime}\right)$ are linearly independent, since the intersection of $k$ germs of holomorphic hypersurfaces at 0 in $\mathbf{C}^{p}$ has dimension at least $p-k$. (See [Chi89], p. 35; Gun90][Corollary 8, p. 81].)

When $\pi_{1}: \mathcal{M} \rightarrow \mathbf{C}^{2 p}$ is a branched covering, we define a deck transformation on $\mathcal{M}$ for $\pi_{1}$ to be a germ of biholomorphic mapping $F$ defined at $0 \in \mathcal{M}$ that satisfies $\pi_{1} \circ F=\pi_{1}$. In other words, $F\left(z^{\prime}, w^{\prime}\right)=\left(z^{\prime}, f\left(z^{\prime}, w^{\prime}\right)\right)$ and

$$
E_{i}\left(z^{\prime}, w^{\prime}\right)=E_{i}\left(z^{\prime}, f\left(z^{\prime}, w^{\prime}\right)\right), \quad i=1, \ldots, p .
$$


Lemma 2.1. Suppose that $q_{*}=0$. Then $M_{C R \operatorname{sing}}$ is a proper real analytic subset of $M$ and $M$ is totally real away from $M_{C R s i n g}$, i.e. the $C R$ dimension of $M$ is zero. Furthermore, $\pi_{1}$ is a $2^{p}$-to-1 branched covering. The group of deck transformations of $\pi_{1}$ consists of $2^{\ell}$ commuting involutions with $0 \leq \ell \leq p$.

Proof. Since $q^{-1}(0)=\{0\}$, then $z^{\prime} \rightarrow q\left(z^{\prime}\right)$ is a finite holomorphic map; see [Chi89], p. 105 . Hence its Jacobian determinant is not identically zero. In particular, $\operatorname{det}\left(\frac{\partial E_{i}}{\partial \bar{z}_{j}}\right)_{1 \leq i, j \leq p}$ is not identically zero. This shows that $M$ has CR dimension 0.

Since $w^{\prime} \rightarrow q\left(w^{\prime}\right)$ is a homogeneous quadratic mapping of the same space which vanishes only at the origin, then

$$
\left|q\left(w^{\prime}\right)\right| \geq c\left|w^{\prime}\right|^{2}
$$

We want to verify that $\pi_{1}$ is a $2^{p}$-to-1 branched covering. Let $\Delta_{r}=\{z \in \mathbf{C}:|z|<r\}$. We choose $C>0$ such that $\pi_{1}(z, w)=\left(z^{\prime}, E\left(z^{\prime}, w^{\prime}\right)\right)$ defines a proper and onto mapping

$$
\pi_{1}: \mathcal{M}_{1}:=\mathcal{M} \cap\left(\left(\Delta_{\delta}^{p} \times \Delta_{\delta^{2}}^{p}\right) \times\left(\Delta_{C \delta}^{p} \times \Delta_{C \delta^{2}}^{p}\right)\right) \mapsto \Delta_{\delta}^{p} \times \Delta_{\delta^{2}}^{p}
$$

By Sard's theorem, the regular values of $\pi_{1}$ have the full measure. For each regular value $z, \pi_{1}^{-1}(z)$ has exactly $2^{p}$ distinct points (see [Chi89], p. 105 and p. 112). It is obvious that $\mathcal{M}_{1}$ is smooth and connected. We fix a fiber $F_{z}$ of $2^{p}$ points. Then the group of deck transformations of $\pi_{1}$ acts on $F_{z}$ in such a way that if a deck transformation fixes a point in $F_{z}$, then it must be the identity. Therefore, the number of deck transformations divides $2^{p}$ and each deck transformation has period $2^{\ell}$ with $0 \leq \ell \leq p$.

We first show that each deck transformation $f$ of $\pi_{1}$ is an involution. We know that $f$ is periodic and has the form

$$
z^{\prime} \rightarrow z^{\prime}, \quad w^{\prime} \rightarrow \mathbf{A} w^{\prime}+\mathbf{B} z^{\prime}+O(2)
$$

where $\mathbf{A}, \mathbf{B}$ are matrices. Assume that $f$ has period $m$. Then $\hat{f}\left(z^{\prime}, w^{\prime}\right)=\left(z^{\prime}, \mathbf{A} w^{\prime}+\mathbf{B} z^{\prime}\right)$ satisfies $\hat{f}^{m}=I$ and $f$ is locally equivalent to $\hat{f}$; indeed $\hat{f} g f^{-1}=g$ for

$$
g=\sum_{i=1}^{m}\left(\hat{f}^{i}\right)^{-1} \circ f^{i} .
$$

Therefore, it suffices to show that $\hat{f}$ is an involution. We have

$$
\hat{f}^{m}\left(z^{\prime}, w^{\prime}\right)=\left(z^{\prime}, \mathbf{A}^{m} w^{\prime}+\left(\mathbf{A}^{m-1}+\cdots+\mathbf{A}+\mathbf{I}\right) \mathbf{B} z^{\prime}\right) .
$$

Since $f$ is a deck transformation, then $E\left(z^{\prime}, w^{\prime}\right)$ is invariant under $f$. Recall from (2.2) that $E\left(z^{\prime}, \bar{z}^{\prime}\right)$ starts with quadratic terms of the form $h\left(z^{\prime}, \bar{z}^{\prime}\right)+q\left(\bar{z}^{\prime}\right)$. Comparing quadratic terms in $E\left(z^{\prime}, w^{\prime}\right)=E \circ \hat{f}\left(z^{\prime}, w^{\prime}\right)$, we see that the linear map $\hat{f}$ has invariant functions

$$
z^{\prime \prime}=h\left(z^{\prime}, w^{\prime}\right)+q\left(w^{\prime}\right) .
$$

We know that $\mathbf{A}^{m}=\mathbf{I}$. By the Jordan normal form, we choose a linear transformation $\tilde{w}^{\prime}=\mathbf{S} w^{\prime}$ such that $\mathbf{S A} \mathbf{S}^{-1}$ is the diagonal matrix $\operatorname{diag} \mathbf{a}$ with $\mathbf{a}=\left(a_{1}, \ldots, a_{p}\right)$. In $\left(z^{\prime}, \tilde{w}^{\prime}\right)$ coordinates, the mapping $\hat{f}$ has the form $\left(z^{\prime}, \tilde{w}^{\prime}\right) \rightarrow\left(z^{\prime},(\operatorname{diag} \mathbf{a}) \tilde{w}^{\prime}+\mathbf{S B} z^{\prime}\right)$. Now

$$
\tilde{h}_{j}\left(z^{\prime}, \tilde{w}^{\prime}\right)+\tilde{q}_{j}\left(\tilde{w}^{\prime}\right):=h_{j}\left(z^{\prime}, \mathbf{S}^{-1} \tilde{w}\right)+q_{j}\left(\mathbf{S}^{-1} \tilde{w}^{\prime}\right)
$$


are invariant under $\hat{f}$. Hence $\tilde{q}_{j}\left(\tilde{w}^{\prime}\right)$ are invariant under $\tilde{w}^{\prime} \mapsto(\operatorname{diag} \mathbf{a}) \tilde{w}^{\prime}$. Since the common zero set of $q_{1}\left(w^{\prime}\right), \ldots, q_{p}\left(w^{\prime}\right)$ is the origin, then

$$
V=\left\{\tilde{w}^{\prime} \in \mathbf{C}^{p}: \tilde{q}\left(\tilde{w}^{\prime}\right)=0\right\}=\{0\} .
$$

We conclude that $\tilde{q}\left(\tilde{w}_{1}, 0, \ldots, 0\right)$ is not identically zero; otherwise $V$ would contain the $\tilde{w}_{1}$-axis. Now $\tilde{q}\left((\operatorname{diag} \mathbf{a}) \tilde{w}^{\prime}\right)=\tilde{q}\left(\tilde{w}^{\prime}\right)$, restricted to $\tilde{w}^{\prime}=\left(\tilde{w}_{1}, 0, \ldots, 0\right)$, implies that $a_{1}= \pm 1$. By the same argument, we get $a_{j}= \pm 1$ for all $j$. This shows that $\mathbf{A}^{2}=\mathbf{I}$. Let us combine it with

$$
\mathbf{A}^{m}=\mathbf{I}, \quad\left(\mathbf{A}^{m-1}+\cdots+\mathbf{A}+\mathbf{I}\right) \mathbf{B}=\mathbf{0} .
$$

If $m=1$, it is obvious that $\hat{f}=I$. If $m=2^{\ell}>1$, then $(\mathbf{A}+\mathbf{I}) \mathbf{B}=\mathbf{0}$. Thus $\hat{f}^{2}\left(z^{\prime}, w^{\prime}\right)=$ $\left(z^{\prime}, \mathbf{A}^{2} w^{\prime}+(\mathbf{A}+\mathbf{I}) \mathbf{B} z^{\prime}\right)=\left(z^{\prime}, w^{\prime}\right)$. This shows that every deck transformation of $\pi_{1}$ is an involution.

For any two deck transformations $f$ and $g, f g$ is still a deck transformation. Hence $(f g)^{2}=I$ implies that $f g=g f$.

Before we proceed to discussing the deck transformations, we give some examples. The first example turns out to be a holomorphic equivalent form of a real submanifold that admits the maximum number of deck transformations and satisfies other mild conditions.

Example 2.2. Let $\mathbf{B}=\left(b_{j k}\right)$ be a non-singular $p \times p$ matrix. Let $M$ be defined by

$$
z_{p+j}=\left(\sum_{k} b_{j k} \bar{z}_{k}+R_{j}\left(z^{\prime}, \bar{z}^{\prime}\right)\right)^{2}, \quad 1 \leq j \leq p,
$$

where each $R_{j}\left(0, \bar{z}^{\prime}\right)$ starts with terms of order at least 2 . Then $M$ admits $2^{p}$ deck transformations for $\pi_{1}$. Indeed, let $\mathbf{E}_{1}, \ldots, \mathbf{E}_{2^{p}}$ be the set of diagonal $p \times p$ matrices with $\mathbf{E}_{j}^{2}=\mathbf{I}$, and let $\mathbf{R}$ be the column vector $\left(R_{1}, \ldots, R_{p}\right)^{t}$. For each $\mathbf{E}_{j}$ let us show that there is a deck transformation $\left(z^{\prime}, w^{\prime}\right) \rightarrow\left(z^{\prime}, \tilde{w}^{\prime}\right)$ satisfying

$$
\mathbf{B} \tilde{w}^{\prime}+\mathbf{R}\left(z^{\prime}, \tilde{w}^{\prime}\right)=\mathbf{E}_{j}\left(\mathbf{B} w^{\prime}+\mathbf{R}\left(z^{\prime}, w^{\prime}\right)\right) .
$$

Since $\mathbf{B}$ is invertible, it has a unique solution

$$
\tilde{w}^{\prime}=\mathbf{B}^{-1} \mathbf{E}_{j} \mathbf{B} w^{\prime}+O\left(\left|z^{\prime}\right|\right)+O\left(\left|w^{\prime}\right|^{2}\right) .
$$

Finally, $\left(z^{\prime}, w^{\prime}\right) \rightarrow\left(z^{\prime}, \tilde{w}^{\prime}\right)$ is an involution, as if $\left(z^{\prime}, w^{\prime}, \tilde{w}^{\prime}\right)=\left(z^{\prime}, w^{\prime}, f\left(z^{\prime}, w^{\prime}\right)\right)$ satisfy (2.7) if and only if $\left(z^{\prime}, f\left(z^{\prime}, w^{\prime}\right), w^{\prime}\right)$, substituting for $\left(z^{\prime}, w^{\prime}, \tilde{w}^{\prime}\right)$ in (2.7), satisfy (2.7).

We now present an example to show that the deck transformations can be destroyed by perturbations when $p>1$. This is the major difference between real submanifolds with $p>1$ and the ones with $p=1$. The example shows that the number of deck transformations can be reduced to any number $2^{\ell}$ by a higher order perturbation.

Example 2.3. Let $N_{\gamma, \epsilon}$ be a perturbation of $Q_{\gamma}$ defined by

$$
z_{p+j}=z_{j} \bar{z}_{j}+\gamma_{j} \bar{z}_{j}^{2}+\epsilon_{j-1} \bar{z}_{j-1}^{3}, \quad 1 \leq j \leq p
$$

Here $\epsilon_{j} \neq 0$ for all $j, \epsilon_{0}=\epsilon_{p}$ and $z_{0}=z_{p}$. Let $\tau$ be a deck transformation of $N_{\gamma, \epsilon}$ for $\pi_{1}$. We know that $\tau$ has the form

$$
z_{j}^{\prime}=z_{j}, \quad w_{j}^{\prime}=A_{j}\left(z^{\prime}, w^{\prime}\right)+B_{j}\left(z^{\prime}, w^{\prime}\right)+O\left(\left|\left(z^{\prime}, w^{\prime}\right)\right|^{3}\right) .
$$


Here $A_{j}$ are linear and $B_{j}$ are homogeneous quadratic polynomials. We then have

$$
\begin{gathered}
z_{j} A_{j}\left(z^{\prime}, w^{\prime}\right)+\gamma_{j} A_{j}^{2}\left(z^{\prime}, w^{\prime}\right)=z_{j} w_{j}+\gamma_{j} w_{j}^{2}, \\
z_{j} B_{j}\left(z^{\prime}, w^{\prime}\right)+2 \gamma_{j}\left(A_{j} B_{j}\right)\left(z^{\prime}, w^{\prime}\right)+\epsilon_{j-1} A_{j-1}^{3}\left(z^{\prime}, w^{\prime}\right)=\epsilon_{j-1} w_{j-1}^{3} .
\end{gathered}
$$

We know that $L \tau$ is a deck transformation for $Q_{\gamma}$. Thus $a_{j}\left(w^{\prime}\right):=A_{j}\left(0, w^{\prime}\right)= \pm w_{j}$. Set $z_{j}=0$ in (2.9) to get $a_{j}\left(w^{\prime}\right) \mid \epsilon_{j-1}\left(w_{j-1}^{3}-a_{j-1}^{3}\left(w^{\prime}\right)\right)$. Thus $a_{j-1}\left(w^{\prime}\right)=w_{j-1}$. Hence, the matrix of $L \tau$ is triangular and its diagonal entries are 1. Since $L \tau$ is periodic then $L \tau=I$. Since $\tau$ is periodic, then $\tau=I$.

Based the above example, we impose the basic condition $\mathrm{D}$ that the branched covering $\pi_{1}$ of $\mathcal{M}$ admits the maximum $2^{p}$ deck transformations.

We first derive some properties of real submanifolds under condition D.

2.2. Real submanifolds and Moser-Webster involutions. The main result of this subsection is to show the equivalence of classification of the real submanifolds satisfying condition $D$ with that of families of involutions $\left\{\tau_{11}, \ldots, \tau_{1 p}, \rho\right\}$. More precisely, condition $\mathrm{J}$ is not imposed. The relation between two classifications plays an important role in the Moser-Webster theory for $p=1$. This will be the base of our approach to the normal form problems.

Let $\mathcal{F}$ be a family of holomorphic maps in $\mathbf{C}^{n}$ with coordinates $z$. Let $L \mathcal{F}$ denote the set of linear maps $z \rightarrow f^{\prime}(0) z$ with $f \in \mathcal{F}$. Let $\mathcal{O}_{n}^{\mathcal{F}}$ denote the set of germs of holomorphic functions $h$ at $0 \in \mathbf{C}^{n}$ so that $h \circ f=h$ for each $f \in \mathcal{F}$. Let $\left[\mathfrak{M}_{n}\right]_{1}^{L \mathcal{F}}$ be the subset of linear functions of $\mathfrak{M}_{n}^{L \mathcal{F}}$.

Lemma 2.4. Let $G$ be an abelian group of holomorphic (resp. formal) involutions fixing $0 \in$ $\mathbf{C}^{n}$. Then $G$ has $2^{\ell}$ elements and they are simultaneously diagonalizable by a holomorphic (resp. formal) transformation. If $k=\operatorname{dim}_{\mathbf{C}}\left[\mathfrak{M}_{n}\right]_{1}^{L G}$ then $\ell \leq n-k$. Assume furthermore that $\ell=n-k$. In suitable holomorphic $\left(z_{1}, \ldots, z_{n}\right)$ coordinates, the group $G$ is generated by $Z_{k+1}, \ldots, Z_{n}$ with

$$
Z_{j}: z_{j}^{\prime}=-z_{j}, \quad z_{i}^{\prime}=z_{i}, \quad i \neq j, \quad 1 \leq i \leq n .
$$

In the $z$ coordinates, the set of convergent (resp. formal) power series in $z_{1}, \ldots, z_{k}$, $z_{k+1}^{2}, \ldots, z_{n}^{2}$ is equal to $\mathcal{O}_{n}^{G}\left(\right.$ resp. $\left.\widehat{\mathcal{O}}_{n}^{G}\right)$, and with $Z=Z_{n-k} \cdots Z_{n}$,

$$
\left[\mathfrak{M}_{n}\right]_{1}^{G}=\left[\mathfrak{M}_{n}\right]_{1}^{Z}, \quad \operatorname{Fix}(Z)=\bigcap_{j=k+1}^{n} \operatorname{Fix}\left(Z_{j}\right) .
$$

Proof. We first want to show that $G$ has $2^{\ell}$ elements. Suppose that it has more than one element and we have already found a subgroup of $G$ that has $2^{i}$ elements $f_{1}, \ldots, f_{2^{i}}$. Let $g$ be an element in $G$ that is different from the $2^{i}$ elements. Since $g$ is an involution and commutes with each $f_{j}$, then

$$
f_{1}, \ldots, f_{2^{i}}, \quad g f_{1}, \ldots, g f_{2^{i}}
$$

form a group of $2^{i+1}$ elements. We have proved that every finite subgroup of $G$ has exactly $2^{\ell}$ elements. Moreover, if $G$ is infinite then it contains a subgroup of $2^{\ell}$ elements for every $\ell \geq 0$. Let $\left\{f_{1}, \ldots, f_{2^{\ell}}\right\}$ be such a subgroup of $G$. It suffices to show that $\ell \leq n-k$. We first 
linearize all $f_{j}$ simultaneously. We know that $L f_{1}, \ldots, L f_{2^{\ell}}$ commute pairwise. Note that $I+f_{1}^{\prime}(0)^{-1} f_{1}$ linearizes $f_{1}$. Assume that $f_{1}$ is linear. Then $f_{1}=L f_{1}$ and $L f_{2}$ commute, and $I+f_{2}^{\prime}(0)^{-1} f_{2}$ commutes with $f_{1}$ and linearizes $f_{2}$. Thus $f_{j}$ can be simultaneously linearized by a holomorphic (resp. formal) change of coordinates. Without loss of generality, we may assume that each $f_{j}$ is linear. We want to diagonalize all $f_{j}$ simultaneously. Let $E_{i}^{1}$ and $E_{i}^{-1}$ be the eigenspaces of $f_{i}$ with eigenvalues 1 and -1 , respectively. Since $f_{i}=f_{j}^{-1} f_{i} f_{j}$, each eigenspace of $f_{i}$ is invariant under $f_{j}$. Then we can decompose

$$
\mathbf{C}^{n}=\bigoplus_{\left(i_{1}, \ldots, i_{s}\right)} E_{1}^{i_{1}} \cap \cdots \cap E_{s}^{i_{s}}, \quad s=2^{\ell}
$$

Here $\left(i_{1}, \ldots, i_{s}\right)$ runs over $\{-1,1\}^{s}$ with subspaces $E^{\left(i_{1}, \ldots, i_{s}\right)}:=E_{1}^{i_{1}} \cap \cdots \cap E_{s}^{i_{s}}$. On each of these subspaces, $f_{j}=I$ or $-I$. We are ready to choose a new basis for $\mathbf{C}^{n}$ whose elements are in the subspaces. Under the new basis, all $f_{j}$ are diagonal.

Let us rewrite (2.12) as $\mathbf{C}^{n}=V_{1} \oplus V_{2} \oplus \cdots \oplus V_{d}$. Here $V_{j}=E^{I_{j}}$ and $I_{1}=(1, \ldots, 1)$. Also, $I_{j} \neq(1, \ldots, 1)$ and $\operatorname{dim} V_{j}>0$ for $j>1$. We have $\operatorname{dim}_{\mathbf{C}} \operatorname{Fix}(G)=\operatorname{dim}_{\mathbf{C}} V_{1}=$ $\operatorname{dim}_{\mathbf{C}}\left[\mathfrak{M}_{n}\right]_{1}^{L G}=k$. Therefore, $d-1 \leq n-\operatorname{dim}_{\mathbf{C}} V_{1} \leq n-k$. We have proved that in suitable coordinates $G$ is contained in the group generated by $Z_{k+1}, \ldots, Z_{n}$. The remaining assertions follow easily.

We will need an elementary result about invariant functions.

Lemma 2.5. Let $Z_{k+1}, \ldots, Z_{n}$ be defined by (2.10). Let $F=\left\{f_{k+1}, \ldots, f_{n}\right\}$ be a family of germs of holomorphic mappings at the origin $0 \in \mathbf{C}^{n}$. Suppose that the family $F$ is holomorphically equivalent to $\left\{Z_{k+1}, \ldots, Z_{n}\right\}$. Let $b_{1}(z), \ldots, b_{n}(z)$ be germs of holomorphic functions that are invariant under $F$. Suppose that for $1 \leq j \leq k, b_{j}(0)=0$ and the linear part of $b_{j}$ at the origin is $\tilde{b}_{j}$. Suppose that for $i>k, b_{i}(z)=O\left(|z|^{2}\right)$ and the quadratic part of $b_{i}$ at the origin is $b_{i}^{*}$. Suppose that $\tilde{b}_{1}, \ldots, \tilde{b}_{k}$ are linear independent, and that $b_{k+1}^{*}, \ldots, b_{n}^{*}$ are linearly independent modulo $\tilde{b}_{1}, \ldots, \tilde{b}_{k}$, i.e.

$$
\sum c_{i} b_{i}^{*}(z)=\sum d_{j}(z) \tilde{b}_{j}(z)+O\left(|z|^{3}\right)
$$

holds for some constants $c_{i}$ and formal power series $d_{j}$, if and only if all $c_{i}$ are zero. Then invariant functions of $F$ are power series in $b_{1}, \ldots, b_{n}$. Furthermore, $F$ is uniquely determined by $b_{1}, \ldots, b_{n}$. The same conclusion holds if $F$ and $b_{j}$ are given by formal power series.

Proof. Without loss of generality, we assume that $F$ is $\left\{Z_{k+1}, \ldots, Z_{n}\right\}$. Hence, for all $j$, there is a formal power series $a_{j}$ such that $b_{j}(z)=a_{j}\left(z_{1}, \ldots, z_{k}, z_{k+1}^{2}, \ldots, z_{n}^{2}\right)$. Let us show that the map $w \rightarrow a(w)=\left(a_{1}(w), \ldots, a_{n}(w)\right)$ is invertible.

By Lemma 2.4, $\tilde{b}_{1}(z), \ldots, \tilde{b}_{k}(z)$ are linear combinations of $z_{1}, \ldots, z_{k}$, and vice versa. By Lemma 2.4 again, $b_{k+1}^{*}, \ldots, b_{n}^{*}$ are linear combinations of $z_{k+1}^{2}, \ldots, z_{n}^{2}$ modulo $z_{1}, \ldots, z_{k}$. This shows that

$$
b_{i}^{*}(z)=\sum_{j>k} c_{i j} z_{j}^{2}+\sum_{\ell \leq k} d_{i \ell}(z) \tilde{b}_{\ell}(z), \quad i>k .
$$

Since $b_{k+1}^{*}, \ldots, b_{n}^{*}$ are linearly independent modulo $\tilde{b}_{1}, \ldots, \tilde{b}_{k}$. Then $\left(c_{i j}\right)$ is invertible; so is the linear part of $a$. 
To show that $F$ is uniquely determined by its invariant functions, let $\tilde{F}$ be another such family that is equivalent to $\left\{Z_{k+1}, \ldots, Z_{n}\right\}$. Assume that $F$ and $\tilde{F}$ have the same invariant functions. Without loss of generality, assume that $\tilde{F}$ is $\left\{Z_{k+1}, \ldots, Z_{n}\right\}$. Then $z_{1}, \ldots, z_{k}$ are invariant by each $F_{j}$, i.e. the $i$ th component of $F_{j}(z)$ is $z_{i}$ for $i \leq k$. Also $F_{j, \ell}^{2}(z)=z_{\ell}^{2}$ for $\ell>k$. We get $F_{j, \ell}= \pm z_{\ell}$. Since $z_{\ell}$ is not invariant by $\tilde{F}$, then it is not invariant by $F$ either. Then $F_{j_{\ell}, \ell}(z)=-z_{\ell}$ for some $\ell_{j}>k$. Since $F_{j_{\ell}}$ is equivalent to some $Z_{i}$, the set of fixed points of $F_{j_{\ell}}$ is a hypersurface. This shows that $F_{j_{\ell}}=Z_{\ell}$. So the family $F$ is $\left\{Z_{k+1}, \ldots, Z_{n}\right\}$.

We now want to find a special set of generators for the deck transformations and its basic properties, which will be important to our study of the normal form problems.

Lemma 2.6. Let $M$ be defined by (2.1) and (2.2) with $q_{*}=0$. Suppose that the group $\mathcal{T}_{i}$ of deck transformations of $\pi_{i}: \mathcal{M} \rightarrow \mathbf{C}^{p}$ has exactly $2^{p}$ elements. Then the followings hold.

(i) $\mathcal{T}_{1}$ is generated by $p$ distinct involutions $\tau_{1 j}$ such that $\operatorname{Fix}\left(\tau_{11}\right), \ldots, \operatorname{Fix}\left(\tau_{1 p}\right)$ are hypersurfaces intersecting transversally at 0 . And $\tau_{1}=\tau_{11} \cdots \tau_{1 p}$ is the unique deck transformation of which the set of fixed points has dimension $p$. Moreover, $\operatorname{Fix}\left(\tau_{1}\right)=\bigcap \operatorname{Fix}\left(\tau_{1 j}\right)$.

(ii) $\mathcal{O}_{n}^{\mathcal{T}_{1}}$ (resp. $\widehat{\mathcal{O}}_{n}^{\mathcal{T}_{1}}$ ) is precisely the set of convergent (resp. formal) power series in $z^{\prime}$ and $E\left(z^{\prime}, w^{\prime}\right) . \mathcal{O}_{n}^{\mathcal{T}_{2}}$ (resp. $\left.\widehat{\mathcal{O}}_{n}^{\mathcal{T}_{2}}\right)$ is the set of convergent (resp. formal) power series in $w^{\prime}$ and $\bar{E}\left(w^{\prime}, z^{\prime}\right)$. In particular, in $\left(z^{\prime}, w^{\prime}\right)$ coordinates of $\mathcal{M}, \mathcal{T}_{1}$ and $\mathcal{T}_{2}$ satisfy

$$
\begin{gathered}
{\left[\mathfrak{M}_{n}\right]_{1}^{L \mathcal{T}_{1}} \cap\left[\mathfrak{M}_{n}\right]_{1}^{L \mathcal{T}_{2}}=\{0\}} \\
\operatorname{dim} \operatorname{Fix}\left(\tau_{i}\right)=p, \quad \operatorname{Fix}\left(\tau_{1}\right) \cap \operatorname{Fix}\left(\tau_{2}\right)=\{0\}
\end{gathered}
$$

Here $\left[\mathfrak{M}_{n}\right]_{1}$ is the set of linear functions in $z^{\prime}, w^{\prime}$ without constant terms.

Proof. (i). Since $z_{1}, \ldots, z_{p}$ are invariant under deck transformations of $\pi_{1}$, we have $p^{\prime}=$ $\operatorname{dim}_{\mathbf{C}}\left[\mathcal{O}_{n}\right]_{1}^{L \mathcal{T}_{1}} \geq p$. By Lemma 2.4, $\pi_{1}$ has at most $2^{2 p-p^{\prime}}$ deck transformations. Therefore, $p^{\prime}=p$. By Lemma 2.4 again, we may assume that in suitable $(\xi, \eta)$ coordinates, the deck transformations are generated by $Z_{p+1}, \ldots, Z_{2 p}$ defined by (2.10) in which $z=(\xi, \eta)$. It follows that $Z=Z_{p+1} \cdots Z_{2 p}$ is the unique deck transformation of $\pi_{1}$, of which the set of fixed points has dimension $p$.

(ii). We have proved that in $(\xi, \eta)$ coordinates the deck transformations are generated by the above $Z_{p+1}, \ldots, Z_{2 p}$. Thus, the invariant holomorphic functions of $Z_{p+1}, \ldots, Z_{2 p}$ are precisely the holomorphic functions in $\xi_{1}, \ldots, \xi_{p}, \eta_{1}^{2}, \ldots, \eta_{p}^{2}$. Since $z_{1}, \ldots, z_{p}$ and $E_{i}\left(z^{\prime}, w^{\prime}\right)$ are invariant under deck transformations, then on $\mathcal{M}$

$$
z^{\prime}=f\left(\xi, \eta_{1}^{2}, \ldots, \eta_{p}^{2}\right), \quad E\left(z^{\prime}, w^{\prime}\right)=g\left(\xi, \eta_{1}^{2}, \ldots, \eta_{p}^{2}\right) .
$$

Since $\left(z^{\prime}, w^{\prime}\right)$ are local coordinates of $\mathcal{M}$, the differentials of $z_{1}, \ldots, z_{p}$ under any coordinate system of $\mathcal{M}$ are linearly independent. Computing the differentials of $z^{\prime}$ in variables $\xi, \eta$ by using (2.14), we see that the mapping $\xi \rightarrow f(\xi, 0)$ is a local biholomorphism. Expressing both sides of the second identity in (2.14) as power series in $\xi, \eta$, we obtain

$$
E\left(f(\xi, 0), w^{\prime}\right)=g\left(\xi, \eta_{1}^{2}, \ldots, \eta_{p}^{2}\right)+O\left(|(\xi, \eta)|^{3}\right)
$$


We set $\xi=0$, compute the left-hand side, and rewrite the identity as

$$
g\left(0, \eta_{1}^{2}, \ldots, \eta_{p}^{2}\right)=q\left(w^{\prime}\right)+O\left(|(\xi, \eta)|^{3}\right) .
$$

As coordinate systems, $\left(z^{\prime}, w^{\prime}\right)$ and $(\xi, \eta)$ vanish at $0 \in \mathcal{M}$. We now use $\left(z^{\prime}, w^{\prime}\right)=$ $O(|(\xi, \eta)|)$. By (2.14), $f(0)=g(0)=0$ and $g(\xi, 0)=O\left(|\xi|^{2}\right)$. Let us verify that the linear parts of $g_{1}(0, \eta), \ldots, g_{p}(0, \eta)$ are linearly independent. Suppose that $\sum_{j=1}^{p} c_{j} g_{j}(0, \eta)=$ $O\left(|\eta|^{2}\right)$. Replacing $\xi, \eta$ by $O\left(\left|\left(z^{\prime}, w^{\prime}\right)\right|\right)$ in (2.15) and setting $z^{\prime}=0$, we obtain

$$
\sum_{j=1}^{p} c_{j} q_{j}\left(w^{\prime}\right)=O\left(\left|w^{\prime}\right|^{3}\right), \quad \text { i.e. } \quad \sum_{j=1}^{p} c_{j} q_{j}\left(w^{\prime}\right)=0 .
$$

As remarked after condition B was introduced, $q_{*}=0$ implies that $q_{1}\left(w^{\prime}\right), \ldots, q_{p}\left(w^{\prime}\right)$ are linearly independent. Thus all $c_{j}$ are 0 . We have verified that $\xi \rightarrow f(\xi, 0)$ is biholomorphic near $\xi=0$. Also $\eta \rightarrow g(0, \eta)$ is biholomorphic near $\eta=0$ and $g(\xi, 0)=O\left(|\xi|^{2}\right)$. Therefore, $(\xi, \eta) \rightarrow(f, g)(\xi, \eta)$ is invertible near 0 . By solving (2.14), the functions $\xi, \eta_{1}^{2}, \ldots, \eta_{p}^{2}$ are expressed as power series in $z^{\prime}$ and $E\left(z^{\prime}, w^{\prime}\right)$.

It is clear that $z_{1}, \ldots, z_{p}$ are invariant under $\tau_{1 j}$. From linearization of $\mathcal{T}_{1}$, we know that the space of invariant linear functions of $L \mathcal{T}_{1}$ is the same as the space of linear invariant functions of $L \tau_{1}$, which has dimension $p$. This shows that $z_{1}, \ldots, z_{p}$ span the space of linear invariant functions of $L \tau_{1}$. Also $w_{1}, \ldots, w_{p}$ span the space of linear invariant functions of $L \tau_{2}$. We obtain $\left[\mathfrak{M}_{n}\right]_{1}^{L \mathcal{T}_{1}} \cap\left[\mathfrak{M}_{n}\right]_{1}^{L \mathcal{T}_{2}}=\{0\}$. We have verified $(2.13)$.

In view of the linearization of $\mathcal{T}_{1}$ in (i), we obtain $\operatorname{dim} \operatorname{Fix}\left(\tau_{1}\right)=\operatorname{dim} \operatorname{Fix}\left(\mathcal{T}_{1}\right)=p$. Moreover, $\operatorname{Fix}\left(\tau_{i}\right)$ is a smooth submanifold of which the tangent space at the origin is $\operatorname{Fix}\left(L \tau_{i}\right)$. We choose a basis $u_{1}, \ldots, u_{p}$ for $\operatorname{Fix}\left(L \tau_{1}\right)$. Let $v_{1}, \ldots, v_{p}$ be any $p$ vectors such that $u_{1}, \ldots, u_{p}, v_{1}, \ldots, v_{p}$ form a basis of $\mathbf{C}^{n}$. In new coordinates defined by $\sum \xi_{i} u_{i}+\eta_{i} v_{i}$, we know that linear invariant functions of $L \tau_{1}$ are spanned by $\xi_{1}, \ldots, \xi_{p}$. The linear invariant functions in $(\xi, \eta)$ that are invariant by $L \tau_{2}$ are spanned by $f_{j}(\xi, \eta)=\sum_{k}\left(a_{j k} \xi_{k}+b_{j k} \eta_{k}\right)$ for $1 \leq j \leq p$. Since $\left[\mathfrak{M}_{n}\right]^{L \tau_{1}} \cap\left[\mathfrak{M}_{n}\right]^{L \tau_{2}}=\{0\}$, then $\xi_{1}, \ldots, \xi_{p}, f_{1}, \ldots, f_{p}$ are linearly independent. Equivalently, $\left(b_{j k}\right)$ is non-singular. Now $\operatorname{Fix}\left(L \tau_{2}\right)$ is spanned by vectors $\sum_{k}\left(a_{j k} u_{k}+b_{j k} v_{k}\right)$. This shows that $\operatorname{Fix}\left(L \tau_{1}\right) \cap \operatorname{Fix}\left(L \tau_{2}\right)=\{0\}$. Therefore, $\operatorname{Fix}\left(\tau_{1}\right)$ intersects $\operatorname{Fix}\left(\tau_{2}\right)$ transversally at the origin and the intersection must be the origin.

Note that the proof of the above lemma actually gives us a more general result.

Corollary 2.7. Let $\mathfrak{I}$ be a group of commuting holomorphic (formal) involutions on $\mathbf{C}^{n}$.

(i) $\operatorname{Fix}(L \mathfrak{I})=\{0\}$ if and only if $\left[\mathfrak{M}_{n}\right]_{1}^{L \mathfrak{I}}$ has dimension 0 .

(ii) Let $\widetilde{\mathfrak{I}}$ be another family of commuting holomorphic (resp. formal) involutions such that $\left[\mathfrak{M}_{n}\right]^{L \mathfrak{I}} \cap\left[\mathfrak{M}_{n}\right]^{L \widetilde{I}}=\{0\}$. Then $\operatorname{Fix}(L \mathfrak{I}) \cap \operatorname{Fix}(L \widetilde{\mathfrak{I}})=\{0\}$. Moreover, $\operatorname{Fix}(\mathfrak{I}) \cap$ $\operatorname{Fix}(\widetilde{\mathfrak{I}})=\{0\}$ if $\mathfrak{I}$ and $\widetilde{\mathfrak{I}}$ consist of convergent involutions.

In view of Lemma 2.6, we will refer to the family

$$
\mathcal{T}_{1}:=\left\{\tau_{11}, \ldots, \tau_{1 p}\right\}, \quad \mathcal{T}_{2}:=\left\{\tau_{21}, \ldots, \tau_{2 p}\right\}, \quad \rho
$$

as the Moser-Webster involutions, where $\tau_{2 j}=\rho \tau_{1 j} \rho$. The significance of the two sets of involutions is the following proposition that transforms the normalization of the real manifolds into that of two families of commuting involutions.

For clarity, recall the anti-holomorphic involution $\rho_{0}:\left(z^{\prime}, w^{\prime}\right) \rightarrow\left(\overline{w^{\prime}}, \overline{z^{\prime}}\right)$. 
Proposition 2.8. Let $M$ and $\widetilde{M}$ be two real analytic submanifolds of the form (2.1) and (2.2) that admit Moser-Webster involutions $\left\{\mathcal{T}_{1}, \rho_{0}\right\}$ and $\left\{\widetilde{\mathcal{T}}_{1}, \rho_{0}\right\}$, respectively. Then $M$ and $\widetilde{M}$ are holomorphically equivalent if and only if $\left\{\mathcal{T}_{1}, \rho_{0}\right\}$ and $\left\{\widetilde{\mathcal{T}}_{1}, \rho_{0}\right\}$ are holomorphically equivalent, i.e. if there is a biholomorphic map $f$ commuting with $\rho_{0}$ such that $f \tau_{1 j} f^{-1}=$ $\tilde{\tau}_{1 i_{j}}$ for $1 \leq j \leq p$. Here $\left\{i_{1}, \ldots, i_{p}\right\}=\{1, \ldots, p\}$.

Let $\mathcal{T}_{1}=\left\{\tau_{11}, \ldots, \tau_{1 p}\right\}$ be a family of $p$ distinct commuting holomorphic involutions. Suppose that $\operatorname{Fix}\left(\tau_{11}\right), \ldots, \operatorname{Fix}\left(\tau_{1 p}\right)$ are hypersurfaces intersecting transversely at the origin. Let $\rho$ be an anti-holomorphic involutions and let $\mathcal{T}_{2}$ be the family of involutions $\tau_{2 j}=\rho \tau_{1 j} \rho$ with $1 \leq j \leq p$. Suppose that

$$
\left[\mathfrak{M}_{n}\right]_{1}^{L \mathcal{T}_{1}} \cap\left[\mathfrak{M}_{n}\right]_{1}^{L \mathcal{T}_{2}}=\{0\}
$$

There exists a real analytic real $n$-submanifold

$$
M \subset \mathbf{C}^{2 p}: z_{p+j}=A_{j}^{2}\left(z^{\prime}, \bar{z}^{\prime}\right), \quad 1 \leq j \leq p
$$

such that the set of Moser-Webster involutions $\left\{\widetilde{\mathcal{T}}_{1}, \rho_{0}\right\}$ of $M$ is holomorphically equivalent to $\left\{\mathcal{T}_{1}, \rho\right\}$.

Proof. We recall from (2.5) the branched covering

$$
\pi_{1}: \mathcal{M}_{1}:=\mathcal{M} \cap\left(\left(\Delta_{\delta}^{p} \times \Delta_{\delta^{2}}^{p}\right) \times\left(\Delta_{C \delta}^{p} \times \Delta_{C \delta^{2}}^{p}\right)\right) \longrightarrow \Delta_{\delta}^{p} \times \Delta_{\delta^{2}}^{p}
$$

Here $C \geq 1$. Let $\pi_{1}$ be restricted to $\mathcal{M}_{1}$. Then $\pi_{2}=\overline{\pi_{1} \circ \rho}$ is defined on $\rho\left(\mathcal{M}_{1}\right)$. Note that

$$
\pi_{2}: \rho\left(\mathcal{M}_{1}\right) \longrightarrow \Delta_{\delta}^{p} \times \Delta_{\delta^{2}}^{p}
$$

We have $\pi_{1}^{-1}(z) \cap \operatorname{Fix}(\rho)=\{(z, \bar{z})\}$ for $z \in M$ and $\pi_{1}(\operatorname{Fix}(\rho))=M$. Let $\mathcal{B}_{0} \subset \Delta_{\delta}^{p} \times \Delta_{\delta^{2}}^{p}$ be the branched locus. Take $\mathcal{B}=\pi_{1}^{-1}\left(\mathcal{B}_{0}\right)$. We will denote by $\widetilde{\mathcal{M}}_{1}, \tilde{\mathcal{B}}$ and $\tilde{\mathcal{B}}_{0}$ the corresponding data for $\widetilde{M}$. Here $\widetilde{\mathcal{M}}_{1}$ is an analogous branched covering over $\pi_{1}\left(\widetilde{\mathcal{M}}_{1}\right)$. We assume that the latter contains $f\left(\pi_{1}\left(\mathcal{M}_{1}\right)\right)$ if $\widetilde{M}$ is equivalent to $M$ via $f$.

Assume that $f$ is a biholomorphic map sending $M$ into $\widetilde{M}$. Let $f^{c}$ be the restriction of biholomorphic map $f^{c}(z, w)=(f(z), \bar{f}(w))$ to $\mathcal{M}$. Let $M$ be defined by $z^{\prime \prime}=E\left(z^{\prime}, \bar{z}^{\prime}\right)$ and $\widetilde{M}$ be defined by $z^{\prime \prime}=\tilde{E}\left(z^{\prime}, \bar{z}^{\prime}\right)$. By $f(M) \subset \widetilde{M}, f=\left(f^{\prime}, f^{\prime \prime}\right)$ satisfies

$$
f^{\prime \prime}\left(z^{\prime}, E\left(z^{\prime}, \bar{z}^{\prime}\right)\right)=\tilde{E}\left(f^{\prime}\left(z^{\prime}, E\left(z^{\prime}, \bar{z}^{\prime}\right)\right), \overline{f^{\prime}}\left(\bar{z}^{\prime}, \bar{E}\left(\bar{z}^{\prime}, z^{\prime}\right)\right)\right)
$$

Using the defining equations for $\mathcal{M}$, we get $f^{c}(\mathcal{M}) \subset \widetilde{\mathcal{M}}$ and $\rho f^{c}=f^{c} \rho$ on $\mathcal{M} \cap \rho(\mathcal{M})$. We will also assume that $f^{c}\left(\mathcal{M}_{1}\right)$ is contained in $\widetilde{\mathcal{M}}_{1}$. It is clear that $f^{c}$ sends a fiber $\pi_{1}^{-1}(z)$ onto the fiber $\pi_{1}^{-1}(f(z))$ for $z \in \Omega=\pi_{1}\left(\mathcal{M}_{1}\right) \backslash\left(\mathcal{B}_{0} \cup f^{-1}\left(\tilde{B}_{0}\right)\right)$, since the two fibers have the same number of points and $f$ is injective. Thus $f^{c} \tau_{1 j}=\tilde{\tau}_{1 i_{j}} f^{c}$ on $\pi_{1}^{-1}(\Omega)$. Here $i_{j}$ is of course locally determined on $\pi_{1}^{-1}(\Omega)$. Since $\mathcal{B}$ has positive codimension in $\mathcal{M}_{1}$ then $\mathcal{M}_{1} \backslash \mathcal{B}$ is connected. Hence $i_{j}$ is well-defined on $\pi_{1}^{-1}(\Omega)$. Then $f^{c} \tau_{1 j}=\tilde{\tau}_{1 i_{j}} f^{c}$ on $\mathcal{M}_{1} \backslash B$. This shows that $f^{c}$ conjugates simultaneously the deck transformations of $\mathcal{M}$ to the deck transformations of $\widetilde{\mathcal{M}}$ for $\pi_{1}$. The same conclusion holds for $\pi_{2}$.

Conversely, assume that there is a biholomorphic map $g: \mathcal{M} \rightarrow \widetilde{\mathcal{M}}$ such that $\rho g=g \rho$ and $g \tau_{1 i}=\tilde{\tau}_{1 j_{i}} g$. Since $\tau_{11}, \ldots, \tau_{12^{p}}$ are distinct and $M_{1} \backslash \mathcal{B}$ is connected, then $\bigcup_{j \neq i}\left\{x \in \mathcal{M}_{1} \backslash\right.$ $\left.\mathcal{B}: \tau_{1 i}(x)=\tau_{1 j}(x)\right\}$ is a complex subvariety of positive codimension in $\mathcal{M}_{1} \backslash \mathcal{B}$. Its image under the proper projection $\pi_{1}$ is a subvariety of positive codimension in $\Delta_{\delta}^{p} \times \Delta_{\delta^{2}}^{p} \backslash \mathcal{B}_{0}$. This 
shows that the latter contains a non-empty open subset $\omega$ such that $\left\{\tau_{11}(x), \ldots, \tau_{12^{p}}(x)\right\}=$ $\pi_{1}^{-1} \pi_{1}(x)$ has $2^{p}$ distinct points for each $\pi_{1}(x) \in \omega$. Therefore, $\tau_{11}, \ldots, \tau_{12^{p}}$ are all deck transformations of $\pi_{1}$ over $\omega$. Hence they are all deck transformations of $\pi_{1}: \mathcal{M}_{1} \backslash \mathcal{B} \rightarrow$ $\Delta_{\delta}^{p} \times \Delta_{\delta^{2}}^{p} \backslash \mathcal{B}_{0}$, too. This shows that $\pi_{1}^{-1}\left(\pi_{1}(x)\right)=\left\{\tau_{1 j}(x): 1 \leq j \leq 2^{p}\right\}$ for $x \in \mathcal{M}_{1} \backslash \mathcal{B}$. Now, $g$ sends $\tau_{1 j}(x)$ to $\tilde{\tau}_{1 i_{j}}(g(x))$ for each $j$. Hence $f(z)=\pi_{1} g \pi_{1}^{-1}(z)$ is well-defined and holomorphic for $z \in \Delta_{\delta}^{p} \times \Delta_{\delta^{2}}^{p} \backslash \mathcal{B}_{0}$. By the Riemann extension for bounded holomorphic functions, $f$ extends to a holomorphic mapping, still denoted by $f$, which is defined near the origin. We know that $f$ is invertible and in fact the inverse can be obtained by extending the mapping $z \rightarrow \pi_{1} g^{-1} \pi_{1}(z)$. If $z=\left(z^{\prime}, E\left(z^{\prime}, w^{\prime}\right)\right) \in M$, then $w^{\prime}=\bar{z}^{\prime}$ and $f(z)=$ $\pi_{1} g \pi_{1}^{-1}(z)=\pi_{1} g(z, \bar{z})$ with $(z, \bar{z}) \in \operatorname{Fix}(\rho)$. Since $\rho g=g \rho$, then $g(z, \bar{z}) \in \operatorname{Fix}(\rho)$. Thus $f(z)=\pi_{1} g(z, \bar{z}) \in \widetilde{M}$.

Assume that $\left\{\tau_{1 j}\right\}$ and $\rho$ are germs of involutions defined at the origin of $\mathbf{C}^{n}$. Assume that they satisfy the conditions in the proposition. From Lemma 2.4 it follows that $\tau_{11}, \ldots, \tau_{1 p}$ generate a group of $2^{p}$ involutions, while the $p$ generators are the only elements of which each fixes a hypersurface pointwise. To realize them as deck transformations of the complexification of a real analytic submanifold, we apply Lemma 2.4 to find a coordinate $\operatorname{map}(\xi, \eta) \rightarrow \phi(\xi, \eta)=(A, B)(\xi, \eta)$ such that invariant holomorphic functions of $\left\{\tau_{1 j}\right\}$ are precisely holomorphic functions in

$$
z^{\prime}=\left(A_{1}(\xi, \eta), \ldots, A_{p}(\xi, \eta)\right), \quad z^{\prime \prime}=\left(B_{1}^{2}(\xi, \eta), \ldots, B_{p}^{2}(\xi, \eta)\right) .
$$

Note that $B_{j}$ is skew-invariant under $\tau_{1 j}$ and is invariant under $\tau_{1 i}$ for $i \neq j$ and $A$ is invariant under all $\tau_{1 j}$. Set

$$
w_{j}^{\prime}=\overline{A_{j} \circ \rho(\xi, \eta)}, \quad w_{j}^{\prime \prime}=\overline{B_{j}^{2} \circ \rho(\xi, \eta)} .
$$

Since $\tau_{2 j}=\rho \tau_{1 j} \rho$, the holomorphic functions invariant under all $\tau_{2 j}$ are precisely the holomorphic functions in the above $w^{\prime}, w^{\prime \prime}$. We now draw conclusions for the linear parts of invariant functions and involutions. Since $\phi$ is biholomorphic, then $L A_{1}, \ldots, L A_{p}$ are linearly independent. They are also invariant under $L \tau_{1 j}$. Since $\tau_{2 j}=\rho \tau_{1 j} \rho$, the $p$ functions $L \overline{A_{i} \circ \rho}$ are linearly independent and invariant under $L \tau_{2 j}$. Thus

$$
L A_{1}, \ldots, L A_{p}, L \overline{A_{1} \circ \rho}, \ldots, L \overline{A_{p} \circ \rho}
$$

are linearly independent, since $\left[\mathfrak{M}_{n}\right]_{1}^{L \mathcal{T}_{1}} \cap\left[\mathfrak{M}_{n}\right]_{1}^{L \mathcal{T}_{2}}=\{0\}$. This shows that the map $(\xi, \eta) \rightarrow$ $\left(z^{\prime}, w^{\prime}\right)=(A(\xi, \eta), \overline{A \circ \rho(\xi, \eta)})$ has an inverse $(\xi, \eta)=\psi\left(z^{\prime}, w^{\prime}\right)$. Define

$$
M: z^{\prime \prime}=\left(B_{1}^{2}, \ldots, B_{p}^{2}\right) \circ \psi\left(z^{\prime}, \bar{z}^{\prime}\right) .
$$

The complexification of $M$ is given by

$$
\mathcal{M}: z^{\prime \prime}=\left(B_{1}^{2}, \ldots, B_{p}^{2}\right) \circ \psi\left(z^{\prime}, w^{\prime}\right), \quad w^{\prime \prime}=\left(\bar{B}_{1}^{2}, \ldots, \bar{B}_{p}^{2}\right) \circ \bar{\psi}\left(w^{\prime}, z^{\prime}\right) .
$$

Note that $\phi \circ \psi\left(z^{\prime}, w^{\prime}\right)=\left(z^{\prime}, B \circ \psi\left(z^{\prime}, w^{\prime}\right)\right)$ is biholomorphic. In particular, we can write

$$
B_{j}^{2} \circ \psi\left(z^{\prime}, \bar{z}^{\prime}\right)=h_{j}\left(z^{\prime}, \bar{z}^{\prime}\right)+q_{j}\left(\bar{z}^{\prime}\right)+b_{j}\left(z^{\prime}\right)+O\left(\left|\left(z^{\prime}, \bar{z}^{\prime}\right)\right|^{3}\right) .
$$

Here $q_{j}\left(\bar{z}^{\prime}\right)=\tilde{q}_{j}^{2}\left(\bar{z}^{\prime}\right)$, and $\tilde{q}\left(w^{\prime}\right)$ is the linear part of $w^{\prime} \rightarrow B \circ \psi\left(0, w^{\prime}\right)$. Therefore, $\left|q\left(w^{\prime}\right)\right| \geq$ $c\left|w^{\prime}\right|^{2}$ and $q_{*}=0$. By Lemma 2.1, $\pi_{1}: \mathcal{M} \rightarrow \mathbf{C}^{p}$ is a $2^{p}$-to-1 branched covering defined near $0 \in \mathcal{M}$. Since $B^{2}$ is invariant by $\tau_{1 j}$, then $z^{\prime \prime}=B^{2} \circ \psi\left(z^{\prime}, w^{\prime}\right)$ is invariant by $\psi^{-1} \tau_{1 j} \psi\left(z^{\prime}, w^{\prime}\right)$. Also $A$ is invariant under $\tau_{1 j}$. Then $z^{\prime}=A \circ \psi\left(z^{\prime}, w^{\prime}\right)$ is invariant by $\psi^{-1} \tau_{1 j} \psi\left(z^{\prime}, w^{\prime}\right)$. This 
show that $\left\{\psi^{-1} \tau_{1 j} \psi\right\}$ has the same invariant functions as of the deck transformations of $\pi_{1}$. By Lemma 2.5, $\left\{\psi^{-1} \tau_{1 j} \psi\right\}$ agrees with the set of deck transformations of $\pi_{1}$. For $\rho_{0}\left(z^{\prime}, w^{\prime}\right)=$ $\left(\overline{w^{\prime}}, \overline{z^{\prime}}\right)$ we have $\rho_{0} \psi^{-1}=\psi^{-1} \rho$. This shows that $M$ is a realization for $\left\{\tau_{11}, \ldots, \tau_{1 p}, \rho\right\}$.

Remark 2.9. We choose the realization in such a way that $z_{p+j}$ are square functions. This particular holomorphic equivalent form of $M$ will be crucial to study the asymptotic manifolds in section 7.

Next we want to compute the deck transformations for a product quadric. We will first recall the Moser-Webster involutions for elliptic and hyperbolic complex tangents. We will then compute the deck transformations for complex tangents of complex type.

The Moser-Webster theory deals with the case $p=1$ for a real analytic surface

$$
z_{2}=\left|z_{1}\right|^{2}+\gamma\left(z_{1}^{2}+\bar{z}_{1}^{2}\right)+O\left(\left|z_{1}\right|^{3}\right), \quad \text { or } \quad z_{2}=z_{1}^{2}+\bar{z}_{1}^{2}+O\left(\left|z_{1}\right|^{3}\right) .
$$

Here $\gamma \geq 0$ is the Bishop invariant of $M$. One of most important properties of the MoserWebster theory is the existence of the above mentioned deck transformations. When $\gamma \neq 0$, there is a pair of Moser-Webster involutions $\tau_{1}, \tau_{2}$ with $\tau_{2}=\rho \tau_{1} \rho$ such that $\tau_{1}$ generates the deck transformations of $\pi_{1}$. In fact, $\tau_{1}$ is the only possible non-trivial deck transformation of $\pi_{1}$. When $\gamma \neq 1 / 2$, in suitable coordinates their composition $\sigma=\tau_{1} \tau_{2}$ is of the form

$$
\tau: \xi^{\prime}=\mu \xi+O\left(|(\xi, \eta)|^{2}\right), \quad \eta^{\prime}=\mu^{-1} \eta+O\left(|(\xi, \eta)|^{2}\right) .
$$

Here $\rho(\xi, \eta)=(\bar{\eta}, \bar{\xi})$ when $0<\gamma<1 / 2$, and $\rho(\xi, \eta)=(\bar{\xi}, \bar{\eta})$ when $\gamma>1 / 2$. When the complex tangent is elliptic and $0<\gamma<1 / 2, \sigma$ is hyperbolic with $\mu>1$; when the complex tangent is hyperbolic, i.e. $1 / 2<\gamma \leq \infty$, then $\sigma$ is elliptic with $|\mu|=1$. When the complex tangent is parabolic, the linear part of $\sigma$ is not diagonalizable and 1 is the eigenvalue.

We will see later that with $p \geq 2$, there is yet another simple model that is not in the product. This is the quadric in $\mathbf{C}^{4}$ defined by

$$
Q_{\gamma_{s}}: z_{3}=z_{1} \bar{z}_{2}+\gamma_{s} \bar{z}_{2}^{2}+\left(1-\gamma_{s}\right) z_{1}^{2}, \quad z_{4}=\bar{z}_{3}
$$

Here $\gamma_{s}$ is a complex number. We will, however, exclude $\gamma_{s}=0$ or equivalently $\gamma_{s}=1$ by condition B. We also exclude $\gamma_{s}=1 / 2$ by condition J. Note that $\gamma_{s}=1 / 2$ does not correspond to a product Bishop quadrics either, by examining the CR singular sets. Under these mild non degeneracy conditions, we will show that $\gamma_{s}$ is an invariant when it is normalized to the range

$$
\operatorname{Re} \gamma_{s} \leq 1 / 2, \quad \operatorname{Im} \gamma_{s} \geq 0, \quad \gamma_{s} \neq 0
$$

In this case, the complex tangent is said of complex type.

We have introduced the types of the complex tangent at the origin. Of course a product of quadrics, or a product quadric, can exhibit a combination of the above basic 4 types. We will see soon that quadrics have other invariants when $p>1$. Nevertheless, in our results, the above invariants that describe the types of the complex tangent will play a major role in the convergence or divergence of normalizations.

Let us first recall involutions in [MW83] where the complex tangents are elliptic (with non-vanishing Bishop invariant) or hyperbolic. When $\gamma>0$, the non-trivial deck transformations of

$$
Q_{\gamma}: z_{2}=\left|z_{1}\right|^{2}+\gamma\left(z_{1}^{2}+\bar{z}_{1}^{2}\right)
$$


for $\pi_{1}, \pi_{2}$ are $\tau_{1}, \tau_{2}$, respectively. They are

$$
\tau_{1}: z_{1}^{\prime}=z_{1}, \quad w_{1}^{\prime}=-w_{1}-\gamma^{-1} z_{1} ; \quad \tau_{2}=\rho \tau_{1} \rho
$$

with $\rho$ being defined by (2.4). Here the formula is valid for $\gamma=\infty$ (i.e. $\gamma^{-1}=0$ ). Note that $\tau_{1}$ and $\tau_{2}$ do not commute and $\sigma=\tau_{1} \tau_{2}$ satisfies

$$
\sigma^{-1}=\tau_{i} \sigma \tau_{i}=\rho \sigma \rho, \quad \tau_{i}^{2}=I, \quad \rho^{2}=I .
$$

When the complex tangent is not parabolic, the eigenvalues of $\sigma$ are $\mu, \mu^{-1}$ with $\mu=\lambda^{2}$ and $\gamma \lambda^{2}-\lambda+\gamma=0$. For the elliptic complex tangent, we can choose a solution $\lambda>1$, and in suitable coordinates we obtain

$$
\begin{gathered}
\tau_{1}: \xi^{\prime}=\lambda \eta+O\left(|(\xi, \eta)|^{2}\right), \quad \eta^{\prime}=\lambda^{-1} \xi+O\left(|(\xi, \eta)|^{2}\right), \\
\tau_{2}=\rho \tau_{1} \rho, \quad \rho(\xi, \eta)=(\bar{\eta}, \bar{\xi}), \\
\sigma: \xi^{\prime}=\mu \xi+O\left(|(\xi, \eta)|^{2}\right), \quad \eta^{\prime}=\mu^{-1} \eta+O\left(|(\xi, \eta)|^{2}\right), \quad \mu=\lambda^{2} .
\end{gathered}
$$

When the complex tangent is hyperbolic, i.e. $1 / 2<\gamma \leq \infty, \tau_{i}$ and $\sigma$ still have the above form, while $|\mu|=1=|\lambda|$ and

$$
\rho(\xi, \eta)=(\bar{\xi}, \bar{\eta})
$$

When the complex tangent is parabolic, i.e. $\gamma=1 / 2$, the pair of involutions still exists. However, $L \sigma$ is not diagonalizable and 1 is its only eigenvalue. We recall from [MW83] that

$$
\gamma=\frac{1}{\lambda+\lambda^{-1}}
$$

For the complex type, new situations arise. Such a quadric has the form

$$
Q_{\gamma_{s}}: z_{3}=z_{1} \bar{z}_{2}+\gamma_{s} \bar{z}_{2}^{2}+\left(1-\gamma_{s}\right) z_{1}^{2}, \quad z_{4}=\bar{z}_{3}
$$

Here $\gamma_{s}$ is a complex number. Let us first check that such a quadric is not the product of two Bishop quadrics : Its CR singular set is defined by

$$
\left(z_{1}+2 \gamma_{s} \bar{z}_{2}\right)\left(z_{2}+2\left(1-\bar{\gamma}_{s}\right) \bar{z}_{1}\right)=0
$$

which is the union of a complex line and a real surface when $\gamma_{s}=0,1$, or a union of two totally real surfaces. The CR singular set of a quadric defined by $z_{3}=\left|z_{1}\right|^{2}+\gamma_{1}\left(z_{1}^{2}+\bar{z}_{1}^{2}\right)$ and $z_{4}=\left|z_{2}\right|^{2}+\gamma_{2}\left(z_{2}^{2}+\bar{z}_{2}^{2}\right)$ is given by

$$
\left(z_{1}+2 \gamma_{1} \bar{z}_{1}\right)\left(z_{2}+2 \gamma_{2} \bar{z}_{2}\right)=0 \text {. }
$$

It is the union of two Bishop surfaces when $\gamma_{1} \neq 1 / 2$ and $\gamma_{2} \neq 1 / 2$, and it contains a submanifold of dimension 3 otherwise.

By condition $\mathrm{B}$, we know that $\gamma_{s} \neq 0,1$. Let us compute the deck transformations of the complexification of (2.23). According to Lemma 2.6 (i), the deck transformations for $\pi_{1}$ are generated by two involutions

$$
\tau_{11}:\left\{\begin{array}{l}
z_{1}^{\prime}=z_{1}, \\
z_{2}^{\prime}=z_{2}, \\
w_{1}^{\prime}=-w_{1}-\left(1-\bar{\gamma}_{s}\right)^{-1} z_{2}, \\
w_{2}^{\prime}=w_{2} ;
\end{array} \quad \tau_{12}:\left\{\begin{array}{l}
z_{1}^{\prime}=z_{1} \\
z_{2}^{\prime}=z_{2} \\
w_{1}^{\prime}=w_{1} \\
w_{2}^{\prime}=-w_{2}-\gamma_{s}^{-1} z_{1} .
\end{array}\right.\right.
$$


We still have $\rho$ defined by (2.4) . Let $\tau_{2 j}=\rho \tau_{1 j} \rho$. Then $\tau_{21}, \tau_{22}$ generate the deck transformations of $\pi_{2}$. Note that

$$
\tau_{21}:\left\{\begin{array}{l}
z_{1}^{\prime}=-z_{1}-\left(1-\gamma_{s}\right)^{-1} w_{2}, \\
z_{2}^{\prime}=z_{2}, \\
w_{1}^{\prime}=w_{1}, \\
w_{2}^{\prime}=w_{2} ;
\end{array} \quad \tau_{22}:\left\{\begin{array}{l}
z_{1}^{\prime}=z_{1}, \\
z_{2}^{\prime}=-z_{2}-\bar{\gamma}_{s}^{-1} w_{1}, \\
w_{1}^{\prime}=w_{1}, \\
w_{2}^{\prime}=w_{2} .
\end{array}\right.\right.
$$

Recall that $\tau_{i}=\tau_{i 1} \tau_{i 2}$ is the unique deck transformation of $\pi_{i}$ that has the smallest dimension of the set of fixed-points among all deck transformations. They are

$$
\tau_{1}:\left\{\begin{array}{l}
z_{1}^{\prime}=z_{1}, \\
z_{2}^{\prime}=z_{2}, \\
w_{1}^{\prime}=-w_{1}-\left(1-\bar{\gamma}_{s}\right)^{-1} z_{2}, \\
w_{2}^{\prime}=-w_{2}-\gamma_{s}^{-1} z_{1} ;
\end{array} \quad \tau_{2}:\left\{\begin{array}{l}
z_{1}^{\prime}=-z_{1}-\left(1-\gamma_{s}\right)^{-1} w_{2}, \\
z_{2}^{\prime}=-z_{2}-\bar{\gamma}_{s}^{-1} w_{1}, \\
w_{1}^{\prime}=w_{1}, \\
w_{2}^{\prime}=w_{2} .
\end{array}\right.\right.
$$

And $\tau_{1} \tau_{2}$ is given by

$$
\sigma_{s}:\left\{\begin{array}{l}
z_{1}^{\prime}=-z_{1}-\left(1-\gamma_{s}\right)^{-1} w_{2} \\
z_{2}^{\prime}=-z_{2}-\bar{\gamma}_{s}^{-1} w_{1} \\
w_{1}^{\prime}=\left(1-\bar{\gamma}_{s}\right)^{-1} z_{2}+\left(\left(\bar{\gamma}_{s}-\bar{\gamma}_{s}^{2}\right)^{-1}-1\right) w_{1} \\
w_{2}^{\prime}=\gamma_{s}^{-1} z_{1}+\left(\left(\gamma_{s}-\gamma_{s}^{2}\right)^{-1}-1\right) w_{2}
\end{array}\right.
$$

In contrast to the elliptic and hyperbolic cases, $\tau_{11}$ and $\rho \tau_{11} \rho$ commute; in other words, $\tau_{11} \rho \tau_{11} \rho$ is actually an involution. And $\tau_{12}$ and $\rho \tau_{12} \rho$ commute, too. However, $\tau_{11}$ and $\tau_{22}$ do not commute, and $\tau_{12}, \tau_{21}$ do not commute either. Thus, we form compositions

$$
\sigma_{s 1}=\tau_{11} \tau_{22}, \quad \sigma_{s 2}=\tau_{12} \tau_{21}, \quad \sigma_{s 2}^{-1}=\rho \sigma_{s 1} \rho .
$$

By a simple computation, we have

$$
\begin{gathered}
\sigma_{s 1}:\left\{\begin{array}{l}
z_{1}^{\prime}=z_{1}, \\
z_{2}^{\prime}=-z_{2}-\bar{\gamma}_{s}^{-1} w_{1}, \\
w_{1}^{\prime}=\left(1-\bar{\gamma}_{s}\right)^{-1} z_{2}+\left(\left(\bar{\gamma}_{s}-\bar{\gamma}_{s}^{2}\right)^{-1}-1\right) w_{1}, \\
w_{2}^{\prime}=w_{2}
\end{array}\right. \\
\sigma_{s 2}:\left\{\begin{array}{l}
z_{1}^{\prime}=-z_{1}-\left(1-\gamma_{s}\right)^{-1} w_{2}, \\
z_{2}^{\prime}=z_{2}, \\
w_{1}^{\prime}=w_{1}, \\
w_{2}^{\prime}=\gamma_{s}^{-1} z_{1}+\left(\left(\gamma_{s}-\gamma_{s}^{2}\right)^{-1}-1\right) w_{2} .
\end{array}\right.
\end{gathered}
$$

We verify that $\sigma_{s 1} \sigma_{s 2}=\sigma_{s}=\tau_{1} \tau_{2}$. This allows us to compute the eigenvalues of $\sigma_{s 1} \sigma_{s 2}$ easily:

$$
\begin{gathered}
\mu_{s}, \quad \mu_{s}^{-1}, \bar{\mu}_{s}^{-1}, \bar{\mu}_{s}, \\
\mu_{s}=\bar{\gamma}_{s}^{-1}-1 .
\end{gathered}
$$

In fact we compute them by observing that the first two in (2.25) and 1 with multiplicity are eigenvalues of $\sigma_{s 1}$, while the last two in (2.25) and 1 with multiplicity are eigenvalues of 
$\sigma_{s 2}$. Note that $\sigma_{s}$ is diagonalizable if and only if $\gamma_{s} \neq 1 / 2$. When $\gamma_{s}<1 / 2$ and $\gamma_{s} \neq 0, \mu_{s}$ and $\mu_{s}^{-1}$ are eigenvalues of multiplicity two while condition $\mathrm{J}$ holds. Therefore, for $\gamma_{s} \neq 1 / 2$, i.e. $\mu_{s} \neq 1$, we can find a linear transformation of the form

$$
\psi:\left(z_{1}, w_{2}\right) \rightarrow\left(\xi_{2}, \eta_{2}\right)=\bar{\phi}\left(z_{1}, w_{2}\right), \quad\left(z_{2}, w_{1}\right) \rightarrow\left(\xi_{1}, \eta_{1}\right)=\phi\left(w_{1}, z_{2}\right)
$$

such that $\sigma_{s 1}, \sigma_{s 2}, \sigma_{s}=\sigma_{s 1} \sigma_{s 2}$ are simultaneously diagonalized as

$$
\begin{array}{rllll}
\sigma_{s 1}: & \xi_{1}^{\prime}=\mu_{s} \xi_{1}, & \eta_{1}^{\prime}=\mu_{s}^{-1} \eta_{1}, & \xi_{2}^{\prime}=\xi_{2}, & \eta_{2}^{\prime}=\eta_{2}, \\
\sigma_{s 2}: & \xi_{1}^{\prime}=\xi_{1}, & \eta_{1}^{\prime}=\eta_{1}, & \xi_{2}^{\prime}=\bar{\mu}_{s}^{-1} \xi_{2}, & \eta_{2}^{\prime}=\bar{\mu}_{s} \eta_{2}, \\
\sigma_{s}: & \xi_{1}^{\prime}=\mu_{s} \xi_{1}, & \eta_{1}^{\prime}=\mu_{s}^{-1} \eta_{1}, & \xi_{2}^{\prime}=\bar{\mu}_{s}^{-1} \xi_{2}, & \eta_{2}^{\prime}=\bar{\mu}_{s} \eta_{2} .
\end{array}
$$

Under the transformation $\psi$, the involution $\rho$, defined by (2.4), takes the form

$$
\rho\left(\xi_{1}, \xi_{2}, \eta_{1}, \eta_{2}\right)=\left(\bar{\xi}_{2}, \bar{\xi}_{1}, \bar{\eta}_{2}, \bar{\eta}_{1}\right)
$$

Moreover, for $j=1,2$, we have $\tau_{2 j}=\rho \tau_{1 j} \rho$ and

$$
\begin{gathered}
\tau_{1 j}: \xi_{j}^{\prime}=\lambda_{j} \eta_{j}, \quad \eta_{j}^{\prime}=\lambda_{j}^{-1} \xi_{j} ; \quad \xi_{i}^{\prime}=\xi_{i}, \quad \eta_{i}^{\prime}=\eta_{i}, \quad i \neq j ; \\
\lambda_{1}=\lambda_{s}, \quad \lambda_{2}=\bar{\lambda}_{s}^{-1}, \quad \mu_{s}=\lambda_{s}^{2} .
\end{gathered}
$$

Condition $\mathrm{J}$ on $Q_{\gamma_{s}}$ is equivalent to $\gamma_{s} \neq 1 / 2$. By a permutation of coordinates that preserves $\rho$, we obtain a unique holomorphic invariant $\mu_{s}$ satisfying

$$
\left|\mu_{s}\right| \geq 1, \quad \operatorname{Im} \mu_{s} \geq 0, \quad \mu_{s} \neq 0,-1, \quad 0 \leq \arg \lambda_{s} \leq \pi / 2, \quad \lambda_{s} \neq 0, i .
$$

Note that $\operatorname{Re} \gamma_{s}<1 / 2$ implies that $\left|\mu_{s}\right| \neq 1$.

For later purpose, we summarize some facts for complex type in the following.

Proposition 2.10. Let $Q_{\gamma_{s}} \subset \mathbf{C}^{4}$ be the quadric defined by (2.18) and (2.19) with $\gamma_{s} \neq 0,1$. Then $\pi_{1}$ admits two deck transformations $\tau_{11}, \tau_{12}$ such that the set of fixed points of each $\tau_{1 j}$ has dimension 3. Also, $\tau_{2 j}=\rho \tau_{1 j} \rho$ are the deck transformations of $\pi_{2}$ and

$$
\tau_{11} \tau_{21}=\tau_{21} \tau_{11}, \quad \tau_{12} \tau_{22}=\tau_{22} \tau_{12} .
$$

Let $\sigma_{s 1}=\tau_{11} \tau_{22}, \sigma_{s 2}=\tau_{12} \tau_{21}, \tau_{i}=\tau_{i 1} \tau_{i 2}$, and $\sigma_{s}=\tau_{1} \tau_{2}$. Then

$$
\sigma=\sigma_{s 1} \sigma_{s 2}=\sigma_{s 2} \sigma_{s 1}, \quad \sigma_{s 2}^{-1}=\rho \sigma_{s 1} \rho, \quad \sigma_{s}^{-1}=\rho \sigma_{s} \rho .
$$

Assume further that $\gamma_{s} \neq 1 / 2$. In suitable coordinates $\sigma_{s 1}, \sigma_{s 2}, \sigma, \rho_{s}$ are given by (2.27)(2.28), while $\sigma_{s}$ has 4 (possibly repeated) eigenvalues given by (2.25) $-(2.26)$. The $\sigma$ has four distinct eigenvalues if and only if $Q_{\gamma_{s}}$ can be holomorphically normalized so that $\operatorname{Im} \gamma_{s}>0$ and $\operatorname{Re} \gamma_{s}<1 / 2$.

In summary, we have found linear coordinates for the product quadrics such that the normal forms of $S, T_{i j}, \rho$ of the corresponding $\sigma, \sigma_{j}, \tau_{i j}, \rho_{0}$ are given by

$$
\begin{aligned}
& S: \xi_{j}^{\prime}=\mu_{j} \xi_{j}, \quad \eta_{j}^{\prime}=\mu_{j}^{-1} \eta_{j} \\
& S_{j}: \xi_{j}^{\prime}=\mu_{j} \xi_{j}, \quad \eta_{j}^{\prime}=\mu_{j}^{-1} \eta_{j}, \quad \xi_{k}^{\prime}=\xi_{k}, \quad \eta_{k}^{\prime}=\eta_{k}, \quad k \neq j ; \\
& T_{i j}: \xi_{j}^{\prime}=\lambda_{i j} \eta_{j}, \quad \eta_{j}^{\prime}=\lambda_{i j}^{-1} \xi_{j}, \quad \xi_{k}^{\prime}=\xi_{k}, \quad \eta_{k}^{\prime}=\eta_{k}, \quad k \neq j ; \\
& \rho:\left\{\begin{array}{l}
\left(\xi_{e}^{\prime}, \eta_{e}^{\prime}, \xi_{h}^{\prime}, \eta_{h}^{\prime}\right)=\left(\bar{\eta}_{e}, \bar{\xi}_{e}, \bar{\xi}_{h}, \bar{\eta}_{h}\right), \\
\left(\xi_{s}^{\prime}, \xi_{s+s_{*}}^{\prime}, \eta_{s}^{\prime}, \eta_{s+s_{*}}^{\prime}\right)=\left(\bar{\xi}_{s+s_{*}}, \bar{\xi}_{s}, \bar{\eta}_{s+s_{*}}, \bar{\eta}_{s}\right) .
\end{array}\right.
\end{aligned}
$$


Throughout the paper, the indices $h, e, s$ have the ranges $1 \leq e \leq e_{*}, e_{*}<h \leq e_{*}+h_{*}$, and $e_{*}+h_{*}<s \leq p-s_{*}$. Notice that we can always normalize $\rho_{0}$ into the above normal form $\rho$.

The commutativity of $\sigma_{h}, \sigma_{e}, \sigma_{s 1}, \sigma_{s 2}$ will be important to understand the convergence of normalization for the abelian CR singularity.

\section{Formal DECK TRANSFORMATIONS AND CENTRALIZERS}

In section 2 we show the equivalence of the classification of real analytic submanifolds $M$ that admit the maximum number of deck transformations and the classification of the families of involutions $\left\{\tau_{11}, \ldots, \tau_{1 p}, \rho\right\}$ that satisfy some mild conditions (see Proposition 2.8 ). As a consequence we show that a real submanifold is formally equivalent to a quadric if and only if its family of Moser-Webster involutions is formally linearizable.

3.1. Formal submanifolds. We first need some notation. Let $I$ be an ideal of the ring $\mathbf{R}[[x]]$ of formal power series in $x=\left(x_{1}, \ldots, x_{N}\right)$. Since $\mathbf{R}[[x]]$ is noetherian, then $I$ and its radical $\sqrt{I}$ are finitely generated. We say that $I$ defines a formal submanifold $M$ of dimension $N-k$ if $\sqrt{I}$ is generated by $r_{1}, \ldots, r_{k}$ such that at the origin all $r_{j}$ vanish and $d r_{1}, \ldots, d r_{k}$ are linearly independent. For such an $M$, let $I(M)$ denote $\sqrt{I}$ and let $T_{0} M$ be defined by $d r_{1}(0)=\cdots=d r_{k}(0)=0$. If $F=\left(f_{1}, \ldots, f_{N}\right)$ is a formal mapping with $f_{j} \in \mathbf{R}[[x]]$, we say that its set of (formal) fixed points is a submanifold if the ideal generated by $f_{1}(x)-x_{1}, \ldots, f_{N}(x)-x_{N}$ defines a submanifold. Let $I, \tilde{I}$ be ideals of $\mathbf{R}[[x]], \mathbf{R}[[y]]$ and

let $\sqrt{I}, \sqrt{\tilde{I}}$ define two formal submanifolds $M, \tilde{M}$, respectively. We say that a formal map $y=G(x)$ maps $M$ into $\widetilde{M}$ if $\tilde{I} \circ G \subset \sqrt{I}$. If $M, \tilde{M}$ are in the same space, we write $M \subset \tilde{M}$ if $\tilde{I} \subset \sqrt{I}$. We say that a formal map $F$ fixes $M$ pointwise if $I(M)$ contain each component of the mapping $F-\mathrm{I}$.

3.2. Formal deck transformations. Consider a formal $(2 p)$-submanifold in $\mathbf{C}^{2 p}$ defined by

$$
M: z_{p+j}=E_{j}\left(z^{\prime}, \bar{z}^{\prime}\right), \quad 1 \leq j \leq p .
$$

Here $E_{j}$ are formal power series in $z^{\prime}, \bar{z}^{\prime}$. We assume that

$$
E_{j}\left(z^{\prime}, \bar{z}^{\prime}\right)=h_{j}\left(z^{\prime}, \bar{z}^{\prime}\right)+q_{j}\left(\bar{z}^{\prime}\right)+O\left(\left(\left|\left(z^{\prime}, \bar{z}^{\prime}\right)\right|^{3}\right)\right.
$$

and $h_{j}, q_{j}$ are homogeneous quadratic polynomials. The formal complexification of $M$ is defined by

$$
\left\{\begin{array}{l}
z_{p+i}=E_{i}\left(z^{\prime}, w^{\prime}\right), \quad i=1, \ldots, p, \\
w_{p+i}=\bar{E}_{i}\left(w^{\prime}, z^{\prime}\right), \quad i=1, \ldots, p .
\end{array}\right.
$$

We define a formal deck transformation of $\pi_{1}$ to be a formal biholomorphic map

$$
\tau:\left(z^{\prime}, w^{\prime}\right) \rightarrow\left(z^{\prime}, f\left(z^{\prime}, w^{\prime}\right)\right), \quad \tau(0)=0
$$

such that $\pi_{1} \tau=\pi_{1}$, i.e. $E \circ \tau=E$. Recall that condition $\mathrm{B}$ says that $q_{*}=\operatorname{dim}\left\{z^{\prime} \in\right.$ $\left.\mathbf{C}^{n}: q\left(z^{\prime}\right)=0\right\}$ is zero, i.e. $q$ vanishes only at the origin in $\mathbf{C}^{p}$. 
Lemma 3.1. Let $M$ be a formal submanifold defined by (3.1)-(3.2). Suppose that $M$ satisfies condition $\mathrm{B}$. Then formal deck transformations of $\pi_{1}$ are commutative involutions. Each formal deck transformation $\tau$ of $\pi_{1}: \mathcal{M} \rightarrow \mathbf{C}^{p}$ is uniquely determined by its linear part $L \tau$ in the $\left(z^{\prime}, w^{\prime}\right)$ coordinates, while $L \tau$ is a deck transformation for the complexification for $\pi_{1}: \mathcal{Q} \rightarrow \mathbf{C}^{p}$, where $\mathcal{Q}$ is the complexification of the quadratic part $Q$ of $M$. If $M$ is real analytic, all formal deck transformations of $\pi_{1}$ are convergent.

Proof. Let us recall some results about the quadric $Q$. We already know that $q_{*}=0$ implies that $\pi_{1}$ for the complexification of $Q$ is a branched covering. As used in the proof of Lemma 2.1, $\pi_{1}$ is an open mapping near the origin and its regular values are dense. In particular, we have

$$
\operatorname{det} \partial_{w^{\prime}}\left\{h\left(z^{\prime}, w^{\prime}\right)+q\left(w^{\prime}\right)\right\} \not \equiv 0
$$

Let $\tau$ be a formal deck transformation for $M$. To show that $\tau$ is an involution, we note that its linear part at the origin, $L \tau$, is a deck transformation of $Q$. Hence $L \tau$ is an involution. Replacing $\tau$ by the deck transformation $\tau^{2}$, we may assume that $\tau$ is tangent to the identity. Write

$$
\tau\left(z^{\prime}, w^{\prime}\right)=\left(z^{\prime}, w^{\prime}+u\left(z^{\prime}, w^{\prime}\right)\right) .
$$

We want to show that $u=0$. Assume that $u\left(z^{\prime}, w^{\prime}\right)=O\left(\left|\left(z^{\prime}, w^{\prime}\right)\right|^{k}\right)$ and let $u_{k}$ be homogeneous and of degree $k$ such that $u\left(z^{\prime}, w^{\prime}\right)=u_{k}\left(z^{\prime}, w^{\prime}\right)+O\left(\left|\left(z^{\prime}, w^{\prime}\right)\right|^{k+1}\right)$. We have

$$
E\left(z^{\prime}, w^{\prime}+u\left(z^{\prime}, w^{\prime}\right)\right)=E\left(z^{\prime}, w^{\prime}\right) .
$$

Comparing terms of order $k+1$, we get

$$
\partial_{w^{\prime}}\left\{h\left(z^{\prime}, w^{\prime}\right)+q\left(w^{\prime}\right)\right\} u_{k}\left(z^{\prime}, w^{\prime}\right)=0 .
$$

By (3.3),$u_{k}=0$. This shows that each formal deck transformation $\tau$ of $\pi_{1}$ for $M$ is an involution. As mentioned above, $L \tau$ is a deck transformation of $\pi_{1}$ for $Q$. Also if $\tau, \tilde{\tau}$ are commuting formal involutions then $\tau^{-1} \tilde{\tau}$ is an involution and $\tau=\tilde{\tau}$ if and only if $L \tau=L \tilde{\tau}$.

Assume now that $M$ is real analytic. We want to show that each formal deck transformation $\tau$ is convergent. By a theorem of Artin Art68 applied to the solution $u$, there is a convergent $\tilde{\tau}\left(z^{\prime}, w^{\prime}\right)=\tau\left(z^{\prime}, w^{\prime}\right)+O\left(\left|\left(z^{\prime}, w^{\prime}\right)\right|^{2}\right)$ such that $E \circ \tilde{\tau}=E$, i.e. $\tilde{\tau}$ is a deck transformation. Then $\tilde{\tau}^{-1} \tau$ is a deck transformation tangent to the identity. Since it is a formal involution by the above argument, then it must be identity. Therefore, $\tau=\tilde{\tau}$ converges.

Analogous to real analytic submanifolds, we say that a formal manifold defined by (3.1)(3.2) satisfies condition $\mathrm{D}$ if its formal branched covering $\pi_{1}$ admits $2^{p}$ formal deck transformations.

Recall from section 2 that it is crucial to distinguish a special set of generators for the deck transformations in order to relate the classification of real analytic manifolds to the classification of certain $\left\{\tau_{11}, \ldots, \tau_{1 p}, \rho\right\}$. The set of generators is uniquely determined by the dimension of fixed-point sets. We want to extend these results at the formal level.

Proposition 3.2. Let $M, \tilde{M}$ be formal p-submanifolds in $\mathbf{C}^{n}$ of the form (3.1)-(3.2). Suppose that $M, \tilde{M}$ satisfy condition D. Then the following hold:

(i) $M$ and $\tilde{M}$ are formally equivalent if and only if their associated families of involutions $\left\{\tau_{11}, \ldots, \tau_{1 p}, \rho\right\}$ and $\left\{\tilde{\tau}_{11}, \ldots, \tilde{\tau}_{1 p}, \rho\right\}$ are formally equivalent. 
(ii) Let $\mathcal{T}_{1}=\left\{\tau_{11}, \ldots, \tau_{1 p}\right\}$ be a family of formal holomorphic involutions which commute pairwise. Suppose that the tangent spaces of $\operatorname{Fix}\left(\tau_{11}\right), \ldots, \operatorname{Fix}\left(\tau_{1 p}\right)$ are hyperplanes intersecting transversally at the origin. Let $\rho$ be an anti-holomorphic formal involution and let $\mathcal{T}_{2}=\left\{\tau_{21}, \ldots, \tau_{2 p}\right\}$ with $\tau_{2 j}=\rho \tau_{1 j} \rho$. Suppose that $\sigma=\tau_{1} \tau_{2}$ has distinct eigenvalues for $\tau_{i}=\tau_{i 1} \cdots \tau_{i p}$, and

$$
\left[\mathfrak{M}_{n}\right]_{1}^{L \mathcal{T}_{1}} \cap\left[\mathfrak{M}_{n}\right]_{1}^{L \mathcal{T}_{2}}=\{0\}
$$

There exists a formal submanifold defined by

$$
z^{\prime \prime}=\left(B_{1}^{2}, \ldots, B_{p}^{2}\right)\left(z^{\prime}, \bar{z}^{\prime}\right)
$$

for some formal power series $B_{1}, \ldots, B_{p}$ such that $M$ satisfies condition $\mathrm{D}$. The set of involutions $\left\{\tilde{\tau}_{11}, \ldots, \tilde{\tau}_{1 p}, \rho_{0}\right\}$ of $M$ is formally equivalent to $\left\{\tau_{11}, \ldots, \tau_{1 p}, \rho\right\}$.

Proof. (i) Let $M$ and $\tilde{M}$ be given by $z^{\prime \prime}=E\left(z^{\prime}, \overline{z^{\prime}}\right)$ and $\tilde{z}^{\prime \prime}=\tilde{E}\left(\tilde{z}^{\prime}, \overline{\tilde{z}^{\prime}}\right)$, respectively. Suppose that $f$ is a formal holomorphic transformation sending $M$ into $\tilde{M}$. We have

$$
f^{\prime \prime}\left(z^{\prime}, E\left(z^{\prime}, w^{\prime}\right)\right)=\tilde{E}\left(f^{\prime}\left(z^{\prime}, E\left(z^{\prime}, w^{\prime}\right)\right), \bar{f}^{\prime}\left(w^{\prime}, \bar{E}\left(w^{\prime}, z^{\prime}\right)\right)\right) .
$$

Here $f=\left(f^{\prime}, f^{\prime \prime}\right)$. Recall that $\rho_{0}\left(z^{\prime}, w^{\prime}\right)=\left(\overline{w^{\prime}}, \overline{z^{\prime}}\right)$. Define a formal mapping $\left(z^{\prime}, w^{\prime}\right) \rightarrow$ $\left(\tilde{z}^{\prime}, \tilde{w}^{\prime}\right)=F\left(z^{\prime}, w^{\prime}\right)$ by

$$
F\left(z^{\prime}, w^{\prime}\right):=\left(f^{\prime}\left(z^{\prime}, E\left(z^{\prime}, w^{\prime}\right)\right), \overline{f^{\prime}}\left(w^{\prime}, \bar{E}\left(w^{\prime}, z^{\prime}\right)\right)\right) .
$$

It is clear that $F \rho_{0}=\rho_{0} F$. By Lemma 2.5, we know that $\tilde{z}^{\prime}$ and $\tilde{z}^{\prime \prime}=\tilde{E}\left(\tilde{z}^{\prime}, \tilde{w}^{\prime}\right)$ generate invariant formal power series of $\left\{\tilde{\tau}_{1 j}\right\}$. Thus, $\tilde{z}^{\prime} \circ F\left(z^{\prime}, w^{\prime}\right)=f^{\prime}\left(z^{\prime}, E\left(z^{\prime}, w^{\prime}\right)\right)$ and $\tilde{E} \circ F\left(z^{\prime}, w^{\prime}\right)$ are invariant by $F^{-1} \circ \tilde{\tau}_{1 j} \circ F$. By (3.5) and the definition of $F$,

$$
\tilde{E} \circ F\left(z^{\prime}, w^{\prime}\right)=f^{\prime \prime}\left(z^{\prime}, E\left(z^{\prime}, w^{\prime}\right)\right) \text {. }
$$

This shows that $f\left(z^{\prime}, E\left(z^{\prime}, w^{\prime}\right)\right)$ is invariant under $F^{-1} \circ \tilde{\tau}_{1 j} \circ F$. Since $f$ is invertible, then $z^{\prime}$ and $E\left(z^{\prime}, w^{\prime}\right)$ are invariant under $F^{-1} \circ \tilde{\tau}_{1 j} \circ F$. Therefore, $\left\{\tau_{1 j}\right\}$ and $\left\{F^{-1} \circ \tilde{\tau}_{1 i} \circ F\right\}$ are the same by Lemma 2.5 as they have the same invariant functions.

Assume now that $\left\{\tau_{1 j}\right\}=\left\{F^{-1} \circ \tilde{\tau}_{1 i} \circ F\right\}$ for some formal biholomorphic map $F$ commuting with $\rho_{0}$. Recall that $\tilde{z}^{\prime}, \tilde{z}^{\prime \prime}$ are invariant by $\tilde{\tau}_{1 j}$. Then $\tilde{z}^{\prime} \circ F$ and $\tilde{E} \circ F$ are invariant by $\left\{\tau_{1 j}\right\}$. By Lemma 2.5, invariant power series of $\tau_{1 j}$ are generated by $z^{\prime}, E\left(z^{\prime}, w^{\prime}\right)$. Thus we can write

$$
\begin{aligned}
& \tilde{z}^{\prime} \circ F\left(z^{\prime}, w^{\prime}\right)=f^{\prime}\left(z^{\prime}, E\left(z^{\prime}, w^{\prime}\right)\right), \\
& \tilde{E} \circ F\left(z^{\prime}, w^{\prime}\right)=f^{\prime \prime}\left(z^{\prime}, E\left(z^{\prime}, w^{\prime}\right)\right)
\end{aligned}
$$

for some formal power series map $f=\left(f^{\prime}, f^{\prime \prime}\right)$. Since $\rho_{0} F=F \rho_{0}$, then by (3.6)

$$
F\left(z^{\prime}, w^{\prime}\right)=\left(f^{\prime}\left(z^{\prime}, 0\right), \bar{f}^{\prime}\left(w^{\prime}, 0\right)\right)+O\left(\left|\left(z^{\prime}, w^{\prime}\right)\right|^{2}\right) .
$$

Since $F$ is (formally) biholomorphic then $z^{\prime} \rightarrow f^{\prime}\left(z^{\prime}, 0\right)$ is biholomorphic. Then

$$
f^{\prime \prime}\left(0, E\left(0, w^{\prime}\right)\right)=\tilde{E}\left(0, \bar{f}^{\prime}\left(w^{\prime}, 0\right)\right)+O\left(\left|w^{\prime}\right|^{3}\right) \text {. }
$$

We have $E\left(0, w^{\prime}\right)=q\left(w^{\prime}\right)+O\left(\left|w^{\prime}\right|^{3}\right)$ and $\tilde{E}\left(0, w^{\prime}\right)=\tilde{q}\left(w^{\prime}\right)+O\left(\left|w^{\prime}\right|^{3}\right)$. Here $q\left(w^{\prime}\right), \tilde{q}\left(w^{\prime}\right)$ are quadratic. By condition $q_{*}=0$, we know that $\tilde{q}_{1}, \ldots, \tilde{q}_{p}$ and hence $\tilde{q}_{1} \circ L, \ldots, \tilde{q}_{p} \circ L$ are linearly independent. Here $L$ is the linear part of the mapping $w^{\prime} \rightarrow \bar{f}^{\prime}\left(w^{\prime}, 0\right)$, which is invertible. This shows that the linear part of $w^{\prime} \rightarrow f^{\prime \prime}\left(0, w^{\prime}\right)$ is biholomorphic. By (3.7), 
$f^{\prime \prime}\left(z^{\prime}, 0\right)=O\left(\left|z^{\prime}\right|^{2}\right)$. Hence $f=\left(f^{\prime}, f^{\prime \prime}\right)$ is biholomorphic. By a simple computation, we have $f(M)=\tilde{M}$, i.e.

for $z^{\prime \prime}=E\left(z^{\prime}, \bar{z}^{\prime}\right)$.

$$
\tilde{E}\left(f^{\prime}(z), \overline{f^{\prime}(z)}\right)=f^{\prime \prime}(z)
$$

(ii) Assume that $\left\{\tau_{1 j}\right\}$ and $\rho$ are given in the $(\xi, \eta)$ space. We want to show that a formal holomorphic equivalence class of $\left\{\tau_{1 j}, \rho\right\}$ can be realized by a formal submanifold satisfying condition D. The proof is almost identical to the realization proof of Proposition 2.8 and we will be brief. Using a formal, instead of convergent, change of coordinates, we know that invariant formal power series of $\left\{\tau_{1 j}\right\}$ are generated by

$$
z^{\prime}=\left(A_{1}(\xi, \eta), \ldots, A_{p}(\xi, \eta)\right), \quad z^{\prime \prime}=\left(B_{1}^{2}(\xi, \eta), \ldots, B_{p}^{2}(\xi, \eta)\right),
$$

where $B_{j}$ is skew-invariant by $\tau_{1 j}$, and $A, B_{i}$ are invariant under $\tau_{1 j}$ for $i \neq j$. Moreover, $\phi(\xi, \eta)=(A, B)(\xi, \eta)$ is formally biholomorphic. Set

$$
w_{j}^{\prime}=\overline{A_{j} \circ \rho(\xi, \eta)}, \quad w_{j}^{\prime \prime}=\overline{B_{j}^{2} \circ \rho(\xi, \eta)} .
$$

Then $(\xi, \eta) \rightarrow(A(\xi, \eta), \overline{A \circ \rho(\xi, \eta)})$ has an inverse $\psi$. Define

$$
M: z^{\prime \prime}=\left(B_{1}^{2}, \ldots, B_{p}^{2}\right) \circ \psi\left(z^{\prime}, \bar{z}^{\prime}\right) .
$$

The complexification of $M$ is given by

$$
\mathcal{M}: z^{\prime \prime}=\left(B_{1}^{2}, \ldots, B_{p}^{2}\right) \circ \psi\left(z^{\prime}, w^{\prime}\right), \quad w^{\prime \prime}=\left(\bar{B}_{1}^{2}, \ldots, \bar{B}_{p}^{2}\right) \circ \bar{\psi}\left(w^{\prime}, z^{\prime}\right) .
$$

Note that $\phi \circ \psi\left(z^{\prime}, w^{\prime}\right)=\left(z^{\prime}, B \circ \psi\left(z^{\prime}, w^{\prime}\right)\right)$. Since $\phi \psi$ is invertible, the linear part $D$ of $B \circ \psi$ satisfies $\left|D\left(0, w^{\prime}\right)\right| \geq\left|w^{\prime}\right| / C$. This shows that $q_{*}=0$. As in the proof of Proposition 2.8, we can verify that $M$ is a realization for $\left\{\tau_{1 j}, \rho\right\}$.

\section{Normal FORMS OF COMMUTING BIHOLOMORPHiSMS}

In this section, we shall consider a family of commuting germs of holomorphic diffeomorphisms at a common fixed point, say $0 \in \mathbf{C}^{n}$. We shall give conditions that ensure that the family can be transformed simultaneously and holomorphically to a normal form. This means that there exists a germ of biholomorphism at the origin which conjugates each germ of biholomorphism in the family to a mapping that commutes with the linear part of every mapping of the family.

\subsection{Centralizers and Decomposition.}

Definition 4.1. Let $\mathcal{F}$ be a family of formal mappings on $\mathbf{C}^{n}$ fixing the origin. Let $\mathcal{C}(\mathcal{F})$ be the centralizer of $\mathcal{F}$, i.e. the set of formal holomorphic mappings $g$ that fix the origin and commute with each element $f$ of $\mathcal{F}$, i.e., $f \circ g=g \circ f$.

Let $\mathcal{C}_{2}(\mathcal{F})$ be the "higher order formal centralizer" of $\mathcal{F}$, that is

$$
\mathcal{C}_{2}(\mathcal{F})=\left\{H \in\left(\widehat{\mathfrak{M}}_{n}^{2}\right)^{n} \mid H \circ F=F \circ H, F \in \mathcal{F}\right\} .
$$

We now deal with the following decomposition problem: Let $\mathcal{C}$ be a set of analytic mappings. We want to decompose an arbitrary invertible mapping into the composition of an element of a centralizer of $\mathcal{C}$ and an element which is normalized with respect to $\mathcal{C}$. We first prove a general convergence decomposition, which will be used several times. Let $e_{j}$ denote the standard $j$ th unit vector of $\mathbf{C}^{n}$. 
Definition 4.2. Let $\mathcal{A}$ be a group of permutations of $\{1, \ldots, n\}$. Then $\mathcal{A}$ acts on the right (resp. on the left) on $\widehat{\mathcal{O}}_{n}^{n}$ by permutation of variables $z=\left(z_{1}, \ldots, z_{n}\right)$ as follows: Let $F(z)=\sum_{|Q|>0} F_{Q} z^{Q}$ be a formal mapping from $\mathbf{C}^{n}$ to $\mathbf{C}^{n}$, and let $\nu, \mu \in \mathcal{A}$; set

$$
\nu \circ F \circ \mu(z):=\sum_{i} \sum_{Q \in \mathbf{N}^{n}} F_{\nu(i), \mu^{-1}(Q)} z^{Q} e_{i} .
$$

Define the components $(\mathcal{A} F)_{i},(F \mathcal{A})_{i}$, and consequently $(\mathcal{A F} \mathcal{A})_{i}$ by

$$
\begin{aligned}
(\mathcal{A} F)_{i}(z) & :=\sum_{Q \in \mathbf{N}^{n}} \max _{\nu \in \mathcal{A}}\left|F_{\nu(i), Q}\right| z^{Q}, \quad(F \mathcal{A})_{i}(z):=\sum_{Q \in \mathbf{N}^{n}} \max _{\mu \in \mathcal{A}}\left|F_{i, \mu^{-1}(Q)}\right| z^{Q}, \\
(\mathcal{A} F \mathcal{A})_{i}(z) & =\sum_{Q \in \mathbf{N}^{n}} \max _{(\nu, \mu) \in \mathcal{A}^{2}}\left|F_{\nu(i), \mu^{-1}(Q)}\right| z^{Q} .
\end{aligned}
$$

We see that $F \mathcal{A}$ is the smallest (w.r.t. $\prec$ ) power series mapping that majorizes $F$ and is right-invariant under $\mathcal{A}$, while $\mathcal{A} F$ is the smallest power series mapping that majorizes $F$ and is left-invariant under $\mathcal{A}$. In particular, if $F, G$ are mappings without constant or linear terms, then

$$
\mathcal{A}(F \circ(I+G)) \mathcal{A} \prec(\mathcal{A} F \mathcal{A})(\mathcal{A} I \mathcal{A}+\mathcal{A} G \mathcal{A})
$$

where the last relation holds if the composition is well-defined.

To simply our notation, we will take $\mathcal{A}$ to be the full permutation group of $\{1, \ldots, n\}$. We will denote

$$
F_{\text {sym }}=\mathcal{A} F \mathcal{A} \text {. }
$$

Lemma 4.3. Let $\hat{\mathcal{H}}$ be a real subspace of $\left(\widehat{\mathfrak{M}}_{n}^{2}\right)^{n}$. Let $\pi:\left(\widehat{\mathfrak{M}}_{n}^{2}\right)^{n} \rightarrow \hat{\mathcal{H}}$ be a $\mathbf{R}$ linear projection $\left(\right.$ i.e. $\pi^{2}=\pi$ ) that preserves the degrees of the mappings and let $\hat{\mathcal{G}}:=(\mathrm{I}-\pi)\left(\widehat{\mathfrak{M}}_{n}^{2}\right)^{n}$. Suppose that there is a positive constant $C$ such that

$$
\pi(E) \prec C E_{\text {sym }}
$$

for any $E \in\left(\widehat{\mathfrak{M}}_{n}^{2}\right)^{n}$. Let $F$ be a formal map tangent to the identity. There exists a unique decomposition

$$
F=H G^{-1}
$$

with $G-I \in \hat{\mathcal{G}}$ and $H-I \in \hat{\mathcal{H}}$. If $F$ is convergent, then $G$ and $H$ are also convergent.

Proof. If $f$ is a formal mapping, we define the $k$-jet:

$$
J^{k} f(z)=\sum_{|Q| \leq k} f_{Q} z^{Q} .
$$

Write $F=I+f, G=I+g$ and $H=I+h$. We need to solve $F G=H$, i.e to solve

$$
h-g=f(I+g) \text {. }
$$

Since $f^{\prime}(0)=0$, then for any $k \geq 2$, the $k$-jet of $f(I+g)$ depends only on the $(k-1)$-jet of $g$. Since $\pi$ is linear and preserves degrees, (4.2) implies that $J^{k}$ commutes with $\pi$. Hence we can define, for all $k \geq 2$,

$$
-J^{k}(g):=\pi\left(J^{k}(f(I+g)), \quad J^{k}(h):=(I-\pi)\left(J^{k}(f(I+g)) .\right.\right.
$$


This solves the formal decomposition uniquely. Assume that $F$ is a germ of holomorphic mapping. Hence, we have

$$
g \prec C(f(I+g))_{\text {sym }} \prec C f_{\text {sym }}\left(I_{\text {sym }}+g_{\text {sym }}\right) .
$$

Since $g_{\text {sym }}$ is the smallest left and right $\mathcal{A}$ invariant power series that dominates $g$, we have

$$
g_{\text {sym }} \prec C f_{\text {sym }}\left(I_{\text {sym }}+g_{\text {sym }}\right) .
$$

Therefore, $g_{\text {sym }}$ is dominated by the solution $u$ to

$$
u=C f_{\text {sym }}\left(I_{\text {sym }}+u\right), \quad u(0)=0 .
$$

Notice that $u$ is real analytic near the origin by the implicit function theorem. So, $g_{\text {sym }}$ is convergent, and $g, h=g+f(I+g)$ are convergent.

Remark 4.4. Let $\mathcal{A}, \mathcal{B}$ be two subgroups of permutations. Instead of using the full permutations group, we could have used $G_{\text {sym }}:=\mathcal{A} G \mathcal{B}$. We have

$$
G \prec \mathcal{A} G \mathcal{B} \prec C \mathcal{A}(F \circ(I+G)) \mathcal{B} \prec(\mathcal{A} F \mathcal{A})(\mathcal{A I B}+\mathcal{A} G \mathcal{B})
$$

4.2. Abelian family of biholomorphisms. Let $\mathbf{D}_{i}:=\operatorname{diag}\left(\mu_{i 1}, \ldots, \mu_{i n}\right)$ with $1 \leq i \leq \ell$ be diagonal invertible matrices of $\mathbf{C}^{n}$. Let $D_{i}: x \rightarrow \mathbf{D}_{i} x$ be the linear mappings. Let $D$ denote the family $\left\{D_{i}\right\}_{i=1, \ldots \ell}$ of linear mappings.

Definition 4.5. We say that $F=\left(f_{1}, \ldots, f_{n}\right)$ is normalized with respect to $D$ if it is tangent to the identity and it does not have components along the centralizer of $D$, i.e. for each $Q$ with $|Q| \geq 2$,

$$
f_{j, Q}=0, \quad \text { if } \mu_{i}^{Q}=\mu_{i j} \text { for all } i .
$$

Let $\mathcal{C}^{\mathrm{c}}(D)$ denote the set of formal mappings normalized with respect to $D$. Let $\mathcal{C}_{2}^{\mathrm{c}}(D)$ be the set of all $H \in\left(\widehat{\mathfrak{M}}_{n}^{2}\right)^{n}$ satisfying $I+H \in \mathcal{C}^{\mathrm{c}}(D)$.

Let us consider a family $F:=\left\{F_{i}\right\}_{i=1}^{\ell}$ of germs of holomorphic diffeomorphisms of $\left(\mathbf{C}^{n}, 0\right)$ of which the linear of $F_{i}(x)$ at the origin is $D_{i}$. Thus

$$
F_{i}(x)=\mathbf{D}_{i} x+f_{i}(x), \quad f_{i}(0)=0, \quad D f_{i}(0)=0 .
$$

The group of germs of (resp. formal) biholomorphisms tangent to identity acts on the family $F$ by $\Phi_{*} F:=\left\{\Phi^{-1} \circ F_{i} \circ \Phi: 1 \leq i \leq \ell\right\}$.

Let $\left\{F_{i}\right\}_{i=1, \ldots \ell}$ be a family of commuting germs of biholomorphisms with $\ell<\infty$. Let us recall a result by M. Chaperon (see theorem 4 in [Cha86], page 132):

Proposition 4.6. If the family of diffeomorphisms is abelian then there exists a formal diffeomorphism $\Phi$, which is tangent to the identity, such that

$$
\widehat{F}_{i}\left(\mathbf{D}_{j} x\right)=\mathbf{D}_{j} \widehat{F}_{i}(x), \quad 1 \leq i, j \leq \ell
$$

where $\widehat{F}_{i}:=\Phi_{*} F_{i}$, for $1 \leq i \leq \ell$. We call the family $\left\{\widehat{F}_{i}\right\}$ a formal normal form of the family $F$ (or a normalized family) with respect to the family $D$ of linear maps.

For convenience, we restrict changes of holomorphic coordinates to the ones that are tangent to the identity. Also $\Phi_{*}\left\{F_{i}\right\}_{i=1}^{\ell}=\left\{\tilde{F}_{i}\right\}_{i=1}^{\ell}$ means that

$$
\Phi_{*} F_{i}=\tilde{F}_{i}, \quad 1 \leq i \leq \ell
$$


These restrictions will be removed by mild changes. For instance, if $\Phi$ transforms a family $F$ into a family $\hat{F}$ that commutes with $L F$, the family of the linear part of the $F$, then $(L \Phi)^{-1}\left(L F_{i}\right) L \Phi=L \hat{F}_{i}$. Therefore, $\Phi(L \Phi)^{-1}$ is tangent to the identity and transforms $F$ into $(L \Phi) \hat{F}(L \Phi)^{-1}$ which commutes with $L F$.

Let $\widehat{\mathcal{O}}_{n}^{D}$ be the ring of formal invariants of the family $D$, that is

$$
\widehat{\mathcal{O}}_{n}^{D}:=\left\{f \in \widehat{\mathcal{O}}_{n} \mid f\left(\mathbf{D}_{i} x\right)=f(x), i=1, \ldots, \ell\right\} .
$$

If $Q \in \mathbf{N}^{n}, Q \neq 0$, then $x^{Q} \in \widehat{\mathcal{O}}_{n}^{D}$ if and only if

$$
\mu_{i}^{Q}:=\mu_{i 1}^{q_{1}} \cdots \mu_{i n}^{q_{n}}=1, \quad \forall 1 \leq i \leq \ell .
$$

If $|Q|>1$, then $x^{Q} e_{j} \in \mathcal{C}_{2}(D)$ if and only if $\mu_{i}^{Q}=\mu_{i j}, \forall 1 \leq i \leq \ell$. With notation of Definition 4.1, we have

Lemma 4.7. Any formal diffeomorphism $\Phi$ of $\left(\mathbf{C}^{n}, 0\right)$, tangent to identity, can be written uniquely as $\Phi=\Phi_{1} \circ \Phi_{0}^{-1}$ with $\Phi_{1} \in \mathcal{C}^{\mathrm{c}}(D)$ and $\Phi_{0} \in \mathcal{C}(D)$. Furthermore, $\Phi_{0}, \Phi_{1}$ are convergent when $\Phi$ is convergent.

Proof. This follows from Lemma 4.3, where $\hat{\mathcal{H}}$ is replaced by $\mathcal{C}_{2}(D)$ and $\pi$ is defined by

$$
\pi\left(\sum f_{j, Q} x^{Q} e_{j}\right)=\sum_{j} \sum_{x^{Q} e_{j} \notin \mathcal{C}_{2}(D)} f_{j, Q} x^{Q} e_{j} .
$$

Lemma 4.8. Let $\hat{F}:=\left\{\hat{F}_{i}\right\}$ and $\tilde{F}:=\left\{\tilde{F}_{i}\right\}$ be two formal normal forms of the abelian family of diffeomorphisms $F:=\left\{F_{i}\right\}$. There exists a formal diffeomorphism $\Phi$, tangent to identity at the origin, such that $\Phi \in \mathcal{C}(D)$ and $\Phi \circ \tilde{F}_{i}=\hat{F}_{i} \circ \Phi$. Furthermore, there is a unique $\Phi \in \mathcal{C}^{c}(D)$ that transforms the family $F$ into a normal form.

Proof. Since both $\hat{F}$ and $\tilde{F}$ are normal forms of $F$, there exists a formal diffeomorphism $\Phi$, tangent to identity at the origin, such that $\tilde{F}_{i} \circ \Phi=\Phi \circ \hat{F}_{i}$. According to Lemma 4.7, we can decompose $\Phi=\Phi_{1} \circ \Phi_{0}^{-1}$ where $\Phi_{0} \in \mathcal{C}(D)$ and $\Phi_{1} \in \mathcal{C}^{\mathrm{c}}(D)$. Hence, we have $\Phi_{1}^{-1} \circ \tilde{F}_{i} \circ \Phi_{1}=\Phi_{0}^{-1} \circ \hat{F}_{i} \circ \Phi_{0}$. Let us set $G_{i}:=\Phi_{0}^{-1} \circ \hat{F}_{i} \circ \Phi_{0}$. Then $G_{i}$ is a formal diffeomorphism satisfying $G_{i}(x)-\mathbf{D}_{i} x \in \mathcal{C}(D)$. Let us show by induction on $N \geq 2$ that if $\Phi_{1}=I+\Phi_{1}^{N}+O(N+1)$ with $\Phi_{1}^{N}$ being homogeneous of degree $N$, then $\Phi_{1}^{N}=\overline{0}$. Indeed, a computation shows that

$$
\left\{G_{i}\right\}_{N}=\left\{\hat{F}_{i}\right\}_{N}+D_{i} \circ \Phi_{1}^{N}-\Phi_{1}^{N} \circ D_{i}
$$

Express $\Phi_{1}^{N}$ as sum of monomial mappings. The monomial mappings are not in $\mathcal{C}(D)$, while those of $F_{i}$ and $G_{i}$ are. We obtain $\Phi_{1}^{N}=0$.

To verify the last assertion, assume that $\Psi_{*} F=\hat{F}$ and $\tilde{\Psi}_{*} F=\tilde{F}$ are in the normal form. Suppose that $\Psi, \tilde{\Psi}$ are normalized. Then $\left(\Psi^{-1} \tilde{\Psi}\right)_{*} \hat{F}=\tilde{\Psi}_{*}\left(\Psi^{-1}\right)_{*} \hat{F}$ is in the normal form. Write $\Psi^{-1} \tilde{\Psi}=\psi_{1} \psi_{0}^{-1}$ with $\psi_{1} \in \mathcal{C}^{\mathrm{c}}(D)$ and $\psi_{0} \in \mathcal{C}(D)$. Then $\left(\psi_{1}\right)_{*} \hat{F}$ is in a normal form. From the above proof, we know that $\psi_{1}=I$. Now $\Psi=\tilde{\Psi} \psi_{0}$, which implies that $\Psi=\tilde{\Psi}$.

Lemma 4.9. If a formal normal form of $F$ is completely integrable so are all other normal forms of $F$; in particular, the unique $\Phi$ in Lemma 4.8 transforms $F$ into a completely integrable normal form. 
Proof. By Lemma 4.8, we transform a normal form $\left\{\hat{F}_{i}\right\}$ into another one $\left\{\tilde{F}_{i}\right\}$ by applying a transformation $\Phi$ that commutes with each $D_{j}$. Hence, we have $\hat{F}_{i}:=\Phi^{-1} \circ \tilde{F}_{i} \circ \Phi$, for all $i=1, \ldots, \ell$. Let us write $\Phi(x)=\sum_{Q \in \mathbf{N}^{n}, 1 \leq j \leq n} \phi_{j, Q} x^{Q} e_{j}$. Suppose that $\left\{\hat{F}_{i}\right\}$ is completely integrable, then

$$
\Phi \circ \hat{F}_{i}(x)=\sum_{Q \in \mathbf{N}^{n}} \phi_{j, Q} \mu_{i}(x)^{Q} x^{Q} e_{j} .
$$

Since $\Phi$ commutes with each $D_{j}$, then

$$
\Phi \circ \hat{F}_{i}(x)=\operatorname{diag}\left(\mu_{i 1}(x), \ldots, \mu_{i n}(x)\right) \cdot \Phi(x) .
$$

The conjugacy equation leads to

$$
\mu_{i j}(x) \cdot \Phi_{j}(x)=\tilde{F}_{i j}(\Phi(x)), \quad 1 \leq j \leq n .
$$

As a consequence, we have $\tilde{F}_{i}(x)=\operatorname{diag}\left(\left(\tilde{\mu}_{i 1}(x), \ldots, \tilde{\mu}_{i n}(x)\right) \cdot x\right.$ with $\left(\tilde{\mu}_{i j} \circ \Phi(x)\right) \cdot \Phi_{j}(x)=$ $\mu_{i j}(x) \cdot \Phi_{j}(x)$, i.e. $\tilde{\mu}_{i j}=\mu_{i j} \circ \Phi^{-1}$.

Each function $\tilde{\mu}_{i j}$ is an invariant function of $D$ since

$$
\tilde{\mu}_{i j}\left(\mathbf{D}_{k} x\right)=\mu_{i j} \circ \Phi^{-1}\left(\mathbf{D}_{k} x\right)=\mu_{i j} \circ D_{k}\left(\Phi^{-1}(x)\right)=\mu_{i j} \circ \Phi^{-1}(x) .
$$

The second and third conditions of the definition of the complete integrability is obviously satisfied by $\left\{\tilde{F}_{i}\right\}$ since $\tilde{\mu}_{i j}=\mu_{i j} \circ \Phi^{-1}$.

Lemma 4.10. If a formal normal form of $F$ is linear so are all other normal forms of $F$.

Proof. According to Lemma 4.8, we transform a linear normal form $\left\{\hat{F}_{i}\right\}$ into another one $\left\{\tilde{F}_{i}\right\}$ by applying a transformation $\Phi$ that commutes with each $D_{j}$. Since $\hat{F}_{i}(x)=\mathbf{D}_{i} x$, we have $\tilde{F}_{i}=\Phi^{-1}\left(D_{i} \Phi(x)\right)$, for all $i=1, \ldots, \ell$. Since $\Phi$ commutes with each map $x \mapsto \mathbf{D}_{i} x$, then

$$
\tilde{F}_{i}=\Phi^{-1}\left(D_{i} \Phi(x)\right)=\Phi^{-1}\left(\Phi\left(\mathbf{D}_{i} x\right)\right)=\mathbf{D}_{i} x .
$$

Definition 4.11. We say that the family $D$ is of Poincaré type if there exist constants $d>1$ and $c>0$ such that, for each $(j, Q) \in\{1, \ldots, n\} \times \mathbf{N}^{n}$ that satisfies $\mu_{m}^{Q}-\mu_{m j} \neq 0$ for some $m$, there exists $\left(i, Q^{\prime}\right) \in\{1, \ldots, \ell\} \times \mathbf{N}^{n}$ such that $\mu_{k}^{Q^{\prime}}=\mu_{k}^{Q}$ for all $1 \leq k \leq \ell$, $\mu_{i}^{Q^{\prime}}-\mu_{i j} \neq 0$, and

$$
\max \left(\left|\mu_{i}^{Q^{\prime}}\right|,\left|\mu_{i}^{-Q^{\prime}}\right|\right)>c^{-1} d^{\left|Q^{\prime}\right|}, \quad Q^{\prime}-Q \in \mathbf{N}^{n} \cup\left(-\mathbf{N}^{n}\right) .
$$

Such a condition has appeared in the definition of the good set in [BHV10].

Definition 4.12. Let $f=\sum_{Q \in \mathbf{N}^{n}} f_{Q} x^{Q}$ and $g=\sum_{Q \in \mathbf{N}^{n}} g_{Q} x^{Q}$ be two formal power series. We say that $g$ majorizes $f$, written as $f \prec g$, if $g_{Q} \geq 0$ and $\left|f_{Q}\right| \leq g_{Q}$ for all $Q \in \mathbf{N}^{n}$. Set

$$
\bar{f}:=\sum_{Q \in \mathbf{N}^{n}}\left|f_{Q}\right| x^{Q} .
$$

Theorem 4.13. Let $F$ be an abelian family of germs of holomorphic diffeomorphisms at the origin of $\mathbf{C}^{n}$. Assume that it is formally completely integrable and that its linear part at the origin is of Poincaré type. Then $F$ is holomorphically conjugated to a normal form $\hat{F}=\left\{\hat{F}_{1}, \ldots, \hat{F}_{\ell}\right\}$ so that each $\hat{F}_{i}$ is defined by

$$
x_{j}^{\prime}=\mu_{i j}(x) x_{j}, \quad j=1, \ldots, n
$$


where $\mu_{i j}(x)$ are germs of holomorphic functions invariant under $D$ and $\mu_{i j}(0)=\mu_{i j}$. In fact, the unique normalized mapping $\Phi$ in Lemma 4.8 is convergent.

The primary example is the following Moser-Webster normal form of reversible mappings:

$$
\hat{\sigma}: \xi^{\prime}=M_{1}(\xi \eta, \zeta) \xi \quad \eta^{\prime}=M_{1}^{-1}(\xi \eta, \zeta) \eta, \quad \zeta^{\prime}=\zeta .
$$

where $(\xi, \eta) \in \mathbf{C}^{2}, \zeta \in \mathbf{C}^{n-2}$, and $\left|M_{1}(0)\right|>1$. Our convergence proof is inspired by the proof in Moser-Webster [MW83].

Proof. The last assertion follows from Lemma 4.7 and Lemma 4.9. Let us conjugate, simultaneously, each $F_{i}=\mathbf{D}_{i} x+f_{i}$ to $\hat{F}_{i}:=\hat{\mathbf{D}}_{i}(x) x$ by the action of $\Phi(x)=x+\phi(x)$ where $\phi(0)=0$ and $\phi^{\prime}(0)=0$. Here, $\hat{\mathbf{D}}_{i}(x)$ denotes the matrix $\operatorname{diag}\left(\hat{\mu}_{i 1}(x), \ldots, \hat{\mu}_{i n}(x)\right)$ and each $\hat{\mu}_{i j}(x)$ is a formal power series invariant under $D$, i.e. $\hat{\mu}_{i j}(x) \in \widehat{\mathcal{O}}_{n}^{D}$. We can assume that $\Phi$ does not have a non-zero component along the centralizer of $D$; indeed, by Lemma 4.9, we can assume that $\Phi$ is normalized w.r.t $D$. Then, for each $i=1, \ldots, \ell$, we have

$$
F_{i} \circ \Phi(x)=\mathbf{D}_{i} x+f_{i}(\Phi)(x)+\mathbf{D}_{i} \phi(x), \quad \Phi \circ \hat{F}_{i}(x)=\hat{\mathbf{D}}_{i}(x) x+\phi\left(\hat{F}_{i}\right)(x) .
$$

Equation $F_{i} \circ \Phi=\Phi \circ \hat{F}_{i}$ reads

$$
\left(\phi\left(\hat{\mathbf{D}}_{i}(x) x\right)-\mathbf{D}_{i} \phi(x)\right)+\left(\hat{\mathbf{D}}_{i}(x)-\mathbf{D}_{i}\right) x=f_{i}(\Phi)(x) \quad i=1, \ldots, \ell .
$$

Our convergence proof is based on two conditions: the existence of a formal $\phi \in \mathcal{C}^{\mathrm{c}}(D)$ that satisfies the above equation, and the Poincaré type condition on the linear part $D$. We already know that $\phi$ is unique. We shall project equation (4.4) along the "non-resonant" space (i.e. the space $\mathcal{C}^{\mathrm{c}}(D)$ of normalized mappings w.r.t. D). The mapping $\phi$ also solves this last equation and we shall majorize it using that projected equation.

Let us first decompose these equations along the "resonant" and "non-resonant" parts, i.e. $\mathcal{C}_{2}(D)$ and $\mathcal{C}_{2}^{\mathrm{c}}(D)$. Since $\phi=\sum_{Q \in \mathbf{N}^{n},|Q| \geq 2} \phi_{j, Q} x^{Q} e_{j}$ is normalized then $\phi_{j, Q}=0$ for some $Q \in \mathbf{N}^{n},|Q| \geq 2$ and $1 \leq j \leq n$, if we have $\mu_{m}^{Q}=\mu_{m j}$ for all $m$. We recall that, since each $\mathbf{D}_{i}$ is a diagonal matrix, then a map belongs to the centralizer of $D$ if and only if each monomial map of its Taylor expansion at the origin belongs to this centralizer as well. Since the $\hat{\mu}_{i j}$ is a formal invariant function then

$$
\phi\left(\hat{\mathbf{D}}_{i}(x) x\right)=\sum_{Q \in \mathbf{N}^{n},|Q| \geq 2} \phi_{j, Q} \hat{\mu}_{i}^{Q}(x) x^{Q} e_{j}=: \sum_{Q^{\prime} \in \mathbf{N}^{n},\left|Q^{\prime}\right| \geq 2} \psi_{j, Q^{\prime}} x^{Q^{\prime}} e_{j} .
$$

The latter contains only non-resonant terms, that is that if $\mu_{i}^{Q^{\prime}}=\mu_{i j}$ for all $i$, then $\psi_{j, Q^{\prime}}=0$. Indeed, $\hat{\mu}_{i}^{Q}(x)$ contains monomials of the form $x^{P}$ with $\mu_{i}^{P}=1$ for all $1 \leq i \leq \ell$. Hence, $\psi_{j, Q^{\prime}}$ is a linear combination of $\phi_{j, Q}$ such that $Q^{\prime}=Q+P$ with $\mu_{i}^{P}=1$ for all $i$. Therefore, if $\mu_{i}^{Q^{\prime}}=\mu_{i j}$ for all $i$, then for all these $Q^{\prime}$ 's, we have $\mu_{i}^{Q}=\mu_{i}^{Q^{\prime}}=\mu_{i j}$ for all $i$ so that $\phi_{j, Q}=0$; that is $\psi_{j, Q^{\prime}}=0$.

Hence, the projection on the resonant mappings in $\mathcal{C}_{2}(D)$ leads to

$$
\left(\hat{\mathbf{D}}_{i}(x)-\mathbf{D}_{i}\right) x=\left\{f_{i}(\Phi)(x)\right\}_{r e s}, \quad i=1, \ldots, \ell .
$$


Here for any formal mapping $g(x)=O\left(|x|^{2}\right)$ on $\mathbf{C}^{n}$, we define the projection on $\mathcal{C}_{2}(D)$ by

$$
(g(x))_{\text {res }}=\sum_{j} \sum_{\forall i, \mu_{i}^{Q}=\mu_{i j}} g_{j, Q} x^{Q} e_{j} .
$$

The projection $g$ onto $\mathcal{C}_{2}^{\mathrm{c}}(D)$ is defined as $g(x)-(g(x))_{\text {res }}$, i.e. it is the projection of $g$ on the non-resonant mappings.

Let us consider the projection on the non-resonant mappings. We first need to decompose power series according to a non-homogeneous equivalence relation on their coefficients. Let us define the equivalence relation on $\{1, \ldots, n\} \times \mathbf{N}^{n}$ by

$$
(j, Q) \sim(\tilde{\jmath}, \widetilde{Q}), \text { if } \mu_{i j}-\hat{\mu}_{i}^{Q}(x)=\mu_{i \tilde{\jmath}}-\hat{\mu}_{i}^{\widetilde{Q}}(x) \text { for all } 1 \leq i \leq \ell .
$$

Here the identities hold as formal power series. Let $\Delta$ be the set of the equivalence classes on the non-resonant multiindex set

$$
\left\{(j, Q) \in\{1, \ldots, n\} \times \mathbf{N}^{n}:\left(\mu_{1}^{Q}-\mu_{1 j}, \ldots, \mu_{\ell}^{Q}-\mu_{\ell j}\right) \neq 0,|Q|>1\right\} .
$$

If $\mu_{k}^{Q}-\mu_{k j} \neq 0$ for some $k$, clearly $\hat{\mu}_{k}^{Q}-\mu_{k j}$ is not identically zero. We can decompose any formal power series map $f$ along these equivalent classes and the resonant part of the mapping. Let $\delta \in \Delta$ and $f=\sum_{Q=\in \mathbf{N}^{n}, 1 \leq j \leq n} f_{j, Q} x^{Q} e_{j}$ with $f=O(2)$. We can write

$$
f_{\delta}(x):=\sum_{(j, Q) \in \delta} f_{j, Q} x^{Q} e_{j}, \quad \sum_{\delta \in \Delta} f_{\delta}(x) \prec \bar{f}(x) .
$$

We denote by $\widehat{\mathfrak{M}}_{n, \delta}^{n}$ the vector space of such maps. To a given equivalence class $\delta$, we associate a representative $\left(j_{\delta}, Q_{\delta}\right)$, and we shall identify an equation among $n$ equations in (4.4) for estimation. Since $\phi$ contains no resonant mappings, then

$$
\phi=\sum_{\delta \in \Delta} \phi_{\delta}
$$

Let us decompose the projection onto non-resonant mappings in $\mathcal{C}_{2}^{\mathrm{c}}(D)$ of equation (4.4) along each equivalence class $\delta$ as follows. Using the definition of the equivalence class $\Delta$, we obtain

$$
\left[\hat{\mu}_{i}^{Q_{\delta}}(x)-\mu_{i j_{\delta}}\right] \phi_{\delta}(x)=\left\{f_{i}(\Phi)\right\}_{\delta}(x), \quad \forall i=1, \ldots, \ell
$$

where $\{f\}_{\delta}$ denotes the projection of $f$ on $\widehat{\mathfrak{M}}_{n, \delta}^{n}$, defined by (4.7).

For each $\left(j_{\delta}, Q_{\delta}\right) \in \delta$, we know that $\mu_{k}^{Q_{\delta}}-\mu_{k j_{\delta}} \neq 0$ for some $k$. By the Poincaré type condition, there exist $i$ and $Q_{\delta}^{\prime} \in \mathbf{N}^{n}$ such that

$$
\mu_{i}^{Q_{\delta}^{\prime}}-\mu_{i j_{\delta}} \neq 0 ; \quad \mu_{m}^{Q_{\delta}^{\prime}}=\mu_{m}^{Q_{\delta}}, \quad \forall 1 \leq m \leq \ell ; \quad Q_{\delta}^{\prime}-Q_{\delta} \in \mathbf{N}^{n} \cup\left(-\mathbf{N}^{n}\right)
$$

and, furthermore, one of the following holds:

$$
\left|\mu_{i}^{Q_{\delta}^{\prime}}\right| \leq c d^{-\left|Q_{\delta}^{\prime}\right|}, \quad \text { or } \quad\left|\mu_{i}^{-Q_{\delta}^{\prime}}\right| \leq c d^{-\left|Q_{\delta}^{\prime}\right|} .
$$

Here, $d>1$ does not depend on $Q_{\delta}$. So, let us use the $i$ th equation of (4.9) to estimate $\phi_{\delta}$. We have, for that $i$,

$$
\phi_{\delta}=\left[\hat{\mu}_{i}^{Q_{\delta}}-\mu_{i j_{\delta}}\right]^{-1}\left\{f_{i}(\Phi)\right\}_{\delta} .
$$


Therefore, we have established the uniqueness of $\phi$ under (4.8) and (4.12), and under the condition that $\phi$ satisfies the equation when (4.4) is projected onto $\mathcal{C}^{\mathrm{c}}(D)$. The existence of $\phi$ is ensured by assumption. We now consider the convergence of $\phi$. By (4.10) we obtain $\hat{\mu}_{i}^{Q_{\delta}^{\prime}-Q_{\delta}}=1$. This allows us to rewrite (4.12) as

$$
\phi_{\delta}=\left[\hat{\mu}_{i}^{Q_{\delta}^{\prime}}-\mu_{i j_{\delta}}\right]^{-1}\left\{f_{i}(\Phi)\right\}_{\delta} .
$$

We majorize this power series.

Recall that $\hat{\mu}_{i j}(0)=\mu_{i j}$. Let us set $M_{i j}(x):=\mu_{i j}^{-1} \hat{\mu}_{i j}(x)$. We have $M_{i j}(0)=1$ and we decompose $M_{i j}(x)=\sum_{Q \in \mathbf{N}^{n}} M_{i j, Q} x^{Q}$. Let us set $\mu^{*}:=\max _{i j}\left\{\left|\mu_{i j}\right|,\left|\mu_{i j}^{-1}\right|\right\}$, and

$$
m_{i}=\sum_{Q \in \mathbf{N}^{n}} \max _{1 \leq j \leq n}\left|M_{i j, Q}\right| x^{Q}, \quad m=\sum_{Q \in \mathbf{N}^{n}} \max _{1 \leq i \leq \ell, 1 \leq j \leq n}\left|M_{i j, Q}\right| x^{Q} .
$$

Note that $m(0)=1$. Then $M_{i j} \prec m$ and

$$
M_{i j}^{-1}=\frac{1}{1+\left(M_{i j}-1\right)} \prec \frac{1}{1-(m-1)}=\frac{1}{2-m} .
$$

Here and in what follows, if $f(x)$ is a formal power series with $f(0)=0$, then for any number $a \neq 0, \frac{1}{a-f(x)}$ stands for the formal power series in $x$ for

$$
\frac{1}{a}\left\{1+\sum_{n=1}^{\infty}\left(a^{-1} f(x)\right)^{n}\right\}
$$

To simplify notation in (4.13), let us write $Q$ for $Q_{\delta}^{\prime}$ and $j$ for $j_{\delta}$. We want to show that,

$$
\left(\hat{\mu}_{i}^{Q}-\mu_{i j}\right)^{-1} \prec S(m-1) .
$$

Here $S(t)$ is a convergent power series independent of all $(j, Q)^{\prime} s$ of the form $\left(j_{\delta}, Q_{\delta}^{\prime}\right)^{\prime} s$. Fix $d_{1}$ with $1<d_{1}<d$. We consider the first case that $\mu^{*} c d^{-|Q|}>d_{1}^{-|Q|}$. Since $d>d_{1}$, we have only finitely many such $Q^{\prime} s$ (recall that each $Q$ has the form $Q_{\delta}^{\prime}$ ). The function $M_{i} \mapsto \mu_{i j}-\mu_{i}^{Q} M_{i}^{Q}$ is holomorphic in $M_{i} \in \mathbf{C}^{n}$ at $M_{i}=(1, \ldots, 1)$ and does not vanish at this point. Hence, the function

$$
\left(\mu_{i j}-\hat{\mu}_{i}^{Q}\right)^{-1}=\left(\mu_{i j}-\mu_{i}^{Q} M_{i}^{Q}\right)^{-1}
$$

is also holomorphic at $M_{i}=(1, \ldots, 1)$. For all $Q^{\prime} s$ in the first case, we have

$$
\left(\mu_{i j}-\hat{\mu}_{i}^{Q}\right)^{-1} \prec \frac{C}{1-C\left(\bar{M}_{i 1}-1+\cdots+\bar{M}_{i n}-1\right)} \prec \frac{C}{1-n C(m-1)} .
$$

Consider the second case that $\mu^{*} c d^{-|Q|} \leq d_{1}^{-|Q|}$. For the first case in (4.11), we obtain

$$
\begin{aligned}
\left(\hat{\mu}_{i}^{Q}-\mu_{i j}\right)^{-1} & =-\mu_{i j}^{-1}\left(1-\mu_{i j}^{-1} \mu_{i}^{Q} M_{i}^{Q}\right)^{-1} \prec \mu^{*}\left[1-\mu^{*} c d^{-|Q|} m^{|Q|}\right]^{-1} \\
& \prec \mu^{*}\left[1-d_{1}^{-|Q|} m^{|Q|}\right]^{-1} \prec \mu^{*}\left[1-d_{1}^{-1} m\right]^{-1} .
\end{aligned}
$$


For the second case in (4.11), we have

$$
\begin{aligned}
\left(\hat{\mu}_{i}^{Q}-\mu_{i j}\right)^{-1} & =-\mu_{i}^{-Q} M_{i}^{-Q}\left[1-\mu_{i j} \hat{\mu}_{i}^{-Q} M_{i}^{-Q}\right]^{-1} \\
& \prec c d^{-|Q|}(2-m)^{-|Q|}\left[1-\mu^{*} c d^{-|Q|}(2-m)^{-|Q|}\right]^{-1} \\
& \prec\left(\mu^{*}\right)^{-1} d_{1}^{-|Q|}(2-m)^{-|Q|}\left[1-d_{1}^{-|Q|}(2-m)^{-|Q|}\right]^{-1} \\
& \prec\left(\mu^{*}\right)^{-1}\left[1-d_{1}^{-1}(2-m)^{-1}\right]^{-1} .
\end{aligned}
$$

We have obtained the estimates for the second case. Therefore, we have shown that for any $Q=Q_{\delta}^{\prime}$ and $j=j_{\delta}$

$$
\left(\hat{\mu}_{i}^{Q}-\mu_{i j}\right)^{-1} \prec S(m-1) .
$$

Here $S(t)$ is a convergent power series independent of all $(j, Q)^{\prime} s$ of the form $\left(j_{\delta}, Q_{\delta}^{\prime}\right)^{\prime} s$.

Let us set

$$
f^{*}:=\sum_{Q \in \mathbf{N}^{n}} \max _{1 \leq i \leq \ell, 1 \leq j \leq n}\left|f_{i j, Q}\right| x^{Q} e_{j}
$$

By the definition of the equivalence relation on multiindices, we have

$$
\sum_{\delta \in \Delta} f_{\delta}^{*} \prec f^{*}
$$

According to (4.13) and (4.15), we have $\phi_{\delta} \prec S(m-1)\left\{f^{*}(\bar{\Phi})\right\}_{\delta}$. Now (4.7) and (4.16) imply

$$
\phi \prec S(m-1) f^{*}(\bar{\Phi}) .
$$

Let us project (4.5) onto the $k$ th components of $\mathcal{C}_{2}(D)$ as follows. For a power series map $g$, we define

$$
g_{r e s, k}(x)=\sum_{\mu^{Q}=\mu_{k}} g_{k, Q} x^{Q} .
$$

By the definition of $g_{\text {res }}$ in (4.6) $), g_{\text {res }}=\left(g_{r e s, 1}, \ldots, g_{r e s, n}\right)$. We have

$$
\mu_{i k}\left(M_{i, k}(x)-1\right) x_{k}=\left(\hat{\mu}_{i k}(x)-\mu_{i k}\right) x_{k}=\left\{f_{i k}(\Phi)\right\}_{r e s, k}(x) .
$$

Therefore, for all $1 \leq k \leq n$,

$$
(m-1) x_{k} \prec \frac{1}{\min _{i, j}\left|\mu_{i, j}\right|} f^{*}(\bar{\Phi}) .
$$

Let us set $\mu_{*}:=\frac{1}{\min _{i, j}\left|\mu_{i j}\right|}$. We set $x_{1}=t, \ldots, x_{n}=t$ in $\bar{\Phi}(x)$ and $m(x)$. Let $\phi(t), \bar{\Phi}(t)$, and $m(t)$ still denote $\phi(t, \ldots, t), \bar{\Phi}(t, \ldots, t)$, and $m(t, \ldots, t)$, respectively. Let

$$
t W(t):=\phi(t)+(m(t)-1) t .
$$

We have $W(0)=0, \phi(t) \prec t W(t)$, and $(m(t)-1) \prec W(t)$. From estimates (4.17) and (4.18), we obtain

$$
t W(t) \prec \mu_{*} f^{*}(\bar{\Phi}(t))+S(m(t)-1) f^{*}(\bar{\Phi}(t)) .
$$


Since $f_{i j}(x)=O\left(|x|^{2}\right)$, there exists a constant $c_{1}$ such that

$$
f^{*}(x) \prec \frac{c_{1}\left(\sum_{j} x_{j}\right)^{2}}{1-c_{1}\left(\sum_{j} x_{j}\right)} .
$$

Hence, estimate (4.19) reads

$$
\begin{aligned}
t W(t) & \prec\left(\mu_{*}+S(m(t)-1)\right) \frac{c_{1}(n(t+\phi))^{2}}{1-c_{1} n(t+\phi)} \\
& \prec\left(\mu_{*}+S(W(t))\right) \frac{c_{1} t^{2}(n(1+W(t)))^{2}}{1-c_{1} n t(1+W(t))} .
\end{aligned}
$$

Let us consider the equation in the unknown $U$ with $U(0)=0$ :

$$
U(t)\left(1-c_{1} n t(1+U(t))\right)=\left(\mu_{*}+S(W(t))\right) c_{1} t(n(1+U(t)))^{2} .
$$

According to the implicit function theorem, there exists a unique germ of holomorphic function $U(t)$, solution to (4.21) with $U(0)=0$. According to inequality (4.20), the function $W$ is dominated by $U: W(t) \prec U(t)$. This can be seen by induction on the degree of the Taylor polynomials at the origin. Therefore, $W$ converges at the origin. The theorem is proved.

\section{Real manifolds With an ABElian CR-Singularity}

Let us consider a real analytic manifold $M$ with a CR-singularity at the origin, which is an higher order perturbation of a product quadric. We assume that for its complexification $\mathcal{M}, \pi_{1}$ has $2^{p}$ deck transformations generated by $\left\{\tau_{11}, \ldots, \tau_{1 p}\right\}$. Let $\tau_{2 j}=\rho \circ \tau_{1 j} \circ \rho$.

Let us consider the following germs of holomorphic diffeomorphisms :

$$
\begin{gathered}
\sigma_{i}:=\tau_{1 i} \circ \tau_{2 i}, \quad 1 \leq i \leq e_{*}+h_{*}, \\
\sigma_{s}:=\tau_{1 s} \circ \tau_{2\left(s_{*}+s\right)}, \quad \sigma_{s+s_{*}}=\tau_{1\left(s+s_{*}\right)} \circ \tau_{2 s}, \quad e_{*}+h_{*}<s \leq p-s_{*} .
\end{gathered}
$$

Notice that the above property holds for quadrics of the complex case by Proposition 2.10. We assume that the linear parts $T_{i j}, S_{j}, S$ of $\tau_{i j}, \sigma_{j}, \sigma$ and $\rho$ are given by (2.32)-(2.35). The family $\left\{\sigma_{i}\right\}$ is reversible with respect to $\rho$. More precisely, we have the following relations

$$
\sigma_{i}^{-1}=\rho \sigma_{i} \rho, \quad 1 \leq i \leq e_{*}+h_{*} ; \quad \sigma_{s+s_{*}}^{-1}=\rho \sigma_{s} \rho, \quad e_{*}+h_{*}<s \leq p-s_{*} .
$$

Definition 5.1. We say that the manifold $M$ has an abelian CR-singularity at the origin if its complexification $\mathcal{M}$ has the maximum number of deck transformation and if the family $\left\{\sigma_{1}, \ldots, \sigma_{p}\right\}$ of germs of biholomorphisms at the origin of $\mathbf{C}^{2 p}$ is abelian, i.e.

$$
\sigma_{i} \sigma_{j}=\sigma_{j} \sigma_{i}
$$

The aim of this section is to show that such an analytic perturbation with an abelian CR-singularity and no hyperbolic component is holomorphically conjugate to a normal form. We shall give two proofs of this result. The first one rests on Moser-Webster result MW83][theorem 4.1] applied successively to each $\sigma_{i}$. It is to be emphasized that it is fortunate that we can apply such a result to our situation including the new type of CR singularity of complex type. The other one is based on the fact that the family $\left\{\sigma_{i}\right\}$ is formally completely integrable and their linear part is of Poincaré type. We then apply Theorem 4.13 . 
Before we apply the above theorem, let us first exhibit an example of real manifolds with an abelian $\mathrm{CR}$ singularity. We start with a Bishop surface $M_{0} \subset \mathrm{C}^{2}$ defined by $z_{2}=R\left(z_{1}, \overline{z_{1}}\right)$ with

$$
R\left(z_{1}, \overline{z_{1}}\right)=z_{1} \bar{z}_{1}+\gamma_{1}\left(z_{1}^{2}+\bar{z}_{1}^{2}\right)+O(3), \quad R\left(z_{1}, \bar{z}_{1}\right)=\bar{R}\left(\overline{z_{1}}, z_{1}\right) .
$$

Let $\tau_{1}^{0}, \tau_{2}^{0}$ be the Moser-Webster involutions of $M_{0}$. On the complexification $\mathcal{M}_{0}$ of $M_{0}$, the $z_{1}$ and $z_{2}:=R\left(z_{1}, w_{1}\right)$ are invariant by $\tau_{1}^{0}$. Condition (5.3) implies that $w_{2}:=\bar{R}\left(w_{1}, z_{1}\right)=z_{2}$ is also invariant by $\tau_{1}^{0}$. Analogously, $w_{1}, w_{2}$ and $z_{2}$ are invariant by $\tau_{2}^{0}$. We are ready to verify the following

Proposition 5.2. Let $R$ be given by (5.3) and let

$$
M: z_{3}=R\left(z_{1}, \overline{z_{1}}\right), \quad z_{4}=\left(z_{2}+2 \gamma_{2} \overline{z_{2}}+C\left(z_{2}, z_{3}, \bar{z}_{2}\right)\right)^{2},
$$

where $C\left(z_{2}, R\left(z_{1}, \overline{z_{1}}\right), \overline{z_{2}}\right)=O(3)$. Then $M$ has an abelian $C R$ singularity at 0 .

Proof. Let $\tau_{11}$ be defined by $\left(z_{1}^{\prime}, w_{1}^{\prime}\right)=\tau_{1}^{0}\left(z_{1}, w_{1}\right)$ and $\left(z_{2}^{\prime}, w_{2}^{\prime}\right)=\left(z_{2}, w_{2}\right)$. Let us verify that $\tau_{11}$ is a deck transformation of $\pi_{1}$, i.e. all $z_{j}$ are invariant by it. Obviously, $z_{2}, w_{2}$ are invariant by $\tau_{11}$. We know that $z_{1}, z_{3}=R\left(z_{1}, w_{1}\right)$ are invariant by $\tau_{1}^{0}$ and hence by $\tau_{11}$. On $\mathcal{M}, z_{4}=\left(z_{2}+2 \gamma_{2} w_{2}+C\left(z_{2}, z_{3}, w_{2}\right)\right)^{2}$ is then invariant by $\tau_{11}$. Therefore, $\tau_{11}$ is an involution and it fixes a hypersurface in $\mathcal{M}$. Let $\tau_{12}$ be defined by $\left(z_{1}^{\prime}, z_{2}^{\prime}, w_{1}^{\prime}\right)=\left(z_{1}, z_{2}, w_{1}\right)$ and

$$
z_{2}+2 \gamma_{2} w_{2}^{\prime}+C\left(z_{2}, R\left(z_{1}, w_{1}\right), w_{2}^{\prime}\right)=-z_{2}-2 \gamma_{2} w_{2}-C\left(z_{2}, R\left(z_{1}, w_{1}\right), w_{2}\right) .
$$

By the implicit function theorem, $w_{2}^{\prime}=-\gamma_{2}^{-1} z_{2}-w_{2}+f\left(z_{2}, R\left(z_{1}, w_{1}\right), w_{2}\right)$ with $f$ being convergent. It is clear that $\tau_{12}$ leaves $z_{j}$ invariant and it fixes a hypersurface.

For the abelian property, we note that $z_{2}, w_{2}$ and $R\left(z_{1}, w_{1}\right)$ are invariant by $\tau_{11}, \tau_{21}$. By a straightforward computation, we verity that $\tau_{11}$ and $\tau_{21}$ commute with $\tau_{12}$. Now $\tau_{21}$ and $\tau_{11}$ commute with $\tau_{22}=\rho \tau_{12} \rho$. This shows that $\tau_{11} \tau_{21}$ commutes with $\tau_{12} \tau_{22}$.

\subsection{Normal forms for abelian CR singularity.}

Theorem 5.3. Let $M$ be a germ of real analytic submanifold in $\mathbf{C}^{n}$ at an abelian CRsingularity at the origin. Suppose that $M$ is a higher order perturbation of a product quadric of which $\gamma_{1}, \ldots, \gamma_{p}$ satisfy (1.5). Suppose that $M$ does not have a hyperbolic component (i.e. $\left.e_{*} \geq 0, s_{*} \geq 0, h_{*}=0\right)$ and $\operatorname{Re} \gamma_{s}<1 / 2$. Then there exists a germ of biholomorphism $\psi$ that commutes with $\rho$ and such that, for $1 \leq i \leq p$ and $k=1,2$

$$
\psi^{-1} \circ \sigma_{i} \circ \psi:\left\{\begin{array}{l}
\xi_{i}^{\prime}=M_{i}(\xi \eta) \xi_{i} \\
\eta_{i}^{\prime}=M_{i}^{-1}(\xi \eta) \eta_{i} \\
\xi_{j}^{\prime}=\xi_{j} \\
\eta_{j}^{\prime}=\eta_{j}, \quad j \neq i,
\end{array} \quad \psi^{-1} \circ \tau_{k i} \circ \psi:\left\{\begin{array}{l}
\xi_{i}^{\prime}=\Lambda_{k i}(\xi \eta) \eta_{i} \\
\eta_{i}^{\prime}=\Lambda_{k i}^{-1}(\xi \eta) \xi_{i} \\
\xi_{j}^{\prime}=\xi_{j} \\
\eta_{j}^{\prime}=\eta_{j}, \quad j \neq i .
\end{array}\right.\right.
$$

Moreover, $\Lambda_{2 j}=\Lambda_{1 j}^{-1}$ and

$$
\begin{gathered}
\Lambda_{1 e}=\overline{\Lambda_{1 e} \circ \rho_{z}}, 1 \leq e \leq e_{*} ; \quad \Lambda_{1 s}^{-1}=\overline{\Lambda_{1\left(s+s_{*}\right)} \circ \rho_{z}}, e_{*}<s \leq p-s_{*}, \\
\rho_{z}: \zeta_{e} \rightarrow \bar{\zeta}_{e}, \quad \zeta_{s} \rightarrow \bar{\zeta}_{s+s_{*}}, \quad \zeta_{s+s_{*}} \rightarrow \bar{\zeta}_{s} .
\end{gathered}
$$


Proof. We will present two convergence proofs: one is based on a convergent theorem of Moser and Webster and another is based on Theorem 4.13. We first use some formal results obtained by Moser and Webster [MW83] and some results in section 4. The conditions of the theorem imply that, for all $i,\left|\mu_{i}\right| \neq 1$.

Since $M$ is a higher order perturbation of a product quadric, there are linear coordinates such that, for $1 \leq i \leq p$ and $k=1,2, \tau_{k i}$ and $\sigma_{i}$ are higher order perturbations of

$$
S_{i}:\left\{\begin{array}{l}
\xi_{i}^{\prime}=\mu_{i} \xi_{i} \\
\eta_{i}^{\prime}=\mu_{i}^{-1} \eta_{i} \\
\xi_{j}^{\prime}=\xi_{j} \\
\eta_{j}^{\prime}=\eta_{j}, \quad j \neq i,
\end{array} \quad T_{k i}:\left\{\begin{array}{l}
\xi_{i}^{\prime}=\lambda_{k i} \eta_{i} \\
\eta_{i}^{\prime}=\lambda_{k i}^{-1} \xi_{i} \\
\xi_{j}^{\prime}=\xi_{j} \\
\eta_{j}^{\prime}=\eta_{j}, \quad j \neq i .
\end{array}\right.\right.
$$

For elliptic coordinates, this was computed in [MW83] and recalled in (2.21). For complex coordinates, this is computed in (2.27) and (2.29). Recall that $\sigma_{1}, \ldots, \sigma_{p}$ are defined by (5.1)-(5.2) Since $\left|\mu_{1}\right| \neq 1$, then by theorem 4.1 of Moser-Webster ([MW83]), there is a unique convergent transformation $\psi_{1}$ normalized w.r.t. $S_{1}$ (see Definition 4.5) such that $\sigma_{1}^{*}:=\psi_{1}^{-1} \circ \sigma_{1} \circ \psi_{1}$ and $\tau_{i 1}^{*}:=\psi_{1}^{-1} \circ \tau_{i 1} \circ \psi_{1}$ are given by

$$
\sigma_{1}^{*}:\left\{\begin{array}{l}
x_{1}^{\prime}=M_{1}(\xi, \eta) \xi_{1} \\
\eta_{1}^{\prime}=M_{1}^{-1}(\xi, \eta) \eta_{1} \\
\xi_{j}^{\prime}=\xi_{j} \\
\eta_{j}^{\prime}=\eta_{j}, \quad j \neq 1,
\end{array} \quad \tau_{k 1}^{*}:\left\{\begin{array}{l}
\xi_{1}^{\prime}=\Lambda_{k 1}(\xi, \eta) \eta_{1} \\
\eta_{1}^{\prime}=\Lambda_{k 1}^{-1}(\xi, \eta) \xi_{1} \\
\xi_{j}^{\prime}=\xi_{j} \\
\eta_{j}^{\prime}=\eta_{j}, \quad j \neq 1 .
\end{array}\right.\right.
$$

Here $k=1,2$. It is a simple fact (e.g. see Lemma 4.9, $D=\left\{S_{1}\right\}$ ) that there is a unique $\phi_{1} \in \mathcal{C}^{c}\left(S_{1}\right)$ such that $\phi_{1}^{-1} \sigma_{1} \phi_{1}$ is in the centralizer of $S_{1}$. Therefore, $\phi_{1}=\psi_{1}$ is also convergent.

Furthermore, we have $M_{1}(\xi, \eta)=\Lambda_{11}(\xi, \eta) \Lambda_{21}^{-1}(\xi, \eta)$; and $\Lambda_{11}, \Lambda_{21}, M_{1}$ are invariant by $S_{1}$. In the new coordinates, let us denote $\tau_{i m}, \sigma_{m}$ by the same symbols for $m>1$. However, $\sigma_{1}=\sigma_{1}^{*}$ and $\tau_{k 1}=\tau_{k 1}^{*}$. Since each $\sigma_{m}$ commutes with $\sigma_{1}$, then $\sigma_{m}$ is in the centralizer of $S_{1}$. Indeed, according to [MW83][Lemma 3.1](or Lemma 4.7 with $D=\left\{S_{1}\right\}$ ), we can decompose $\sigma_{m}=\sigma_{m}^{1} \sigma_{m}^{0}$ where $\sigma_{m}^{1}$ is normalized w.r.t $S_{1}$ and $\sigma_{m}^{0}$ is in the centralizer of $S_{1}$. Write $\sigma_{1} \sigma_{m}=\sigma_{m} \sigma_{1}$ as

$$
\left(\sigma_{m}^{1}\right)^{-1} \sigma_{1} \sigma_{m}^{1}=\sigma_{m}^{0} \sigma_{1}\left(\sigma_{m}^{0}\right)^{-1}
$$

Since $\sigma_{m}^{0} \sigma_{1}\left(\sigma_{m}^{0}\right)^{-1}$ belongs to $\mathcal{C}\left(S_{1}\right)$, so does $\left(\sigma_{m}^{1}\right)^{-1} \sigma_{1} \sigma_{m}^{1}$. Then applying the uniqueness of $\psi_{1}$ stated earlier to $\sigma_{m}^{1}$, we conclude that $\sigma_{m}^{1}=I$ and $\sigma_{m}=\sigma_{m}^{0}$ is in the centralizer of $S_{1}$.

Let us verify that $\sigma_{m}^{0}$ or in general each (formal) transformation $\varphi$ in $\mathcal{C}\left(S_{1}\right)$ preserves the form of $\sigma_{1}^{*}$ and $\tau_{i 1}^{*}$. Indeed, $\varphi^{-1}$ commutes with $S_{1}$ too. Thus $\varphi^{-1} \sigma_{1}^{*} \varphi$ commutes with $S_{1}$ and its linear part is $S_{1}$. The linear part of $\varphi$ must preserve the eigenspaces of $S_{1}$ and hence it is given by

$$
\xi_{1} \rightarrow a \xi_{1}, \quad \eta_{1} \rightarrow b \eta_{1}, \quad\left(\xi_{*}, \eta_{*}\right) \rightarrow \phi\left(\xi_{*}, \eta_{*}\right)
$$

for $\xi_{*}=\left(\xi_{2}, \ldots, \xi_{n}\right)$ and $\eta_{*}=\left(\eta_{2}, \ldots, \eta_{n}\right)$. The linear part of $\tau_{k 1}^{*}$ is given by

$$
\xi_{1} \rightarrow \lambda_{k 1} \eta_{1}, \quad \eta_{1} \rightarrow \lambda_{k 1}^{-1} \xi_{1}, \quad\left(\xi_{*}, \eta_{*}\right) \rightarrow\left(\xi_{*}, \eta_{*}\right) .
$$

By a simple computation, the linear part of $\varphi^{-1} \tau_{k 1}^{*} \varphi$ still has this form with $\lambda_{k 1} \frac{b}{a}$ instead of $\lambda_{k 1}$. According to [MW83][lemma 3.2], there a unique normalized mapping $\Psi$ that 
normalizes $\varphi^{-1} \sigma_{1}^{*} \varphi$ and the $\varphi^{-1} \tau_{k 1}^{*} \varphi^{\prime}$ 's. According to the uniqueness property of Lemma 4.8, $\Psi=I d$. Therefore, $\varphi$ preserves the forms of $\tau_{i 1}^{*}$ and $\sigma_{1}^{*}$.

Let $\psi_{2}$ be the unique biholomorphic map normalized w.r.t. $S_{2}$ such that $\psi_{2}^{-1} \sigma_{2} \psi_{2}=\sigma_{2}^{*}$ and $\psi_{2}^{-1} \tau_{k 2} \psi_{2}=\tau_{k 2}^{*}$ are in the normal form :

$$
\sigma_{2}^{*}:\left\{\begin{array}{l}
\xi_{2}^{\prime}=M_{2}(\xi, \eta) \xi_{2} \\
\eta_{2}^{\prime}=M_{2}^{-1}(\xi, \eta) \eta_{2} \\
\xi_{j}^{\prime}=\xi_{j} \\
\eta_{j}^{\prime}=\eta_{j}, \quad j \neq 2,
\end{array} \quad \tau_{k 2}^{*}:\left\{\begin{array}{l}
\xi_{2}^{\prime}=\Lambda_{k 2}(\xi, \eta) \eta_{2} \\
\eta_{2}^{\prime}=\Lambda_{k 2}^{-1}(\xi, \eta) \xi_{2} \\
\xi_{j}^{\prime}=\xi_{j} \\
\eta_{j}^{\prime}=\eta_{j}, \quad j \neq 2 .
\end{array}\right.\right.
$$

Here $k=1,2$, and $M_{2}$ and $\Lambda_{k 2}$ are invariant by $S_{2}$. Since $\sigma_{2}$ commutes with $S_{1}$, we have

$$
\left(S_{1}^{-1} \psi_{2} S_{1}\right)^{-1} \circ \sigma_{2} \circ\left(S_{1}^{-1} \psi_{2} S_{1}\right)=S_{1}^{-1} \sigma_{2}^{*} S_{1} \text {. }
$$

Note that $S_{1}^{-1} \sigma_{2}^{*} S_{1}$ (resp. $S_{1}^{-1} \tau_{k 2}^{*} S_{1}$ ) has the form (5.9) in which $M_{2}$ (resp. $\Lambda_{k 2}$ ) is replaced by $M_{2} \circ S_{1}$ (resp. $\Lambda_{k 2} \circ S_{1}$ ). In other words $S_{1}^{-1} \sigma_{2}^{*} S_{1}$ and $S_{1}^{-1} \tau_{k 2}^{*} S_{1}$ are still of the form (5.9). Since $S_{1}$ is diagonal, then $S_{1}^{-1} \psi_{2} S_{1}$ remains normalized w.r.t. $S_{2}$. Applying the above uniqueness on $\psi_{2}$ for $\sigma_{2}$, we conclude that $\psi_{2}=S_{1}^{-1} \psi_{2} S_{1}$. This shows that $\psi_{2}$ preserves the forms of $\tau_{k 1}^{*}$ and $\sigma_{1}^{*}$. By the same argument as above, we have $\sigma_{m}^{*} \in \mathcal{C}\left(S_{1}, S_{2}\right)$.

In summary, we have found holomorphic coordinates so that $\tau_{i j}=\tau_{i m}^{*}$ and $\sigma_{m}=\sigma_{m}^{*}$ for $m=1,2$. As mentioned previously, we know that $\sigma_{1}^{*}, \sigma_{2}^{*}, \sigma_{3}, \ldots, \sigma_{m}$ commute with $S_{1}$ and $S_{2}$. In particular, $M_{1}, M_{2}$ are invariant by $S_{1}, S_{2}$. Repeating this procedure, we find a holomorphic map $\phi$ so that all $\phi^{-1} \sigma_{j} \phi=\sigma_{j}^{*}$ and $\phi^{-1} \tau_{k j} \phi=\tau_{k j}^{*}$ are in the normal forms. Furthermore, $M_{i}$ and $\Lambda_{k, i}$ are invariant by $\mathcal{S}=\left\{S_{1}, \ldots, S_{p}\right\}$.

By Lemma 4.7, we decompose $\phi=\phi_{1} \phi_{0}^{-1}$ where $\phi_{1}$ is normalized w.r.t. $\mathcal{S}$ and $\phi_{0}$ is in the centralizer of $\mathcal{S}$. Then $\phi_{1}^{-1} \sigma_{j} \phi_{1}=\sigma_{j}^{*}$ and $\phi_{1}^{-1} \tau_{i j} \phi_{1}=\tau_{i j}^{*}$ are in the normal forms, since $\phi_{0}$ commutes with $S_{j}$. We want to show that $\phi_{1}$ commutes with $\rho$.

Note that $\sigma_{e}^{-1}=\rho \sigma_{e} \rho$ and $\sigma_{s+s_{*}}^{-1}=\rho \sigma_{s} \rho$. Thus $\left(\rho \phi_{1} \rho\right)^{-1} \sigma_{j}\left(\rho \phi_{1} \rho\right)=\tilde{\sigma}_{j}^{*}$ where $\tilde{\sigma}_{e}^{*}:=$ $\rho\left(\sigma_{e}^{*}\right)^{-1} \rho$ and $\tilde{\sigma}_{s}^{*}:=\rho\left(\sigma_{s+s *}^{*}\right)^{-1} \rho$. It is easy to see that $\rho \phi_{1} \rho$ is still normalized w.r.t. $\mathcal{S}$ (see also Definition 6.4). By Lemma 4.8, we know that there is a unique normalized formal mapping $\phi_{1}$ such that $\phi_{1}^{-1} \sigma_{j} \phi_{1}$ are in the centralizer of $\mathcal{S}$. Since $\tilde{\sigma}_{j}^{*}$ belongs to the centralizer of $\mathcal{S}$, then we have $\rho \phi_{1} \rho=\phi_{1}$.

Now, $\tau_{2 j}^{*}=\rho \tau_{1 j}^{*} \rho$ follows from $\tau_{2 j}=\rho \tau_{1 j} \rho$. This shows that

$$
\Lambda_{2 e}=\overline{\Lambda_{1 e}^{-1} \circ \rho_{z}}, \quad \Lambda_{2 s}=\overline{\Lambda_{1\left(s+s_{*}\right)} \circ \rho_{z}}, \quad \Lambda_{2\left(s+s_{*}\right)}=\overline{\Lambda_{1 s} \circ \rho_{z}},
$$

where $1 \leq e \leq e_{*}$ and $e_{*}<s \leq p-s_{*}$. Let $\phi_{2}$ be defined by

$$
\xi_{j}^{\prime}=\left(\Lambda_{1 j}^{1 / 2} M_{j}^{1 / 4}\right)(\xi \eta) \xi_{j}, \quad \eta_{j}^{\prime}=\left(\Lambda_{1 j}^{-1 / 2} M_{j}^{-1 / 4}\right)(\xi \eta) \eta_{j}, \quad 1 \leq j \leq p .
$$

For a suitable choice of the roots, we have $\phi_{2} \rho=\rho \phi_{2}$. Furthermore, $\phi_{2}$ preserves all invariant functions of $\mathcal{S}$. Hence, each $\phi_{2}^{-1} \circ \phi_{1}^{-1} \circ \tau_{k i} \circ \phi_{1} \circ \phi_{2}$ has the form $\tau_{k j}^{*}$ stated in Theorem 5.3.

We now present another proof by using the more general Theorem 4.13.

Note that the above proof is valid at the formal level without using the convergence result of Moser and Webster. More specifically, if $\tau_{i j}$ are given by formal power series with $\sigma_{1}, \ldots, \sigma_{p}$ commuting pairwise, there exists a formal map $\psi$ that is tangent to the identity 
and commutes with $\rho$ such that (5.5) holds. Since each $\mu_{j}$ is not a root of unity, then (5.5) implies that the conjugate family $\left\{\sigma_{m}^{*}\right\}$ is a completely integrable normal form.

Let $\sigma_{i}$ be defined as above. Let $S_{i}$ be its linear part at the origin of $\mathbf{C}^{n}$. The eigenvalues $\left\{\mu_{i j}\right\}_{1 \leq j \leq n}$ of $S_{i}$ are either $\mu_{i}, \mu_{i}^{-1}$ or 1 . More precisely, if $Q \in \mathbf{N}^{n},|Q| \geq 2$ then

$$
\mu_{m}^{Q}-\mu_{m j}=\mu_{m}^{q_{m}-q_{m+p}}-\left\{\begin{array}{l}
\mu_{m} \text { if } j=m \\
\mu_{m}^{-1} \text { if } j=m+p \\
1 \quad \text { otherwise }
\end{array}\right.
$$

We need to verify the condition that the family of linear part $\left\{S_{1}, \ldots, S_{p}\right\}$ is of the Poincaré type. So we can apply Theorem 4.13 .

Suppose that $(j, Q) \in\{1, \ldots, 2 p\} \times \mathbf{N}^{2 p}$ satisfies $\mu_{l}^{Q}-\mu_{l j} \neq 0$ for some $1 \leq l \leq 2 p$. Set $d=\left\{\min _{i} \max \left(\left|\mu_{i}\right|,\left|\mu_{i}^{-1}\right|\right)\right\}^{1 /(2 p)}$. We define

$$
Q^{\prime}=Q-\sum_{i=1}^{p} \min \left(q_{i}, q_{i+p}\right)\left(e_{i}+e_{i+p}\right):=\left(q_{1}^{\prime}, \ldots, q_{2 p}^{\prime}\right) .
$$

Then $\mu_{i}^{Q}=\mu_{i}^{Q^{\prime}}$ for all $i$. Take $i=l$ if $\left|Q^{\prime}\right| \leq 2 p$. In this case, we easily get

$$
\mu_{i}^{Q^{\prime}}-\mu_{i j} \neq 0, \quad\left|\mu_{i}^{Q^{\prime}}\right|>c^{-1} d^{\left|Q^{\prime}\right|}
$$

by choosing a sufficiently large $c$. Assume that $\left|Q^{\prime}\right|>2 p$. Take $i$ such that

$$
q_{i}^{\prime}+q_{i+p}^{\prime}=\max _{k}\left(q_{k}^{\prime}+q_{k+p}^{\prime}\right) .
$$

Then $q_{i}^{\prime}+q_{i+p}^{\prime} \geq\left|Q^{\prime}\right| / p>2$. By (5.10), we get the first equality in (5.11). We note that $\left(q_{i}^{\prime}, q_{i+p}^{\prime}\right)=\left(q_{i}, 0\right)$ or $\left(0, q_{i+p}\right)$. Thus

$$
\max \left(\left|\mu_{i}^{Q^{\prime}}\right|,\left|\mu_{i}^{-Q^{\prime}}\right|\right)=\left(\max \left(\left|\mu_{i}\right|,\left|\mu_{i}\right|^{-1}\right)\right)^{q_{i}^{\prime}+q_{i+p}^{\prime}} \geq d^{\left|Q^{\prime}\right|} .
$$

This shows that $\left\{D \sigma_{1}(0), \ldots, D \sigma_{p}(0)\right\}$ is of the Poincaré type.

We now apply Theorem 4.13 as follows. We decompose $\psi=\psi_{1} \psi_{0}^{-1}$ such that $\psi_{1} \in$ $\mathcal{C}^{\mathrm{c}}\left(S_{1}, \ldots, S_{p}\right)$ and $\psi_{0} \in \mathcal{C}\left(S_{1}, \ldots, S_{p}\right)$. Then each $\sigma_{i}^{*}=\psi_{1}^{-1} \sigma_{i} \psi_{1}$ still has the form in (5.5); in particular, $\left\{\sigma_{1}^{*}, \ldots, \sigma_{p}^{*}\right\}$ is a completely integrable formal normal form. By Theorem4.13, $\psi_{1}$ is convergent. Now, $\psi_{1}^{-1} \tau_{k j} \psi_{1}=\psi_{0}^{-1}\left(\psi^{-1} \tau_{k j} \psi\right) \psi_{0}$ are still of the form (5.5); however (5.6) and $\Lambda_{2 j} \Lambda_{1 j}=1$ might not hold. As in the first proof, we can verify that $\psi_{1} \rho=\rho \psi_{1}$. Applying another change of coordinates that commutes with $\rho$ and each $S_{j}$ as before, we achieve (5.5) -(5.6) and $\Lambda_{2 j} \Lambda_{1 j}=1$. The proof of the theorem is complete.

As a corollary of Theorem [5.3, we have the following normal form for real submanifolds. In order to study the holomorphic flatness and hull of holomorphy, we choose a realization similar to the case of Moser-Webster for $p=1$.

Theorem 5.4. Let $M$ be as in Theorem 5.3. Then $M$ is holomorphically equivalent to

$$
\widehat{M}: z_{p+j}=\Lambda_{1 j}(\zeta) \zeta_{j}, \quad 1 \leq j \leq p
$$


where $\zeta=\left(\zeta_{1}, \ldots, \zeta_{p}\right)$ are the convergent solutions to

$$
\begin{aligned}
\zeta_{e} & =A_{e}(\zeta)\left|z_{e}\right|^{2}-B_{e}(\zeta)\left(z_{e}^{2}+\bar{z}_{e}^{2}\right) \\
\zeta_{s} & =A_{s}(\zeta) z_{s} \bar{z}_{s+s_{*}}-B_{s}(\zeta)\left(z_{s}^{2}+\Lambda_{1 s}^{2}(\zeta) \bar{z}_{s+s_{*}}^{2}\right), \\
\zeta_{s+s_{*}} & =A_{s+s_{*}} \bar{z}_{s} z_{s+s_{*}}-B_{s+s_{*}}(\zeta)\left(z_{s+s_{*}}^{2}+\Lambda_{1\left(s+s_{*}\right)}^{2}(\zeta) \bar{z}_{s}^{2}\right) .
\end{aligned}
$$

Here $\Lambda_{1 j}(\zeta)=\lambda_{j}+O(\zeta)(1 \leq j \leq p)$ satisfy (5.6) and

$$
\begin{gathered}
A_{e}=\frac{1+\Lambda_{1 e}^{2}}{\left(1-\Lambda_{1 e}^{2}\right)^{2}}, \quad A_{j}=\frac{\Lambda_{1 j}+\Lambda_{1 j}^{3}}{\left(1-\Lambda_{1 j}^{2}\right)^{2}}, j=s, s+s_{*}, \\
B_{j}=\frac{\Lambda_{1 j}}{\left(1-\Lambda_{1 j}^{2}\right)^{2}}, \quad j=e, s, s+s_{*} .
\end{gathered}
$$

In particular, $\widehat{M}$ is contained in $z_{p+e}=\bar{z}_{p+e}$ and $z_{p+s} \tilde{\Lambda}_{1 s}^{2}\left(z^{\prime \prime}\right)=\bar{z}_{p+s+s_{*}}$, where $\zeta_{j}=$ $z_{p+j} \tilde{\Lambda}_{1 j}\left(z^{\prime \prime}\right), 1 \leq j \leq p$, is the inverse mapping of $z_{p+j}=\zeta_{j} \Lambda_{1 j}(\zeta), 1 \leq j \leq p$.

Proof. We use a realization which is different from (2.17). We assume that $M$ already has the normal form as in Theorem 5.3 . Thus for $j=1, \ldots, p$, we have

$$
\tau_{1 j}: \xi_{j}^{\prime}=\Lambda_{1 j}(\xi \eta) \eta_{j}, \quad \eta_{j}^{\prime}=\Lambda_{1 j}^{-1}(\xi \eta) \xi_{j}, \quad\left(\xi_{k}^{\prime}, \eta_{k}^{\prime}\right)=\left(\xi_{k}, \eta_{k}\right), \quad k \neq j .
$$

Let us define

$$
f_{j}(\xi, \eta)=\xi_{j}+\xi_{j} \circ \tau_{1 j}, \quad g_{j}=\overline{f_{j} \circ \rho}, \quad 1 \leq j \leq p .
$$

The latter implies that the biholomorphic mapping $\varphi(\xi, \eta)=(f(\xi, \eta), g(\xi, \eta))$ transforms $\rho$ into the standard complex conjugation $\left(z^{\prime}, w^{\prime}\right) \rightarrow\left(\bar{w}^{\prime}, \bar{z}^{\prime}\right)$. Define

$$
F_{j}(\xi, \eta)=\xi_{j} \circ \tau_{1 j}(\xi, \eta) \xi_{j}, \quad 1 \leq j \leq p .
$$

Using the expressions of $\tau_{1 j}$ given by (5.18), we verify that $f_{j}$ and $F_{j}$ are invariant by $\tau_{1 k}$. Note that the linear part of $f_{j}(\xi, \eta)$ is $\xi_{j}+\lambda_{j} \eta_{j}$ for $1 \leq j \leq p$, and the quadratic part of $F_{j}(\xi, \eta)$ is $\lambda_{j} \xi_{j}^{2}$. By Lemma 2.5, $f_{1}, \ldots, f_{p}$ and $F_{1}, \ldots, F_{p}$ generate all invariant functions of $\left\{\tau_{11}, \ldots, \tau_{1 p}\right\}$.

Using $\overline{\Lambda_{1 e} \circ \rho_{z}}=\Lambda_{1 e}$ and $\overline{\Lambda_{1 s} \circ \rho_{z}}=\Lambda_{1\left(s+s_{*}\right)}^{-1}$, rewrite $z_{j}=f_{j}(\xi, \eta), w_{j}=g_{j}(\xi, \eta)$ as

$$
\begin{aligned}
\xi_{e} & =\frac{z_{e}-\Lambda_{1 e}(\xi \eta) w_{e}}{1-\Lambda_{1 e}^{2}}, & \eta_{e} & =\frac{w_{e}-\Lambda_{1 e}(\xi \eta) z_{e}}{1-\Lambda_{1 e}^{2}}, \\
\xi_{s} & =\frac{z_{s}-\Lambda_{1 s}^{2}(\xi \eta) w_{s+s_{*}}}{1-\Lambda_{1 s}^{2}(\xi \eta)}, & \eta_{s} & =\frac{\Lambda_{1 s}(\xi \eta)\left(w_{s+s_{*}}-z_{s}\right)}{1-\Lambda_{1 s}^{2}(\xi \eta)}, \\
\xi_{s+s_{*}} & =\frac{z_{s+s_{*}}-\Lambda_{1\left(s+s_{*}\right)}^{2}(\xi \eta) w_{s}}{1-\Lambda_{1\left(s+s_{*}\right)}^{2}(\xi \eta)}, & \eta_{s+s_{*}} & =\frac{\Lambda_{1\left(s+s_{*}\right)}(\xi \eta)\left(w_{s}-z_{s+s_{*}}\right)}{1-\Lambda_{1\left(s+s_{*}\right)}^{2}(\xi \eta)} .
\end{aligned}
$$

Using the above formulas and $w_{j}=\bar{z}_{j}$, we compute $\zeta_{j}=\xi_{j} \eta_{j}$ to obtain (15.13)-(5.15).

Note that $F_{j}(\xi, \eta)=\zeta_{j} \Lambda_{1 j}(\zeta)$. This shows that $z_{p+j}=F_{j} \circ \varphi^{-1}\left(z^{\prime}, \bar{z}^{\prime}\right)$ have the form (5.12). Again, we use the formula of $\tau_{1 k}$ to verify that $z=\left(z^{\prime}, z^{\prime \prime}\right)$ are invariant by all $\varphi \tau_{1 k} \varphi^{-1}$. On the other hand, $z=\left(z^{\prime}, z^{\prime \prime}\right)$ generate invariant functions of the deck transformations of $\pi_{1}$ for the complexification of $\hat{M}$ given by (5.12). This shows that $\left\{\varphi \tau_{11} \varphi^{-1}, \ldots, \varphi \tau_{1 p} \varphi^{-1}\right\}$ and the deck transformations of $\pi_{1}$, of which each family consists of commuting involutions, 
have the same invariant functions. By Lemma 2.5, we know that the two families must be identical. This shows that (5.12) is a realization for $\left\{\tau_{11}, \ldots, \tau_{1 p}, \rho\right\}$.

To verify the last assertion of the theorem, we first note that by (5.6) the solutions $\zeta_{1}, \ldots, \zeta_{p}$ to (5.13) -(5.15) satisfy $\zeta_{e}=\bar{\zeta}_{e}$ and $\zeta_{s+s_{*}}=\bar{\zeta}_{s}$. By (5.6), on $M$ we have $\bar{z}_{p+e}=$ $\overline{\Lambda_{1 e}(\zeta) \bar{\zeta}_{e}}=z_{p+e}$. Also $\bar{z}_{p+s+s_{*}}={\overline{\Lambda_{1\left(s+s_{*}\right)}(\zeta)}}_{\zeta_{s+s_{*}}}=\Lambda_{1 s}^{-1}(\zeta) \zeta_{s}=\Lambda_{1 s}^{-2}(\zeta) z_{p+s}$. From (15.12), solve $\zeta_{j}=\tilde{\Lambda}_{1 j}\left(z^{\prime \prime}\right) z_{p+j}(1 \leq j \leq p)$ to obtain $\Lambda_{1 j}^{-1}(\zeta)=\tilde{\Lambda}_{1 j}\left(z^{\prime \prime}\right)$. The proof is complete.

5.2. Hull of holomorphy for the abelian CR singularity. Let $X$ be a subset of $\mathbf{C}^{n}$. We define the hull of holomorphy of $X$, denoted by $\mathcal{H}(X)$, to be the intersection of domains of holomorphy in $\mathbf{C}^{n}$ that contain $X$. Let $B_{r}^{n}$ be the ball in $\mathbf{C}^{n}$ of radius $r$ and centered at the origin.

By Theorem 5.4, we assume that $M$ has pure elliptic type and it is equivalent to

$$
M: z_{p+j}=\Lambda_{1 j}(\zeta) \zeta_{j}, \quad 1 \leq j \leq p
$$

where $\zeta_{j}=\zeta_{j}\left(z^{\prime}\right)(j=1, \ldots, p)$ are the convergent real-valued solutions to (5.13). For $\zeta \in \mathbf{R}^{p}$ with small $|\zeta|$, we know that $\Lambda_{1 j}(\zeta)>1$.

Near the origin in $\mathbf{R}^{p}$, we define a real analytic diffeomorphism:

$$
R: \zeta \rightarrow\left(\Lambda_{11}(\zeta) \zeta_{1}, \ldots, \Lambda_{1 p}(\zeta) \zeta_{p}\right)
$$

If $\epsilon$ is small enough, for each $x^{\prime \prime} \in[0, \epsilon]^{p}$, we can define $\zeta=R^{-1}\left(x^{\prime \prime}\right)$. Note that $R$ sends $\zeta_{j}=0$ into $x_{p+j}=0$ for each $j$. We can write

$$
R^{-1}\left(x^{\prime \prime}\right)=\left(x_{p+1} S_{1}\left(x^{\prime \prime}\right), \ldots, x_{2 p} S_{p}\left(x^{\prime \prime}\right)\right)
$$

with $S_{j}(0)>0$. Then $M \cap\left\{z^{\prime \prime}=x^{\prime \prime}\right\}$ is given by (15.12)-(5.15). For $x^{\prime \prime} \in[0, \epsilon]^{p}$ let $D_{j}\left(x^{\prime \prime}\right)$ be the compact set in the complex plane whose boundary is defined by the $j$ th equation in (5.12)-(5.15) where $\zeta=R^{-1}\left(x^{\prime \prime}\right)$. When $x_{p+j}>0$, the boundary of $D_{j}\left(x^{\prime \prime}\right)$ is an ellipse with

$$
D_{j}\left(x^{\prime \prime}\right) \subset B_{C_{1} \sqrt{x_{p+j}}}^{1} .
$$

Here and in what follows constants will depend only on $\lambda_{1}, \ldots, \lambda_{p}$. Thus

$$
D\left(x^{\prime \prime}\right):=D_{1}\left(x^{\prime \prime}\right) \times \cdots \times D_{p}\left(x^{\prime \prime}\right) \times\left\{x^{\prime \prime}\right\} \subset \mathbf{C}^{p} \times \mathbf{R}^{p}
$$

is a product of ellipses and its dimension equals the number of positive numbers among $x_{p+1}, \ldots, x_{2 p}$. We will call $D\left(x^{\prime \prime}\right)$ an analytic polydisc and

$$
\partial^{*} D\left(x^{\prime \prime}\right):=\partial D_{1}\left(x^{\prime \prime}\right) \times \cdots \times \partial D_{p}\left(x^{\prime \prime}\right) \times\left\{x^{\prime \prime}\right\}
$$

its distinguished boundary which is contained in $M$. Set $D\left(0^{\prime \prime}\right)=\partial^{*} D\left(0^{\prime \prime}\right)=\{0\}$. Thus, $M$ is foliated by $\partial^{*} D\left(x^{\prime \prime}\right)$ as $x^{\prime \prime}$ vary in $[0, \epsilon]^{p}$ and $\epsilon$ is sufficiently small. Specifying the $\epsilon$ later, we will use this foliation and Hartogs' figures in analytic polydiscs to find the local hull of holomorphy of $M$ at the origin.

As $x^{\prime \prime}$ vary in $[0, \epsilon]^{p}$, let $M_{\epsilon}$ be the union of $\partial^{*} D\left(x^{\prime \prime}\right)$, and $\mathcal{H}_{\epsilon}$ the union of $D\left(x^{\prime \prime}\right)$. Both $\mathcal{H}_{\epsilon}$ and $M_{\epsilon}$ are compact subsets in $\mathbf{C}^{2 p}$. Note that

$$
B_{\epsilon_{*}}^{2 p}+M_{\epsilon}:=\left\{a+b: a \in B_{\epsilon_{*}}^{2 p}, b \in M_{\epsilon}\right\}
$$


is contained in a given neighborhood of $M_{\epsilon}$, if $\epsilon_{*}$ is sufficiently small. Analogously, $B_{\epsilon_{*}}^{2 p}+\mathcal{H}_{\epsilon}$ is a connected open neighborhood of $\mathcal{H}_{\epsilon}$. Let us first verify that a function that is holomorphic in a connected neighborhood of $M_{\epsilon}$ in $\mathbf{C}^{2 p}$ extends holomorphically to a neighborhood of $\mathcal{H}_{\epsilon}$ such that the extension agrees with the original function on a possibly smaller neighborhood of $M_{\epsilon}$. Assume that $f$ is holomorphic in a neighborhood $\mathcal{U}$ of $\partial_{\epsilon}^{*} D:=\cup_{x^{\prime \prime} \in[0, \epsilon]^{p}} \partial^{*} D\left(x^{\prime \prime}\right)$. Note that $\mathcal{H}_{\epsilon}$ is defined by

$$
\begin{gathered}
A_{j}\left(x^{\prime \prime}\right)\left|z_{j}\right|^{2}-B_{j}\left(x^{\prime \prime}\right)\left(z_{j}^{2}+\bar{z}_{j}^{2}\right) \leq x_{p+j}, \quad 1 \leq j \leq p \\
y^{\prime \prime}=0, \quad x^{\prime \prime} \in[0, \epsilon]^{p}
\end{gathered}
$$

with

$$
A_{j}\left(x^{\prime \prime}\right)=\frac{1+\Lambda_{1 j}^{2}\left(R^{-1}\left(x^{\prime \prime}\right)\right)}{S_{j}\left(x^{\prime \prime}\right)\left(1-\Lambda_{1 j}^{2}\left(R^{-1}\left(x^{\prime \prime}\right)\right)^{2}\right.}, \quad B_{j}\left(x^{\prime \prime}\right)=\frac{\Lambda_{1 j}\left(R^{-1}\left(x^{\prime \prime}\right)\right)}{S_{j}\left(x^{\prime \prime}\right)\left(1-\Lambda_{1 j}^{2}\left(R^{-1}\left(x^{\prime \prime}\right)\right)^{2}\right.} .
$$

Let $\delta$ be a small positive number. For $x^{\prime \prime} \in[-\delta, \epsilon]^{p}$, let $D_{j}^{\delta}\left(x^{\prime \prime}\right) \subset \mathbf{C}$ be defined by

$$
A_{j}\left(x^{\prime \prime}\right)\left|z_{j}\right|^{2}-B_{j}\left(x^{\prime \prime}\right)\left(z_{j}^{2}+\bar{z}_{j}^{2}\right) \leq x_{p+j}+\delta .
$$

Let $P_{\epsilon}^{\delta}\left(\right.$ resp. $\left.\partial^{*} P_{\epsilon}^{\delta}\right)$ be the set of $z=\left(z^{\prime}, z^{\prime \prime}\right)$ such that $y^{\prime \prime} \in[-\delta, \delta]^{p}, x^{\prime \prime} \in[-\delta, \epsilon]^{p}$, and $z_{j} \in D_{j}^{\delta}\left(x^{\prime \prime}\right)$ (resp. $\left.z_{j} \in \partial D_{j}^{\delta}\left(x^{\prime \prime}\right)\right)$ for $1 \leq j \leq p$. Let $\mathcal{U}_{\epsilon}^{\delta}$ (resp. $\mathcal{U}_{\epsilon}^{\delta_{1}}$ ) be a small neighborhood of $P_{\epsilon}^{\delta}$ (resp. $P_{\epsilon}^{\delta_{1}}$ ). Assume that $0<\delta_{1}<\delta$ and $\delta_{1}$ is sufficiently small. We may also assume that $\mathcal{U}_{\epsilon}^{\delta_{1}}$ is contained in $\mathcal{U}_{\epsilon}^{\delta}$ and $\partial^{*} P_{\epsilon}^{\delta} \subset \mathcal{U}$. Thus, for $\left(z^{\prime}, z^{\prime \prime}\right) \in \mathcal{U}_{\epsilon}^{\delta_{1}}$, we can define

$$
F\left(z^{\prime}, z^{\prime \prime}\right)=\frac{1}{(2 \pi i)^{p}} \int_{\zeta_{1} \in \partial D_{1}^{\delta}\left(x^{\prime \prime}\right)} \cdots \int_{\zeta_{p} \in \partial D_{p}^{\delta}\left(x^{\prime \prime}\right)} \frac{f\left(\zeta, z^{\prime \prime}\right) d \zeta_{1} \cdots d \zeta_{p}}{\left(\zeta_{1}-z_{1}\right) \cdots\left(\zeta_{p}-z_{p}\right)}
$$

When $z$ is sufficiently small, $F(z)=f(z)$ as $f$ is holomorphic near the origin. Fix $z_{0} \in \mathcal{U}_{\epsilon}^{\delta_{1}}$. We want to show that $F$ is holomorphic at $z_{0}$. So $F$ is a desired extension of $f$. By continuity, when $z=\left(z_{1}, \ldots, z_{2 p}\right)$ tends to $z_{0}, x^{\prime \prime}$ tends to $x_{0}^{\prime \prime}$ and $\partial D_{j}^{\delta}\left(x^{\prime \prime}\right)$ tends to $\partial D_{j}^{\delta}\left(x_{0}^{\prime \prime}\right)$, while $z_{j} \in D_{j}^{\delta}\left(x_{0}^{\prime \prime}\right)$ when $z$ is sufficiently close to $z_{0}$. By Cauchy theorem, for $z$ sufficiently close to $z_{0}$ we change the repeated integral for $\zeta_{j} \in \partial D_{j}^{\delta}\left(x_{0}^{\prime \prime}\right), 1 \leq j \leq p$. The domain of integration is thus fixed. The integrand is holomorphic in $z$. Hence $F$ is holomorphic at $z=z_{0}$.

Next we want to show that $\mathcal{H}_{\epsilon}$ is the hull of holomorphy of $M_{\epsilon}$ in $B_{\epsilon_{0}}^{2 p}$ for suitable $\epsilon, \epsilon_{0}$ that can be arbitrarily small.

Let us first show that $\mathcal{H}_{\epsilon}$ is the intersection of domains of holomorphy in $\mathbf{C}^{2 p}$. Recall that $\mathcal{H}_{\epsilon}$ is defined by (5.20)-(5.21). Define for $\delta^{\prime}:=\left(\delta_{1}, \ldots, \delta_{p}\right)$ with $\delta_{j}>0$

$$
\begin{aligned}
\rho_{j}^{\delta^{\prime}}= & A_{j}\left(x^{\prime \prime}\right)\left|z_{j}\right|^{2}-B_{j}\left(x^{\prime \prime}\right)\left(z_{j}^{2}+\bar{z}_{j}^{2}\right)-x_{p+j}+\left(\delta_{1}^{-1}+\cdots+\delta_{p}^{-1}\right) \sum_{i=1}^{p} y_{p+i}^{2} \\
& +\sum_{i \neq j} \delta_{i}^{-1}\left\{A_{i}\left(x^{\prime \prime}\right)\left|z_{i}\right|^{2}-B_{i}\left(x^{\prime \prime}\right)\left(z_{i}^{2}+\bar{z}_{i}^{2}\right)-x_{p+i}\right\} .
\end{aligned}
$$


When $p=1$, the last summation is 0 . The complex Hessian of $\rho_{j}^{\delta^{\prime}}$ is

$$
\begin{aligned}
& \sum_{\alpha, \beta=1}^{2 p} \frac{\partial^{2} \rho_{j}^{\delta^{\prime}}}{\partial z_{\alpha} \bar{z}_{\beta}} t_{\alpha} \bar{t}_{\beta}=A_{j}\left(x^{\prime \prime}\right)\left|t_{j}\right|^{2}+\frac{\delta_{1}^{-1}+\cdots+\delta_{p}^{-1}}{2} \sum_{i}\left|t_{p+i}\right|^{2}+\sum_{i \neq j} \frac{1}{\delta_{i}} A_{i}\left(x^{\prime \prime}\right)\left|t_{i}\right|^{2} \\
& \quad+\operatorname{Re} \sum_{k} a_{j k}\left(x^{\prime \prime} ; z_{j}\right) t_{j} \bar{t}_{p+k}+\sum_{k, \ell} b_{j, k \ell}\left(x^{\prime \prime} ; z_{j}\right) t_{p+k} \bar{t}_{p+\ell} \\
& \quad+\operatorname{Re} \sum_{i \neq j} \sum_{k} \frac{1}{\delta_{i}} c_{j, i k}\left(x^{\prime \prime} ; z_{i}\right) t_{i} \bar{t}_{p+k}+\sum_{i \neq j} \sum_{k, \ell} \frac{1}{\delta_{i}} d_{j, k \ell}\left(x^{\prime \prime} ; z_{i}\right) t_{p+k} \bar{t}_{p+\ell} .
\end{aligned}
$$

Here $a_{j k}\left(x^{\prime \prime} ; 0\right)=b_{j, k l}\left(x^{\prime \prime} ; 0\right)=c_{j, i k}\left(x^{\prime \prime} ; 0\right)=d_{j, k l}\left(x^{\prime \prime} ; 0\right)=0$, and $i, j, k, \ell$ are in $\{1, \ldots, p\}$. From the Cauchy-Schwarz inequality, it follows that for $z \in B_{e_{0}}^{2 p}$ with $\epsilon_{0}>0$ sufficiently small and $0<\delta_{j}<1$,

$$
2 \sum_{\alpha, \beta=1}^{2 p} \frac{\partial^{2} \rho_{j}^{\delta^{\prime}}}{\partial z_{\alpha} \bar{z}_{\beta}} t_{\alpha} \bar{t}_{\beta} \geq A_{j}\left(x^{\prime \prime}\right)\left|t_{j}\right|^{2}+\frac{\delta_{1}^{-1}+\cdots+\delta_{p}^{-1}}{2} \sum_{j}\left|t_{p+j}\right|^{2}+\sum_{i \neq j} \delta_{i}^{-1} A_{i}\left(x^{\prime \prime}\right)\left|t_{i}\right|^{2} .
$$

Therefore, each $\rho_{j}^{\delta^{\prime}}$ is strictly plurisubharmonic on $|z|<\epsilon_{0}$ for all $0<\delta_{i}<1$. Hence for $\delta_{*}=\left(\delta_{0}, \ldots, \delta_{p}\right)=\left(\delta_{0}, \delta^{\prime}\right) \in(0,1)^{p+1}$,

$$
\rho_{\epsilon}^{\delta_{*}}(z)=\max _{j}\left\{\rho_{j}^{\delta^{\prime}},\left|y^{\prime \prime}\right|^{2}-\delta_{0}^{2}, x_{p+j}^{2}-\epsilon^{2}\right\}
$$

is plurisubharmonic on $B_{\epsilon_{0}}^{2 p}$. By (5.19), $D\left(x^{\prime \prime}\right)$ is contained in $B_{C_{2} \epsilon^{1 / 2}}^{2 p}$ for $x^{\prime \prime} \in[0, \epsilon]^{p}$. We now fix $\epsilon<\left(\epsilon_{0} / C_{2}\right)^{2}$ to ensure

$$
D\left(x^{\prime \prime}\right) \subset B_{\epsilon_{0}}^{2 p}, \quad \forall x^{\prime \prime} \in[0, \epsilon]^{p} .
$$

This shows that $\mathcal{H}_{\epsilon}^{\delta_{*}}:=\left\{z \in B_{\epsilon_{0}}^{2 p} \mid: \rho_{\epsilon}^{\delta_{*}}(z)<0\right\}$ is a domain of holomorphy.

Let us verify that $\mathcal{H}_{\epsilon}=\bigcap_{\epsilon^{\prime}>\epsilon, \delta_{0}>0, \ldots, \delta_{p}>0} \mathcal{H}_{\epsilon^{\prime}}^{\delta_{*}}$. Fix $z \in \mathcal{H}_{\epsilon}$. From (5.23) we get $z \in B_{\epsilon_{0}}^{2 p}$. We have $y^{\prime \prime}=0$. Hence (5.20) hold and $x_{p+j}^{2} \leq \epsilon^{2}$. Clearly, $\rho_{j}^{\delta}(z)<0$ for each $j$ and $\delta \in(0,1)^{p}$. This shows that $z \in \mathcal{H}_{\epsilon}$ is in the intersection. For the other inclusion, let us assume that $z$ is in the intersection. Then $y^{\prime \prime}=0$. With $\rho_{j}^{\delta_{*}}(z)<0$, we let $\delta_{i}$ tend to 0 for $i \neq j$. We conclude

$$
A_{i}\left(x^{\prime \prime}\right)\left|z_{i}\right|^{2}-B_{i}\left(x^{\prime \prime}\right)\left(z_{i}^{2}+\bar{z}_{i}^{2}\right) \leq x_{p+i}
$$

for all $i \neq j$, and hence for all $i$ as $p>1$. When $p=1$ the above inequality can be obtained directly from $\rho_{1}^{\delta_{*}}$. We also see that $0 \leq x_{p+j} \leq \epsilon$. We have verified (5.20) and (5.21). This shows that $z \in \mathcal{H}_{\epsilon}$. by

In view of (5.20)-(5.21), the boundary of $\mathcal{H}_{\epsilon}$ is the union $\cup_{j=1}^{p} \mathcal{H}_{j}^{\epsilon}$ with $\mathcal{H}_{j}^{\epsilon}$ being defined

$$
\begin{aligned}
A_{j}\left(x^{\prime \prime}\right)\left|z_{j}\right|^{2}-B_{j}\left(x^{\prime \prime}\right)\left(z_{j}^{2}+\bar{z}_{j}^{2}\right) & =x_{p+j}, \\
A_{i}\left(x^{\prime \prime}\right)\left|z_{i}\right|^{2}-B_{i}\left(x^{\prime \prime}\right)\left(z_{i}^{2}+\bar{z}_{i}^{2}\right) & \leq x_{p+i}, \quad 1 \leq i \leq p, \quad i \neq j ; \\
y^{\prime \prime}=0, \quad x_{p+i} & \leq \epsilon, \quad 1 \leq i \leq p .
\end{aligned}
$$

Therefore, we have proved the following theorem. 
Theorem 5.5. Let $M$ be a germ of real analytic submanifold at an abelian $C R$ singularity. Assume that the complex tangent of $M$ is purely elliptic at the origin. There is a base of neighborhoods $\left\{U_{j}\right\}$ of the origin in $\mathbf{C}^{n}$ which satisfies the following: For each $U_{j}$, the hull of holomorphy $H\left(M \cap U_{j}\right)$ of $M \cap U_{j}$ is foliated by embedded complex submanifolds with boundaries. Furthermore, near the origin $H\left(M \cap U_{j}\right)$ is the transversal intersection of $p$ real analytic submanifolds of dimension $3 p$ with boundary. The boundary of $H\left(M \cap U_{j}\right)$ contains $M \cap U_{j}$; and two sets are the same if and only if $p=1$.

Remark 5.6. The proof shows that the hull $H\left(M \cap U_{j}\right)$ is foliated by analytic polydiscs, i.e. holomorphic embeddings of closed unit polydisc in $\mathbf{C}^{p}$.

\section{RIGIDITY OF PRODUCT QUADRICS}

The aim of this section is to prove the following rigidity theorem: Let us consider a higher order analytic perturbation of a product quadric. If this manifold is formally equivalent to the product quadric, then under a small divisors condition, it is also holomorphically equivalent to it. Notice that when $p>1$, there are real submanifold $M$ with a linearizable $\sigma$ such that $M$ is not formally equivalent to the quadric, or equivalently, the $\left\{\tau_{1 j}, \rho\right\}$ is not formally linearizable; see GS15].

The proof goes as follows: Since the manifold is formally equivalent to the quadric, the associated involutions $\left\{\tau_{1 i}\right\}$ and $\left\{\tau_{2 i}\right\}$ are simultaneously linearizable by a formal biholomorphism that commutes with $\rho$. In particular, $\sigma_{1}, \ldots, \sigma_{p}$, as defined by (5.1) and (5.2), are formally linearizable and they commute pairwise. These are germs of biholomorphisms with a diagonal linear part. According to [Sto15] [theorem 2.1], this abelian family can be holomorphically linearized under a collective Brjuno type condition (7.38). Furthermore, the transformation commutes with $\rho$. Then, we linearize simultaneously and holomorphically both $\tau_{1}:=\tau_{11} \circ \cdots \circ \tau_{1 p}$ and $\tau_{2}:=\tau_{21} \circ \cdots \circ \tau_{2 p}$ by a transformation that commutes with both $\rho$ and $\mathcal{S}$, the family of linear parts of the $\sigma_{1}, \ldots, \sigma_{p}$. Finally, we linearize simultaneously and holomorphically both families $\left\{\tau_{1 i}\right\}$ and $\left\{\tau_{2 i}\right\}$ by a transformation that commutes with $\rho, \mathcal{S}, T_{1}$ and $T_{2}$.

These last two steps will be obtained through a majorant method and the application of a holomorphic implicit function theorem. This is obtained in Proposition 6.6. They first require a complete description of the various centralizers and their associated normalized mappings, i.e. suitable complements. This is a goal of Proposition 6.3.

Throughout this section, we do not assume that $\mu_{1}, \ldots, \mu_{p}$ are non resonant in the sense that $\mu^{Q} \neq 1$ if $Q \in \mathbf{Z}^{p}$ and $Q \neq 0$. In fact, we will apply our results to $M$ which might be resonant. However, we will retain the assumption that $\sigma$ has distinct eigenvalues when we apply the results to the manifolds.

6.1. Centralizers. We recall from (5.1) and (5.2), the definition and property of germs of holomorphic diffeomorphisms : $\sigma_{i}:=\tau_{1 i} \circ \tau_{2 i}, \sigma_{i}^{-1}=\rho \sigma_{i} \rho, 1 \leq i \leq e_{*}+h_{*} ; \sigma_{s}:=\tau_{1 s} \circ \tau_{2\left(s_{*}+s\right)}$, $\sigma_{s+s_{*}}:=\tau_{1\left(s+s_{*}\right)} \circ \tau_{2 s}, \sigma_{s+s_{*}}^{-1}=\rho \sigma_{s} \rho, e_{*}+h_{*}<s \leq p-s_{*}$. Recall the linear maps

$$
\begin{aligned}
& S: \xi_{j}^{\prime}=\mu_{j} \xi_{j}, \quad \eta_{j}^{\prime}=\mu_{j}^{-1} \eta_{j} ; \\
& S_{j}: \xi_{j}^{\prime}=\mu_{j} \xi_{j}, \quad \eta_{j}^{\prime}=\mu_{j}^{-1} \eta_{j}, \quad \xi_{k}^{\prime}=\xi_{k}, \quad \eta_{k}^{\prime}=\eta_{k}, \quad k \neq j ; \\
& T_{i j}: \xi_{j}^{\prime}=\lambda_{i j} \eta_{j}, \quad \eta_{j}^{\prime}=\lambda_{i j}^{-1} \xi_{j}, \quad \xi_{k}^{\prime}=\xi_{k}, \quad \eta_{k}^{\prime}=\eta_{k}, \quad k \neq j ;
\end{aligned}
$$


while $\rho$ is given by

$$
\rho:\left\{\begin{array}{l}
\left(\xi_{e}^{\prime}, \eta_{e}^{\prime}, \xi_{h}^{\prime}, \eta_{h}^{\prime}\right)=\left(\bar{\eta}_{e}, \bar{\xi}_{e}, \bar{\xi}_{h}, \bar{\eta}_{h}\right), \\
\left(\xi_{s}^{\prime}, \xi_{s+s_{*}}^{\prime}, \eta_{s}^{\prime}, \eta_{s+s_{*}}^{\prime}\right)=\left(\bar{\xi}_{s+s_{*}}, \bar{\xi}_{s}, \bar{\eta}_{s+s_{*}}, \bar{\eta}_{s}\right) .
\end{array}\right.
$$

Recall that indices $e . h, s$ have the ranges $1 \leq e \leq e_{*}, e_{*}<h \leq e_{*}+h_{*}$, and $e_{*}+h_{*}<s \leq$ $p-s_{*}$. The basic conditions on $\mu_{j}=\lambda_{j}^{2}$ are the following:

$$
\left|\mu_{h}\right|=1, \quad \mu_{s+s_{*}}=\bar{\mu}_{s}^{-1}, \quad \mu_{e}>1, \quad\left|\mu_{s}\right| \geq 1, \quad \mu_{s}^{k} \neq 1, k=1,2, \ldots
$$

In particular, a $\mu_{j}$ may be repeated and $\mu_{h}$ can be 1 .

We need to introduce notation for the indices to describe various centralizers regarding $T_{1 j}, S_{j}$ and $\rho$. We first introduce index sets for the centralizer of $\mathcal{S}:=\left\{S_{1}, \ldots, S_{p}\right\}, T_{1}:=$ $T_{i 1} \circ \cdots \circ T_{i p}, \rho$. We recall that $\mathcal{T}_{i}:=\left\{T_{i 1}, \ldots, T_{i p}\right\}$.

Let $(P, Q) \in \mathbf{N}^{p} \times \mathbf{N}^{p}$ and $1 \leq j \leq p$. By definition, $\xi^{P} \eta^{Q} e_{j}$ belongs to the centralizer of $\mathcal{S}$ if and only if it commutes with each $S_{i}$. In other words, $\xi^{P} \eta^{Q} e_{j} \in \mathcal{C}(\mathcal{S})$ if and only if

$$
\mu_{k}^{p_{k}-q_{k}}=1, \quad \forall k \neq j ; \quad \mu_{j}^{p_{j}-q_{j}}=\mu_{j} .
$$

Note that the same condition holds for $\xi^{Q} \eta^{P} e_{p+j}$ to belong to $\mathcal{C}(\mathcal{S})$. This leads us to define the set of multiindices

$$
\mathcal{R}_{j}:=\left\{(P, Q) \in \mathbf{N}^{2 p}: \mu_{j}^{p_{j}-q_{j}}=\mu_{j}, \mu_{i}^{p_{i}-q_{i}}=1, \forall i \neq j\right\}, \quad 1 \leq j \leq p .
$$

We observe that if $(P, Q) \in \mathcal{R}_{j}$, then (6.4) implies that

$$
\begin{gathered}
p_{j}=q_{j}+1, \quad j \neq h ; \quad p_{i}=q_{i}, \quad \forall i \neq j, h ; \\
\lambda_{h}^{p_{h}-q_{h}}= \pm 1, \quad h \neq j ; \quad \lambda_{j}^{p_{j}-q_{j}-1}= \pm 1, \quad j=h .
\end{gathered}
$$

Here we have used the assumption that $\mu_{s}$ are not root of unity, which simplifies greatly the results and computation in this section.

For convenience, we define for $P=\left(p_{e}, p_{h}, p_{s}, p_{s+s_{*}}\right)$ and $Q=\left(q_{e}, q_{h}, q_{s}, q_{s+s_{*}}\right)$

$$
\begin{aligned}
\rho(P Q) & :=\left(q_{e}, p_{h}, p_{s+s_{*}}, p_{s}, p_{e}, q_{h}, q_{s+s_{*}}, q_{s}\right), \\
\rho_{a}(P Q) & :=\left(q_{e}, p_{h}, p_{s+s_{*}}, p_{s}\right), \quad \rho_{b}(P Q):=\left(p_{e}, q_{h}, q_{s+s_{*}}, q_{s}\right), \\
\bar{f}_{\rho(P Q)} & :=(\overline{f \circ \rho})_{P Q} .
\end{aligned}
$$

Here $p_{e}=\left(p_{1}, \ldots, p_{e_{*}}\right)$ denotes the "elliptic coordinates" of $P$. Hence,

$$
\rho(P Q)=\left(\rho_{a}(P Q), \rho_{b}(P Q)\right)=\left(\rho_{b}(Q P), \rho_{a}(Q P)\right) .
$$

According to (6.5) and equation (6.3) of $\rho$, the restriction of $\rho$ to $\mathcal{R}_{h}$ is an involution, which will be denoted by $\rho_{h}$. Moreover, $\rho$ is a bijection $\rho_{s}$ from $\mathcal{R}_{s}$ onto $\mathcal{R}_{s+s_{*}}$. We define an involution on $\mathcal{R}_{e}$ by

$$
\rho_{e}(P Q):=\left(\rho_{b}(P Q), \rho_{a}(P Q)\right)=\left(p_{e}, q_{h}, q_{s+s_{*}}, q_{s}, q_{e}, p_{h}, p_{h}, p_{s+s_{*}}, p_{s}\right) .
$$

Note that $\rho_{e}$ is not a restriction of $\rho$, and $\rho_{s}$ is not an involution either.

Next, we introduce sets of indices to be used to compute the centralizers on $\mathcal{T}_{1}, \mathcal{T}_{2}, \rho$. Set

$$
\mathcal{N}_{j}:=\mathcal{R}_{j} \cap\left\{(P, Q): p_{i} \geq q_{i}, \quad \forall i \neq j\right\}, \quad 1 \leq j \leq p .
$$


Since there is no restriction for $p=1$, we have $\mathcal{N}_{j}=\mathcal{R}_{j}$ for $j=e$ or $h$. Let us set

$$
\begin{array}{rll}
A_{j k}(P, Q):=\max \left\{p_{k}, q_{k}\right\}, & k \neq j, & A_{j j}(P, Q)=p_{j} \\
B_{j k}(P, Q):=\min \left\{p_{k}, q_{k}\right\}, & k \neq j, & B_{j j}(P, Q)=q_{j} .
\end{array}
$$

We define a mapping

$$
\left(A_{j}, B_{j}\right): \mathcal{R}_{j} \rightarrow \mathcal{N}_{j}
$$

with $A_{j}:=\left(A_{j 1}, \ldots, A_{j p}\right)$ and $B_{j}:=\left(B_{j 1}, \ldots, B_{j p}\right)$. For $(P, Q) \in \mathcal{N}_{j}$ with $j=e, h$, we have $A_{j} \circ \rho_{j}(P, Q)=\left(p_{e}, p_{h}, p_{s+s_{*}}, p_{s}\right)$ and $B_{j} \circ \rho_{j}(P, Q)=\left(q_{e}, q_{h}, q_{s+s_{*}}, q_{s}\right)$. In other words, on $\mathcal{N}_{j}$ for $j=e$ or $h, A_{j} \circ \rho_{j}$ just interchanges the $s$ th and the $\left(s+s_{*}\right)$ th coordinates for each $s$, so does $B_{j} \circ \rho_{j}$, while $A_{s+s_{*}} \rho_{s}$ and $B_{s+s_{*}} \rho_{s}$ have the same property on $\mathcal{N}_{s}$. Furthermore,

$$
\begin{gathered}
\left(A_{h}, B_{h}\right) \rho=\rho\left(A_{h}, B_{h}\right) \quad \text { on } \mathcal{R}_{h}, \\
\left(A_{s+s_{*}}, B_{s+s_{*}}\right) \rho=\rho\left(A_{s}, B_{s}\right) \quad \text { on } \mathcal{R}_{s} .
\end{gathered}
$$

Finally, with the convention that the product over an empty set is 1 , we define, for $(P, Q) \in \mathcal{R}_{j}:$

$$
\begin{aligned}
& \nu_{P Q}:= \begin{cases}\prod_{h^{\prime}} \lambda_{h^{\prime}}^{q_{h^{\prime}}-p_{h^{\prime}},} & j \neq h, \\
\lambda_{h}^{p_{h}-q_{h}-1} \prod_{h^{\prime} \neq h} \lambda_{h^{\prime}}^{q_{h^{\prime}}-p_{h^{\prime}}}, & j=h ;\end{cases} \\
& \nu_{P Q}^{+}:= \begin{cases}\prod_{h^{\prime} \mid q_{h^{\prime}}>p_{h^{\prime}}} \lambda_{h^{\prime}}^{q_{h^{\prime}}-p_{h^{\prime}},}, & j \neq h ; \\
\prod_{h^{\prime} \neq h, q_{h^{\prime}}>p_{h^{\prime}}} \lambda_{h^{\prime}}^{q_{h^{\prime}}-p_{h^{\prime}},}, & j=h .\end{cases}
\end{aligned}
$$

Here $e_{*}<h^{\prime}, h \leq e_{*}+h_{*}$. For convenience, we however define

$$
\nu_{Q P}:=\nu_{P Q}, \quad(P, Q) \in \mathcal{R}_{j} .
$$

If $p=1$ we set $\nu_{P Q}^{+}=1$.

Lemma 6.1. Let $(P, Q) \in \mathcal{R}_{j}$. Then $\lambda_{j}^{-1} \lambda^{P-Q}=\nu_{P Q}$, and

$$
\begin{array}{ll}
\nu_{P Q}= \pm 1 ; \quad \nu_{P Q}^{+}= \pm 1 ; & \nu_{P Q}^{+}=1, \quad(P, Q) \in \mathcal{N}_{j} ; \\
\nu_{\rho_{e}(P Q)}=\nu_{P Q}, j=e ; & \nu_{\rho(P Q)}=\nu_{P Q} ; \\
\nu_{\rho_{e}(P Q)}^{+}=\nu_{P Q}^{+} \nu_{P Q}, j=e ; & \nu_{\rho(P Q)}^{+}=\nu_{P Q}^{+} .
\end{array}
$$

Proof. The first identity follows from the definition of $\nu_{P Q}$ and $\mathcal{R}_{j}$. From the definition of $\mathcal{R}_{j}$, we have $\left(\lambda_{i}^{p_{i}-q_{i}}\right)^{2}=\mu_{i}^{p_{i}-q_{i}}=1$ for $i=h^{\prime}$ in (6.13)-(6.14). We also have $\mu_{h}^{p_{h}-q_{h}-1}=1$ for terms in (6.13)-(6.14). Thus

$$
\lambda_{h^{\prime}}^{p_{h^{\prime}}-q_{h^{\prime}}}= \pm 1, \quad \lambda_{h}^{p_{h}-q_{h}-1}= \pm 1 .
$$

Thus we obtain (6.15); the rest identities follow from the definition of $\rho_{e}, \rho$, and the above identities. 
Lemma 6.2. For all multiindices $(P, Q), \xi^{P} \eta^{Q} \circ \rho=\bar{\xi}^{\rho_{a}(P Q)} \bar{\eta}^{\rho_{b}(P Q)}$. For all multiindices $(P, Q) \in \mathcal{R}_{e} \cup \mathcal{R}_{h}$, we have

$$
\begin{gathered}
\overline{\lambda^{\rho_{a}(P, Q)-\rho_{b}(P, Q)}}=\lambda^{Q-P}, \quad \overline{\mu^{\rho_{b}-\rho_{a}}}=\mu^{P-Q}, \\
\xi^{P} \eta^{Q} \circ \rho \circ T_{1}=\lambda^{Q-P} \bar{\xi}^{\rho_{b}(P Q)} \bar{\eta}^{\rho_{a}(P Q)}, \\
\xi^{P} \eta^{Q} \circ \rho \circ S^{-1}=\mu^{P-Q} \bar{\xi}^{\rho_{a}(P Q)} \bar{\eta}^{\rho_{b}(P Q)} .
\end{gathered}
$$

Proof. Identity (6.18) follows from (6.7) and the fact that $\lambda_{e}$ and $\mu_{e}$ are reals, $\lambda_{h}^{-1}=\bar{\lambda}_{h}$, $p_{s}=q_{s}$, and $p_{s+s_{*}}=q_{s+s_{*}}$. A direct computation shows that

$$
\xi^{P} \eta^{Q} \circ \rho \circ T_{1}=\bar{\lambda}^{\rho_{a}-\rho_{b}} \bar{\xi}^{\rho_{b}(P Q)} \bar{\eta}^{\rho_{a}(P Q)}, \quad \xi^{P} \eta^{Q} \circ \rho \circ S^{-1}=\bar{\mu}^{\rho_{b}-\rho_{a}} \bar{\xi}^{\rho_{a}(P Q)} \bar{\eta}^{\rho_{b}(P Q)} .
$$

The result follows from (6.18).

Finally we note that

$$
\iota_{e}:(P, Q) \rightarrow\left(A_{e}, B_{e}\right) \circ \rho_{e}(P Q)=\left(A_{e}, B_{e}\right)\left(\rho_{b}(P, Q), \rho_{a}(P, Q)\right)
$$

defines an involution on $\mathcal{N}_{e}$. We now can describe the centralizers.

Proposition 6.3. Let $\mathcal{S}=\left\{S_{1}, \ldots, S_{p}\right\}, \mathcal{T}_{i}=\left\{T_{i 1}, \ldots, T_{i p}\right\}$ and $\rho$ be given by (6.1) -(6.3). Let $\varphi=I+(U, V)$ be a formal biholomorphic map that is tangent to the identity.

(i) $\varphi \in \mathcal{C}(\mathcal{S})$ if and only if

$$
U_{j, P Q}=0=V_{j, Q P}, \quad \forall(P, Q) \notin \mathcal{R}_{j} .
$$

Also, $\varphi \in \mathcal{C}(\mathcal{S}, \rho)$ if and only if (6.22) holds and

$$
\begin{aligned}
U_{h, P Q}=\bar{U}_{h, \rho(P Q)},(P, Q) \in \mathcal{R}_{h} ; \quad U_{s+s_{*}, P Q} & =\bar{U}_{s, \rho(P Q)},(P, Q) \in \mathcal{R}_{s+s_{*}} ; \\
V_{e, Q P}=\bar{U}_{e, \rho_{e}(P Q)}, \quad(P, Q) & \in \mathcal{R}_{e} ; \\
V_{h, Q P}=\bar{V}_{h, \rho(Q P)}, \quad(P, Q) \in \mathcal{R}_{h} ; \quad V_{s+s_{*}, Q P} & =\bar{V}_{s, \rho(Q P)}, \quad(P, Q) \in \mathcal{R}_{s+s_{*}} .
\end{aligned}
$$

(ii) $\varphi \in \mathcal{C}\left(\mathcal{S}, T_{1}\right)$ if and only if (6.22) holds and

$$
V_{j, Q P}=\nu_{P Q} U_{j, P Q}, \quad \forall(P, Q) \in \mathcal{R}_{j} .
$$

(iii) $\varphi \in \mathcal{C}\left(\mathcal{S}, T_{1}, \rho\right)$ if and only if (6.22), (6.23) and (6.26) hold, and

$$
U_{e, P Q}=\nu_{P Q} \bar{U}_{e, \rho_{e}(P Q)}, \quad(P, Q) \in \mathcal{R}_{e} .
$$

(iv) Let $p>1 . \varphi \in \mathcal{C}\left(\mathcal{T}_{1}, \mathcal{T}_{2}\right)$ if and only if (6.22) and (6.26) hold, and

$$
U_{j, P Q}=\nu_{P Q}^{+} U_{j,\left(A_{j}, B_{j}\right)(P, Q)}, \quad(P, Q) \in \mathcal{R}_{j} \backslash \mathcal{N}_{j} .
$$

Also, $\varphi \in \mathcal{C}\left(\mathcal{T}_{1}, \mathcal{T}_{2}, \rho\right)$ if and only if (6.22), (6.26) and (6.28) hold, and

$$
\begin{array}{rlrl}
U_{e, P Q} & =\bar{U}_{e,\left(A_{e}, B_{e}\right) \rho_{e}(P Q),} & (P, Q) \in \mathcal{N}_{e}, \\
U_{h, P Q} & =\bar{U}_{h, \rho(P Q)}, & & (P, Q) \in \mathcal{N}_{h}, \\
U_{s+s_{*}, P Q} & =\bar{U}_{s, \rho(P Q),} & & (P, Q) \in \mathcal{N}_{s+s_{*}} .
\end{array}
$$

We remark that condition (6.28) holds trivially when $(P, Q) \in \mathcal{N}_{j}$, in which case it becomes $U_{j, P Q}=U_{j, P Q}$. 
Proof. To simplify notation, we abbreviate

$$
\rho_{a}=\rho_{a}(P Q), \quad \rho_{b}=\rho_{b}(P Q), \quad A_{j}=A_{j}(P, Q), \quad B_{j}=B_{j}(P, Q) .
$$

Recall that $\lambda_{e}=\bar{\lambda}_{e}, \lambda_{h}=\bar{\lambda}_{h}^{-1}$ and $\lambda_{s+s_{*}}=\bar{\lambda}_{s}^{-1}$. By definition,

$$
S_{e}=T_{1 e} T_{2 e}, \quad S_{h}=T_{1 h} T_{2 h}, \quad S_{s}=T_{1 s} T_{2\left(s+s_{*}\right)}, \quad S_{s+s_{*}}=T_{1\left(s+s_{*}\right)} T_{2 s} .
$$

In the proof, we will use the fact that $S_{j}$ is reversible by both involutions in the composition for $S_{j}$. In particular,

$$
T_{1 j} S_{j} T_{1 j}=S_{j}^{-1}, \quad \forall j .
$$

However, we have $T_{2\left(s+s_{*}\right)} S_{s} T_{2\left(s+s_{*}\right)}=S_{s}^{-1}$ and $T_{2 s} S_{s+s_{*}} T_{2 s}=S_{s+s_{*}}^{-1}$. For simplicity, we will derive identities by using (6.32) and

$$
S_{e}^{-1}=\rho S_{e} \rho, \quad S_{h}^{-1}=\rho S_{h} \rho, \quad S_{s+s_{*}}^{-1}=\rho S_{s} \rho .
$$

Finally, we need one more identity. Recall that

$$
\begin{array}{llll}
T_{1 e} T_{2 j}=T_{2 j} T_{1 e}, & j \neq e ; & T_{1 h} T_{2 j}=T_{2 j} T_{1 h}, \quad j \neq h ; \\
T_{1 s} T_{2 j}=T_{2 j} T_{1 s}, & j \neq s+s_{*} ; & T_{1\left(s+s_{*}\right)} T_{2 j}=T_{2 j} T_{1\left(s+s_{*}\right)}, \quad j \neq s .
\end{array}
$$

Therefore, for any $j$ we have the identity

$$
T_{1} S_{j} T_{1}=S_{j}^{-1}
$$

In what follows, we will derive all identities by using (6.32), (6.33) and (6.34), as well as $S_{i} S_{j}=S_{j} S_{i}, T_{1 i} T_{1 j}=T_{1 j} T_{1 i}$ and $T_{2}=\rho T_{1} \rho$.

(i) The centralizer of $\mathcal{S}$ is easy to describe. Namely, $\varphi \in \mathcal{C}(\mathcal{S})$ if and only if

$$
\begin{gathered}
U_{j} \circ S_{j}=\mu_{j} U_{j}, \quad U_{j} \circ S_{k}=U_{j}, \quad k \neq j, \\
V_{j} \circ S_{j}=\mu_{j}^{-1} V_{j}, \quad V_{j} \circ S_{k}=V_{j}, \quad k \neq j .
\end{gathered}
$$

For $\varphi \rho=\rho \varphi$, we need

$$
\begin{gathered}
U_{h}=\overline{U_{h} \circ \rho}, \quad U_{s+s_{*}}=\overline{U_{s} \circ \rho}, \\
V_{e}=\overline{U_{e} \circ \rho}, \quad V_{h}=\overline{V_{h} \circ \rho}, \quad V_{s+s_{*}}=\overline{V_{s} \circ \rho} .
\end{gathered}
$$

Hence, using (6.8)-(6.10), we have $\bar{U}_{e, P Q}=V_{e, \rho(P Q)}=V_{e, \rho_{e}(Q P)}$. The other equalities are obtained in the same way.

(ii) If $\varphi \in \mathcal{C}\left(\mathcal{S}, T_{1}\right) \subset \mathcal{C}\left(S, T_{1}\right)$, then it satisfies

$$
V_{j}=\lambda_{j}^{-1} U_{j} \circ T_{1}
$$

This implies (6.26).

(iii) Assume furthermore that $\varphi \in \mathcal{C}\left(\mathcal{S}, T_{1}, \rho\right)$. Eliminating $V_{e}$ from (6.37) and (6.36), we obtain

$$
U_{e}=\lambda_{e} \overline{U_{e} \circ \rho \circ T_{1}} .
$$

According to (6.19), we obtain (6.27) by

$$
U_{e, \rho_{b} \rho_{a}}=\lambda_{e} \bar{\lambda}^{Q-P} \bar{U}_{e, P Q}=\nu_{P Q} \bar{U}_{e, P Q}
$$

(iv) Let $\varphi \in \mathcal{C}\left(\mathcal{T}_{1}, \mathcal{T}_{2}\right)$. Then, in particular, we have

$$
U_{j}=U_{j}\left(T_{1 k}\right), \quad k \neq j ; \quad V_{j}=\lambda_{j}^{-1} U_{j} \circ T_{1} .
$$


Let $(P, Q) \in \mathcal{R}_{j} \backslash \mathcal{N}_{j}$. We compose $U_{j}$ successively by each $T_{1 k}$ if $q_{k}>p_{k}$. We emphasize that such a $k$ is a hyperbolic index. The previous identity yields

$$
U_{j, P Q}=L_{j, P Q} U_{j, A_{j} B_{j}}, \quad L_{j, P Q}:=\prod_{k \neq j, p_{k}<q_{k}} \lambda_{k}^{q_{k}-p_{k}} .
$$

By the definition of $\nu_{P Q}^{+}$, we conclude

$$
L_{j, P Q}=\nu_{P Q}^{+}, \quad(P, Q) \in \mathcal{R}_{j} .
$$

If $(P, Q) \in \mathcal{N}_{j}$, then $\left(A_{j}, B_{j}\right)=(P, Q)$ and we have $L_{j, P Q}=\nu_{P Q}^{+}=1$, so that the relation (6.38) just becomes the identity $U_{j, P Q}=U_{j, P Q}$.

Assume now that $\varphi \in \mathcal{C}\left(\mathcal{T}_{1}, \mathcal{T}_{2}, \rho\right)$. In addition to the previous conditions, we have (6.35) and (6.36). Hence, (6.23), (6.27) and (6.38) lead to:

$$
\begin{aligned}
\nu_{P Q} \bar{U}_{e, \rho_{e}(P Q)} & =U_{e, P Q}=L_{e, P Q} U_{e, A_{e} B_{e}}, & & (P, Q) \in \mathcal{R}_{e} ; \\
\bar{U}_{h, \rho_{h}(P Q)} & =U_{h, P Q}=L_{h, P Q} U_{h, A_{h} B_{h}}, & & (P, Q) \in \mathcal{R}_{h} \\
\bar{U}_{s+s_{*}, \rho(P Q)} & =U_{s, P Q}=L_{s, P Q} U_{s, A_{s} B_{s}}, & & (P, Q) \in \mathcal{R}_{s} .
\end{aligned}
$$

Since $\rho_{e}, \rho_{h}$ are involutions on $\mathcal{R}_{e}$ and $\mathcal{R}_{h}$, respectively, and since $\rho$ is a bijection from $\mathcal{R}_{s}$ onto $\mathcal{R}_{s+s_{*}}$, we obtain

$$
\begin{aligned}
\nu_{\rho_{e}(P Q)} \bar{U}_{e, P Q} & =L_{e, \rho_{e}(P Q)} U_{e,\left(A_{e}, B_{e}\right) \circ \rho_{e}(P Q),} & & (P, Q) \in \mathcal{R}_{e} \\
\bar{U}_{h, P Q} & =L_{h, \rho_{h}(A B)} U_{h,\left(A_{h}, B_{h}\right) \circ \rho_{h}(P Q),} & & (P, Q) \in \mathcal{R}_{h} \\
\bar{U}_{s+s_{*}, P Q} & =L_{s, \rho(A B)} U_{s,\left(A_{s}, B_{s}\right) \circ \rho(P Q)}, & & (P, Q) \in \mathcal{R}_{s+s_{*}} .
\end{aligned}
$$

By (6.39), we copy the values $L_{j, \rho(P Q)}=\nu_{\rho(P Q)}^{+}$from (6.17). We have

$$
\begin{array}{ll}
\nu_{\rho_{j}(P Q)}^{+}=\nu_{P Q}^{+}, & \text {if } j \neq e, \text { and }(P, Q) \in \mathcal{R}_{j} ; \\
\nu_{\rho_{e}(P Q)}^{+}=\nu_{P Q} \nu_{P Q}^{+}, & \text {if }(P, Q) \in \mathcal{R}_{e} ; \\
\nu_{\rho_{e}(P Q)}=\nu_{P Q}, & \text { if }(P, Q) \in \mathcal{R}_{e} .
\end{array}
$$

Finally, we obtain

$$
\begin{aligned}
U_{j, P Q} & =\nu_{P Q}^{+} \bar{U}_{j,\left(A_{j}, B_{j}\right) \circ \rho_{j}(P Q)}, \quad(P, Q) \in \mathcal{R}_{j}, \quad j=e, h ; \\
U_{s+s_{*}, P Q} & =\nu_{P Q}^{+} \bar{U}_{s,\left(A_{s}, B_{s}\right) \circ \rho(P Q)}, \quad(P, Q) \in \mathcal{R}_{s+s_{*}} .
\end{aligned}
$$

Therefore, we have derived necessary conditions for the centralizers. Let us verify that the conditions are also sufficient. Of course, the verification for (i) is straightforward. Furthermore, that $\varphi=I+(U, V)$ commutes with $S_{1}, \ldots, S_{p}$ is equivalent to $U_{j, P Q}=$ $V_{j, Q P}=0$ for all $(P, Q) \in \mathcal{R}_{j}$, which is also trivial in cases (ii) and (iii).

For (ii), (6.22) and (6.26) imply that $\varphi$ commutes with $T_{1}$. We verify that $\varphi$ commutes with $\rho$. In other words, (6.24) and (6.25) hold. The latter follows immediately from (6.23) and (6.26). For the former, take $(P, Q) \in \mathcal{R}_{e}$. By (6.26) and (6.27), we get $V_{e, Q P}=$ $\nu_{P Q} U_{e, P Q}=\bar{U}_{e, \rho_{e}(P Q)}$, which is (6.24).

For (iii), let us verify that (6.28), (6.22), and (6.26) are sufficient conditions for $\varphi \in$ $\mathcal{C}\left(\mathcal{T}_{1}, \mathcal{T}_{2}\right)$. By (6.26), we get $\varphi T_{1}=T_{1} \varphi$. Also, for $\varphi \in \mathcal{C}\left(\mathcal{T}_{1}\right)$ it remains to show that for 
$(P, Q) \in \mathcal{R}_{j}$

$$
\left(U_{j} \circ T_{1 k}\right)_{P Q}=U_{j, P Q}, \quad k \neq j ; \quad\left(U_{j} \circ T_{1 j}\right)_{Q P}=\lambda_{j} V_{j, Q P} .
$$

We introduce $\left(P_{j}, Q_{j}\right)$ via $\xi^{P} \eta^{Q} \circ T_{1 j}=\lambda_{j}^{p_{j}-q_{j}} \xi^{P_{j}} \eta^{Q_{j}}$ and also denote $\left(P_{j}, Q_{j}\right)$ by $(P, Q)_{j}$. We remark that (6.28) also holds for $(P, Q) \in \mathcal{N}_{j}$. Therefore, we will use (6.28) for all $(P, Q) \in \mathcal{R}_{j}$.

For $k \neq j, h$, we have $\left(P_{k}, Q_{k}\right)=(P, Q)$. Thus in this case we immediately get the first identity in (6.40). Using (6.28) twice, we obtain for $j \neq h$

$$
\begin{aligned}
\left(U_{j} \circ T_{1 h}\right)_{P Q} & =\lambda_{h}^{p_{h}-q_{h}} U_{j,(P Q)_{h}}=\lambda_{h}^{p_{h}-q_{h}} \nu_{(P Q)_{h}}^{+} U_{j,\left(A_{j}, B_{j}\right)(P, Q)} \\
& =\lambda^{p_{h}-q_{h}} \nu_{(P Q)_{h}}^{+} \bar{\nu}_{P Q}^{+} U_{j, P Q}=U_{j, P Q} .
\end{aligned}
$$

Combining with the identities which we have proved, we get $\left(U_{j} \circ T_{1 j}\right)_{Q P}=\left(U_{j} \circ T_{1}\right)_{Q P}=$ $\left(\lambda_{j} V_{j}\right)_{Q P}$ for $j \neq h$. This gives us all the identities in (6.40) for $(P, Q) \in \mathcal{R}_{j}$. These identities are trivial when $(P, Q)$ is not in $\mathcal{R}_{j}$. Therefore, we have shown that these conditions are sufficient for $\varphi \in \mathcal{C}\left(\mathcal{T}_{1}, \mathcal{T}_{2}\right)$.

Finally, we need to verify that (6.22), (6.26), and (6.28)-(6.31) imply that $\varphi$ and $\rho$ commute. In other words, we need to verify (6.23) and (6.27), by (iii). We have

$$
\left(A_{e} B_{e}\right) \circ \rho_{e} \circ\left(A_{e} B_{e}\right)=\left(A_{e} B_{e}\right) \circ \rho_{e} \text { on } \mathcal{R}_{e} .
$$

Let $(P, Q) \in \mathcal{R}_{e}$. By (6.28), (6.29), (6.41) and (6.28), we get

$$
U_{e, P Q}=\nu_{P Q}^{+} U_{e,\left(A_{e}, B_{e}\right)(P, Q)}=\nu_{P Q}^{+} \bar{U}_{e,\left(A_{e}, B_{e}\right) \rho_{e}(P, Q)}=\nu_{P Q}^{+} \nu_{\rho_{e}(P, Q)}^{+} \bar{U}_{e, \rho_{e}(P, Q)} .
$$

By (6.17), (6.15), $\nu_{P Q}^{+} \nu_{\rho_{e}(P Q)}^{+}=\nu_{P Q}$. We obtain (6.27). Let us prove (6.23) with $P Q \in$ $\mathcal{R}_{s+s_{*}}$. Using (6.28), (6.31) with $P Q=\left(A_{s+s_{*}} B_{s+s_{*}}\right)(P Q)$, (6.12), (6.28) with $P Q=\rho(P Q)$ successively, we get

$$
\begin{aligned}
U_{s+s_{*}, P Q} & =\nu_{P Q}^{+} U_{s+s_{*}, A_{s+s_{*}} B_{s+s_{*}}(P Q)}=\nu_{P Q}^{+} \bar{U}_{s, \rho\left(A_{s+s_{*}} B_{s+s_{*}}(P Q)\right)} \\
& =\nu_{P Q}^{+} \bar{U}_{s, A_{s} B_{s}(\rho(P Q))}=\nu_{P Q}^{+} \nu_{\rho(P Q)}^{+} \bar{U}_{s, \rho(P Q) .}
\end{aligned}
$$

which gives us (6.23) by (6.17). To prove (6.23) for hyperbolic index, apply successively from left to right, (6.28), (6.30) with $P Q=\left(A_{h} B_{h}\right)(P Q)$, (6.11) and (6.28) with $P Q=$ $\rho_{h}(P Q)$ :

$$
\begin{aligned}
\nu_{P Q}^{+} U_{h, P Q} & =U_{h,\left(A_{h} B_{h}\right)(P Q)}=\bar{U}_{h, \rho_{h}\left(A_{h} B_{h}\right)(P Q)} \\
& =\bar{U}_{h,\left(A_{h} B_{h}\right) \rho_{h}(P Q)}=\nu_{\rho_{h}(P Q)}^{+} \bar{U}_{h, \rho_{h}(P Q)} .
\end{aligned}
$$

By (6.17) again, we obtain (6.23). The proof is complete.

6.2. Normalized mappings. We have described the conditions on centralizers. We now determine complements of these conditions to define normalized mappings.

Definition 6.4. Let $\varphi=I+(U, V)$ be a formal mapping tangent to the identity.

(i) We say that $\varphi$ is normalized with respect to $S_{1}, \ldots, S_{p}$ if

$$
U_{j, P Q}=0=V_{j, Q P}, \quad \text { if }(P, Q) \in \mathcal{R}_{j}, \quad \forall j .
$$

Furthermore, $\rho \varphi \rho$ is normalized w.r.t. $S_{1}, \ldots, S_{p}$ if and only if $\varphi$ is. 
(ii) We say that $\varphi$ is normalized w.r.t. $\left\{\mathcal{S}, T_{1}\right\}$, if

$$
V_{j, Q P}=-\nu_{P Q} U_{j, P Q}, \quad(P, Q) \in \mathcal{R}_{j} .
$$

(iii) We say that $\varphi$ is normalized w.r.t. $\left\{\mathcal{S}, T_{1}, \rho\right\}$ if

$$
\begin{aligned}
& U_{h, P Q}=-\bar{U}_{h, \rho(P Q)}, \quad \forall(P, Q) \in \mathcal{R}_{h} ; \\
& U_{s+s_{*}, P Q}=-\bar{U}_{s, \rho(P Q)}, \quad \forall(P, Q) \in \mathcal{R}_{s+s_{*}} \text {; } \\
& U_{e, P Q}=-\nu_{P Q} \bar{U}_{e, \rho_{e}(P Q)}, \quad \forall(P, Q) \in \mathcal{R}_{e} .
\end{aligned}
$$

(iv) Let $p>1$. We say that $\varphi$ is normalized w.r.t. $\left\{\mathcal{T}_{1}, \mathcal{T}_{2}\right\}$ if

$$
U_{j, P Q}=0, \quad(P, Q) \in \mathcal{N}_{j} .
$$

We say that $\varphi$ is normalized w.r.t. $\left\{\mathcal{T}_{1}, \mathcal{T}_{2}, \rho\right\}$ if

$$
\begin{aligned}
& U_{e, P Q}=-\bar{U}_{e,\left(A_{e}, B_{e}\right) \circ \rho_{e}(P, Q)}, \quad \forall(P, Q) \in \mathcal{N}_{e} ; \\
& U_{h, P Q}=-\bar{U}_{h, \rho(P, Q)}, \quad \forall(P, Q) \in \mathcal{N}_{h} ; \\
& U_{s+s_{*}, P Q}=-\bar{U}_{s, \rho(P, Q)}, \quad \forall(P, Q) \in \mathcal{N}_{s+s_{*}} .
\end{aligned}
$$

The set of normalized mapping w.r.t. to a family $\mathcal{F}$ is denoted $\mathcal{C}^{\mathrm{c}}(\mathcal{F})$.

Lemma 6.5. Let $F$ be a formal map which is tangent to the identity. There exists a unique formal decomposition $F=H G^{-1}$ with $G \in \mathcal{C}\left(\mathcal{S}, T_{1}, \rho\right)\left(\operatorname{resp} . \mathcal{C}\left(\mathcal{T}_{1}, \mathcal{T}_{2}, \rho\right)\right)$ and $H \in$ $\mathcal{C}^{\mathrm{c}}\left(\mathcal{S}, T_{1}, \rho\right)$ (resp. $\left.\left.\mathcal{C}^{\mathrm{c}}\left(\mathcal{T}_{1}, \mathcal{T}_{2}, \rho\right)\right)\right)$. If $F$ is convergent, then $G$ and $H$ are also convergent.

Proof. We will apply Lemma 4.3 as follows. Let $\hat{H}$ be the set of mappings in $\mathcal{C}_{2}^{\mathrm{c}}\left(\mathcal{S}, \mathcal{T}_{1}, \rho\right)$. Note that $\hat{H}$ is a $\mathbf{R}$-linear subspace of $\left(\widehat{\mathfrak{M}}_{n}^{2}\right)^{n}$. We will define a $\mathbf{R}$-linear projection $\pi$ from $\left(\widehat{\mathfrak{M}}_{n}^{2}\right)^{n}$ onto $\hat{H}$ such that $\pi$ preserves the degree of $F$ if $F$ is homogeneous. We will show that $\hat{G}=(\mathrm{I}-\pi) \hat{H}$ agrees with $\mathcal{C}_{2}\left(\mathcal{S}, \mathcal{T}_{1}, \rho\right)$. We will derive estimates on $\pi$ stated in Lemma 4.3, from which we conclude the convergence of $H, G$. The same argument will be applied to the second case of $\mathcal{C}\left(\mathcal{T}_{1}, \rho\right)$ and $\mathcal{C}^{\mathrm{c}}\left(\mathcal{T}_{1}, \rho\right)$.

For the first case, let us define a projection $\pi:\left(\widehat{\mathfrak{M}}_{n}^{2}\right)^{n} \rightarrow \hat{H}$. We decompose

$$
(U, V)=\left(U^{\prime}+U^{\prime \prime}, V^{\prime}+V^{\prime \prime}\right), \quad \pi(U, V)=\left(U^{\prime}, V^{\prime}\right) .
$$

We first define

$$
U_{j, P Q}^{\prime}=U_{j, P Q}, \quad V_{j, P Q}^{\prime}=V_{j, P Q}, \quad U_{j, P Q}^{\prime \prime}=0, \quad V_{j, P Q}^{\prime \prime}=0,
$$

for $(P, Q) \notin \mathcal{R}_{j}$. Suppose that $(P, Q) \in \mathcal{R}_{e}$. We have

$$
U_{e, P Q}=U_{e, P Q}^{\prime}+U_{e, P Q}^{\prime \prime}, \quad U_{e, \rho_{e}(P Q)}=U_{e, \rho_{e}(P Q)}^{\prime}+U_{e, \rho_{e}(P Q)}^{\prime \prime}
$$

According to (6.45) and (6.27), we need to seek solutions that satisfy

$$
U_{e, P Q}^{\prime}+\nu_{P Q} \bar{U}_{e, \rho_{e}(P Q)}^{\prime}=0, \quad U_{e, P Q}^{\prime \prime}-\nu_{P Q} \bar{U}_{e, \rho_{e}(P Q)}^{\prime \prime}=0 .
$$

Hence, for $(P, Q) \in \mathcal{R}_{e}$ we choose

$$
U_{e, P Q}^{\prime}=\frac{1}{2}\left(U_{e, P Q}-\nu_{P Q} \bar{U}_{e, \rho_{e}(P Q)}\right), U_{e, P Q}^{\prime \prime}=\frac{1}{2}\left(U_{e, P Q}+\nu_{P Q} \bar{U}_{e, \rho_{e}(P Q)}\right) .
$$


We verify directly that the solutions satisfy (6.51) as follows:

$$
\begin{aligned}
U_{e, P Q}^{\prime}+\nu_{P Q} \bar{U}_{e, \rho_{e}(P Q)}^{\prime}= & \frac{1}{2}\left(U_{e, P Q}-\nu_{P Q} \bar{U}_{e, \rho_{e}(P Q)}\right) \\
& +\frac{1}{2}\left(\nu_{P Q} \bar{U}_{e, \rho_{e}(P Q)}-\nu_{P Q} \nu_{\rho_{e}(P Q)} \bar{U}_{e, P Q}\right)=0 .
\end{aligned}
$$

Here we have used that $\rho_{e}$ is an involution on $\mathcal{R}_{e}$ and $\nu_{\rho_{e}(P Q)} \nu_{P Q}=1$ from (6.16).

For $(P, Q) \in \mathcal{R}_{h}$, we achieve (6.43) and the first identity in (6.23) by taking

$$
U_{h, P Q}^{\prime}=\frac{1}{2}\left(U_{h, P Q}-\bar{U}_{h, \rho(P Q)}\right), \quad U_{h, P Q}^{\prime \prime}=\frac{1}{2}\left(U_{h, P Q}+\bar{U}_{h, \rho(P Q)}\right) .
$$

For $(P, Q) \in \mathcal{R}_{s+s_{*}}$, we achieve the second identity in (6.23) and (6.44) by taking

$$
U_{s+s_{*}, P Q}^{\prime}=\frac{1}{2}\left(U_{s+s_{*}, P Q}-\bar{U}_{s, \rho(P Q)}\right), \quad U_{s+s_{*}, P Q}^{\prime \prime}=\frac{1}{2}\left(U_{s+s_{*}, P Q}+\bar{U}_{s, \rho(P Q)}\right) .
$$
$\mathcal{R}_{j}$

We have determined coefficients for $U_{j, P Q}^{\prime}, U_{j, P Q}^{\prime \prime}$ with $(P, Q) \in \mathcal{R}_{j}$. Let us set for $(P, Q) \in$

$$
\begin{aligned}
V_{j, Q P}^{\prime \prime} & =\lambda_{j}^{-1} \lambda^{P-Q} U_{j, P Q}^{\prime \prime}, \\
V_{j, Q P}^{\prime} & =V_{j, Q P}-V_{j, Q P}^{\prime \prime} .
\end{aligned}
$$

This fulfills the conditions on $V_{j}^{\prime}$ and $V_{j}^{\prime \prime}$ easily. Note that the first identity means that $\left(U^{\prime \prime}, V^{\prime \prime}\right)$ commutes with $T_{1}$. We have obtained the required formal decomposition.

To prove the convergence, we start with

$$
\lambda_{j}^{-1} \lambda^{P-Q}=\nu_{P Q}= \pm 1
$$

for $(P, Q) \in \mathcal{R}_{j}$. So $\pi$ is indeed an $\mathbf{R}$-linear projection which preserves degrees. Since $\left|\nu_{P Q}\right|=1$, we have that

$$
\left|U_{P Q}^{\prime}\right| \leq \max _{\left(P^{\prime}, Q^{\prime}\right)}\left|U_{P^{\prime} Q^{\prime}}\right|
$$

Here $\left(P^{\prime}, Q^{\prime}\right)$ runs over all permutations of $(P, Q)$ in $2 p$ coordinates. The same holds for $V^{\prime}$. Hence, with the notation of Lemma 4.3, we have

$$
\{\pi(U, V)\}_{\text {sym }} \prec(U, V)_{\text {sym }} .
$$

The existence and uniqueness as well as the convergence also follow from Lemma 4.3 ,

We now consider the second case of $\mathcal{C}\left(\mathcal{T}_{1}, \mathcal{T}_{2}, \rho\right)$ by minor changes. Let us define a projection $\pi:\left(\left(\widehat{\mathfrak{M}}_{n}^{2}\right)^{n} \rightarrow \hat{H}\right.$. Here $\hat{H}$ is the space associated with the mappings satisfying the normalized conditions (6.46) $-(\underline{6.49})$. Let $\hat{G}=(\mathrm{I}-\pi) \hat{H}$. We decompose as above

$$
(U, V)=\left(U^{\prime}+U^{\prime \prime}, V^{\prime}+V^{\prime \prime}\right), \quad \pi(U, V)=\left(U^{\prime}, V^{\prime}\right)
$$


Recalling that $\iota_{e}=\left(A_{e}, B_{e}\right) \circ \rho_{e}$ is an involution on $\mathcal{N}_{e}$, we choose :

$$
\begin{array}{cc}
U_{j, P Q}^{\prime \prime}=\frac{1}{2}\left(U_{j, P Q}+\bar{U}_{j, \rho_{j}(P Q)}\right), & (P, Q) \in \mathcal{N}_{h}, \\
U_{j, P Q}^{\prime}=\frac{1}{2}\left(U_{j, P Q}-\bar{U}_{j, \rho_{j}(P Q)}\right), & (P, Q) \in \mathcal{N}_{h}, \\
U_{e, P Q}^{\prime \prime}=\frac{1}{2}\left(U_{e, P Q}+\bar{U}_{e, \iota_{e}(P Q)}\right), & (P, Q) \in \mathcal{N}_{e}, \\
U_{e, P Q}^{\prime}=\frac{1}{2}\left(U_{e, P Q}-U_{e, \iota_{e}(P Q)}\right), & (P, Q) \in \mathcal{N}_{e}, \\
U_{s+s_{*}, P Q}^{\prime \prime}=\frac{1}{2}\left(U_{s+s_{*}, P Q}+\bar{U}_{s, \rho(P Q)}\right), & (P, Q) \in \mathcal{N}_{s+s_{*}}, \\
U_{s+s_{*}, P Q}^{\prime}=\frac{1}{2}\left(U_{s+s_{*}, P Q}-\bar{U}_{s, \rho(P Q)}\right), & (P, Q) \in \mathcal{N}_{s+s_{*}} .
\end{array}
$$

We still use (6.50) for $(P, Q) \notin \mathcal{R}_{j}$. For $(P, Q) \in R_{j}$, define $V_{j, Q P}^{\prime \prime}$ by (6.52) and $V_{j, Q P}^{\prime}=$ $V_{j, Q P}-V_{j, Q P}^{\prime \prime}$, after we set

$$
U_{j, P Q}^{\prime \prime}=\nu_{P Q}^{+} U_{j,\left(A_{j}, B_{j}\right)(P, Q)}^{\prime \prime}, \quad U_{j, P Q}^{\prime}=U_{j, P Q}-U_{j, P Q}^{\prime \prime}, \quad(P, Q) \in \mathcal{R}_{j} \backslash \mathcal{N}_{j} .
$$

Let us verify that $\pi(U, V)=\left(U^{\prime}, V^{\prime}\right)$ is in $\hat{H}$. To verify (6.47) for $j=e$, via (6.56) we compute

$$
U_{e, P Q}^{\prime}+\bar{U}_{e,\left(A_{e}, B_{e}\right) \circ \rho_{e}(P Q)}^{\prime}=\frac{1}{2}\left(U_{e, P Q}-\bar{U}_{e, \iota_{e}(P Q)}\right)+\frac{1}{2}\left(U_{e, \iota_{e}(P Q)}-\bar{U}_{e, P Q}\right)=0 .
$$

We also know that $\rho$ is an involution on $\mathcal{N}_{h}$ and it is a bijection from $\mathcal{N}_{s+s_{*}}$ onto $\mathcal{N}_{s}$. Analogously, we verify (6.48) and (6.49) via (6.56) and (6.60). Note that $(P, Q) \rightarrow\left(A_{j}, B_{j}\right)(P, Q)$ is a projection on $\mathcal{N}_{j}$. Analogously, we verify (6.46) via (6.61). This shows that $\pi(U, V)$ is in $\hat{H}$. We can also verify that $\left(U^{\prime \prime}, V^{\prime \prime}\right)=(\mathrm{I}-\pi)(U, V)$ satisfies the conditions on the centralizer, i.e. it is in $\hat{G}$.

As before, we have

$$
\left|U_{j, P Q}^{\prime}\right|,\left|U_{j, P Q}^{\prime \prime}\right| \leq C \max _{i} \max _{\left(P^{\prime}, Q^{\prime}\right) \text { permutation of }(P, Q)}\left|U_{i, P^{\prime} Q^{\prime}}\right| .
$$

Equations (6.52) lead to the same inequality for $V^{\prime \prime}$ and hence for $V^{\prime}=V-V^{\prime \prime}$. Hence, again the result follows from Lemma 4.3,

\subsection{Convergence of linearizations.}

Proposition 6.6. Assume that the family of involutions $\left\{\mathcal{T}_{1}, \mathcal{T}_{2}, \rho\right\}$ is formally linearizable. Assume further that $\sigma_{1}, \ldots, \sigma_{p}$ defined by (5.1)-(5.2), are linear.

(i) There is a biholomorphic mapping in the centralizer of $\{\mathcal{S}, \rho\}$ which linearizes $\tau_{1}$ and $\tau_{2}$.

(ii) Assume further that $\tau_{1}=T_{1}$ and $\tau_{2}=T_{2}$. Then $\left\{\tau_{11}, \ldots, \tau_{1 p}, \rho\right\}$ is holomorphically linearizable.

Proof. (i) Suppose that $\Psi$ is a formal mapping satisfying

$$
\Psi^{-1} \tau_{1 j} \Psi=T_{1 i_{j}}, \quad \Psi \rho=\rho \Psi
$$


Then $T_{1 j}=(L \Psi) \circ T_{1 i_{j}} \circ(L \Psi)^{-1}$, and $L \Psi$ commutes with $\rho$. Replacing $\Psi$ by $\Psi \circ L \Psi^{-1}$, we may assume that $\Psi$ is tangent to the identity and $i_{j}=j$. We decompose $\Psi=\Psi_{1} \Psi_{0}^{-1}$, where $\Psi_{1}$ is normalized w.r.t. $\mathcal{S}, T_{1}, \rho$ and $\Psi_{0}$ is in the centralizer of $\mathcal{S}, T_{1}, \rho$. Since $\Psi, \Psi_{0}$ commute with the $S_{j}$ 's and $\rho$, then $\Psi_{1}$ commutes with the $S_{j}$ 's and $\rho$ too. We now let $\Psi$ denote $\Psi_{1}$.

To be more specific, let us write

$$
\tau_{1}:\left\{\begin{array}{l}
\xi_{i}^{\prime}=\lambda_{i} \eta_{i}+f_{i}(\xi, \eta) \quad i=1, \ldots, p \\
\eta_{i}^{\prime}=\lambda_{i}^{-1} \xi_{i}+g_{i}(\xi, \eta) \quad i=1, \ldots, p
\end{array}\right.
$$

and

$$
\Psi: \begin{cases}\xi_{i}^{\prime}=\xi_{i}+U_{i}(\xi, \eta) & i=1, \ldots, p, \\ \eta_{i}^{\prime}=\eta_{i}+V_{i}(\xi, \eta) & i=1, \ldots, p .\end{cases}
$$

Let us write that $\Psi$ conjugates $\tau_{1}$ to

$$
T_{1}: \xi_{i}^{\prime}=\lambda_{i} \eta_{i}, \quad \eta_{i}^{\prime}=\lambda_{i}^{-1} \xi_{i}, \quad i=1, \ldots, p .
$$

We have $\Psi \circ T_{1}=\tau_{1} \circ \Psi$; that is

$$
\begin{gathered}
\lambda_{i} V_{i}-U_{i} \circ T_{1}=-f_{i} \circ \Psi(\xi, \eta) \quad i=1, \ldots, p, \\
\lambda_{i}^{-1} U_{i}-V_{i} \circ T_{1}=-g_{i} \circ \Psi(\xi, \eta) \quad i=1, \ldots, p .
\end{gathered}
$$

Since $\Psi$ is normalized with respect $\left\{\mathcal{S}, T_{1}, \rho\right\}$, it satisfies Definition 6.4 (iii). Since $\Psi$ commutes with each $S_{j}$, then $U_{j, P Q}=V_{j, Q P}=0$ for $(P, Q) \notin \mathcal{R}_{j}$. Since it also commutes with $\rho$, then by (6.23) and (6.43)-(6.44) we obtain $U_{j, P Q}=0$ for $(P, Q) \in \mathcal{R}_{j}$ and $j=$ $h, s, s+s_{*}$.

We need to majorize $U_{e, P Q}, V_{e, Q P}$ for $(P, Q) \in \mathcal{R}_{e}$. By (6.63) and (6.54), we obtain

$$
U_{e, P Q}-\nu_{P Q}^{-1} V_{e, Q P}=-\lambda_{e}\left\{g_{e} \circ \Psi\right\}_{P Q} .
$$

Using (6.24) and (6.45), we obtain $V_{e, Q P}=\bar{U}_{e, \rho_{e}(P Q)}=-\nu_{P Q}^{-1} U_{e, P Q}$, and hence

$$
U_{e, P Q}=-\frac{1}{2} \lambda_{e}\left\{g_{e} \circ \Psi\right\}_{P Q}, \quad V_{e, Q P}=\frac{1}{2} \nu_{P Q} \lambda_{e}\left\{g_{e} \circ \Psi\right\}_{P Q} .
$$

Therefore, we have

$$
\left|V_{e, Q P}\right|,\left|U_{e, P Q}\right| \leq C\left|\left\{g_{j} \circ \Psi\right\}_{P Q}\right|
$$

The above holds for $(P, Q) \in \mathcal{R}_{e}$. It holds trivially for $(P, Q) \notin \mathcal{R}_{e}$. In view of (4.1), we then have

$$
\psi_{\text {sym }} \prec C g_{\text {sym }} \circ \Psi_{\text {sym }}=g_{\text {sym }} \circ\left(I_{\text {sym }}+\psi_{\text {sym }}\right) .
$$

Therefore, $\psi_{\text {sym }}$ is convergent at the origin and so is $\Psi$.

(ii) Assume now that $\sigma=S, \tau_{1}=T_{1}, \tau_{2}=T_{2}$ are linear. Suppose that $\Psi$ linearizes the $\left\{\tau_{i j}\right\}$ and commutes with $\rho$. We decompose $\Psi=\Psi_{1} \Psi_{0}^{-1}$ with $\Psi_{1}$ being normalized w.r.t. $\mathcal{T}_{1}, \mathcal{T}_{2}, \rho$ and with $\Psi_{0}$ being in the centralizer of $\mathcal{T}_{1}, \mathcal{T}_{2}, \rho$. Since $\Psi^{-1} \tau_{i j} \Psi=T_{i j}$, we have $\Psi_{1}^{-1} \tau_{i j} \Psi_{1}=\Psi_{0}^{-1} T_{i j} \Psi_{0}=T_{i j}$. Hence, $\Psi_{1}$ linearizes the $\tau_{i j}$ and is normalized w.r.t $\mathcal{T}_{1}, \mathcal{T}_{2}, \rho$. Since $\Psi, \Psi_{0}$ commute with $\mathcal{S}, T_{1}$ and $\rho$, so does $\Psi_{1}$. 
We recall

$$
T_{1 j}:\left\{\begin{array}{l}
\xi_{j}^{\prime}=\lambda_{j} \eta_{j} \\
\eta_{j}^{\prime}=\lambda_{j}^{-1} \xi_{j} \\
\xi_{k}^{\prime}=\xi_{k}, \quad k \neq j \\
\eta_{k}^{\prime}=\eta_{k}, \quad k \neq j,
\end{array} \quad \tau_{1 j}:\left\{\begin{array}{l}
\xi_{j}^{\prime}=\lambda_{j} \eta_{j}+f_{j j}(\xi, \eta) \\
\eta_{j}^{\prime}=\lambda_{j}^{-1} \xi_{j}+g_{j j}(\xi, \eta) \\
\xi_{k}^{\prime}=\xi_{k}+f_{j k}(\xi, \eta), \quad k \neq j \\
\eta_{k}^{\prime}=\eta_{k}+g_{j k}(\xi, \eta), \quad k \neq j .
\end{array}\right.\right.
$$

Since we have $\Psi \circ T_{1 j}=\tau_{1 j} \circ \Psi$, we obtain the following relations

$$
\left\{\begin{array}{l}
\lambda_{j} V_{j}-U_{j} \circ T_{1 j}=-f_{j j} \circ \Psi \\
\lambda_{j}^{-1} U_{j}-V_{j} \circ T_{1 j}=-g_{j j} \circ \Psi \\
U_{k}-U_{k} \circ T_{1 j}=-f_{j k} \circ \Psi, \quad k \neq j \\
V_{k}-V_{k} \circ T_{1 j}=-g_{j k} \circ \Psi, \quad k \neq j .
\end{array}\right.
$$

Since $\Psi \in \mathcal{C}\left(\mathcal{S}, T_{1}, \rho\right)$, combining (6.23), (6.26) with the normalizing conditions (6.48), (6.49), we find that $U_{j, P Q}=0=V_{j, Q P}$ for $(P, Q) \in \mathcal{N}_{j}$ and $j=h, s, s+s_{*}$. Using $\Psi \rho=\rho \Psi$, we get $V_{e}=\overline{U_{e} \circ \rho}$. By the first equation above, we get

$$
\lambda_{e} \overline{U_{e} \circ \rho} \circ T_{1 e}-U_{e}=-f_{e e} \circ \tau_{1 e} \circ \Psi .
$$

For $(P, Q) \in \mathcal{N}_{e}$, we have $\left(\lambda_{e} U_{e} \circ \rho \circ T_{1 e}\right)_{P Q}=\bar{U}_{e, A_{e} B_{e}\left(\rho_{e}(P Q)\right)}$. By (6.47), we get

$$
U_{e, P Q}=\frac{1}{2}\left\{f_{e, e} \circ \tau_{1 e} \circ \Psi\right\}_{P Q}, \quad V_{e, Q P}=\nu_{P Q} U_{e, P Q}, \quad(P Q) \in \mathcal{N}_{e}
$$

We now majorize $U_{j, P Q}, V_{j, Q P}$ for $(P, Q) \in \mathcal{R}_{j} \backslash \mathcal{N}_{j}$. Fix $(P, Q) \in \mathcal{R}_{k} \backslash \mathcal{N}_{k}$. Start with some $j$ such that $p_{j}<q_{j}$. In the second last identity in (6.64), let us compose on the right by $T_{1 j^{\prime}}$ with $j^{\prime} \neq k, j$ to get

$$
U_{k} \circ T_{1 j^{\prime}}-U_{k} \circ T_{1 j} \circ T_{1 j^{\prime}}=-f_{j, k} \circ \Psi \circ T_{1 j^{\prime}}=f_{j, k} \circ \tau_{1 j^{\prime}} \circ \Psi .
$$

Let $\left\{\ell_{1}, \ldots, \ell_{d}\right\}$ be the set of $i \neq k$ such that $p_{i}<q_{i}$. Composing successively with the $T_{1 l_{j}}$ 's and adding, we get

$$
U_{k}-U_{k} \circ T_{1 \ell_{1}} \circ \cdots T_{1 \ell_{d}}=-\sum_{i=1}^{d} f_{\ell_{i}, k} \circ \tau_{1 \ell_{1}} \circ \cdots \circ \tau_{1 \ell_{i-1}} \circ \Psi .
$$

Hence, if $P Q \in \mathcal{R}_{k} \backslash \mathcal{N}_{k}$, then

$$
U_{k, P Q}=\left\{U_{k} \circ T_{1 \ell_{1}} \circ \cdots \circ T_{1 \ell_{d}}\right\}_{P Q}-\left\{\sum_{i=1}^{d} f_{\ell_{i}, k} \circ \tau_{1 \ell_{1}} \circ \cdots \circ \tau_{1 \ell_{i-1}} \circ \Psi\right\}_{P Q} .
$$

The first term on the right-hand side, $U_{k,\left(A_{k}, B_{k}\right)(P Q)}$, is either zero or majorized by (6.65). The summations have finitely many combinations. This shows that $U_{k} \prec a_{k} \circ \bar{\Psi}$. By (6.26), we obtain $V_{j} \prec\left(U_{j}\right)_{\text {sym }}$. This shows that $(U, V) \prec b \circ\left(I_{\text {sym }}+(U, V)_{\text {sym }}\right)$ for some analytic mapping $b=O(2)$. Using Lemma 4.3, we obtain the convergence of $U_{k}, V_{k}$.

Theorem 6.7. Let $M$ be a germ of analytic submanifold that is an higher order perturbation of a product quadric $Q$ in $\mathbf{C}^{2 p}$. Assume that $M$ satisfies condition $J$ and it is formally equivalent to $Q$. Suppose that each hyperbolic component has an eigenvalue $\mu_{h}$ which is either a root of unity or satisfies the Brjuno condition (7.38) in which $\mathcal{I}=0, \ell=1, n=$ 
$1, \mu_{i}=\mu_{i, j}=\mu_{h}$, and each $\mu_{s}$ is not a root of unity and satisfies the Brjuno condition. Then $M$ is holomorphically equivalent to the product quadric.

Proof. We first apply a theorem (with $\mathcal{I}=0$ ) in Sto15] that linearize simultaneously and holomorphically the $\sigma_{1}, \ldots, \sigma_{p}$. Note that the small divisor condition in this special case is equivalent that each $\mu_{h}$ is either a root of unity or a Brjuno number. Then, we apply successively the two assertions of Proposition 6.6. Hence, in good holomorphic coordinates, $\left\{\tau_{11}, \ldots, \tau_{1 p}, \rho\right\}$ are linear. Then, by Proposition 2.8, the manifold is holomorphically equivalent to the quadric.

As in the case of Theorem 5.3, we can also prove the first part of the above proof by applying Rüssmann's theorem [Rüs02] successively to each $\sigma_{i}$. This is due to the commutativity property and the special type of the linear parts that lead to the relatively simpler relations on $\mathcal{C}\left(S_{i}\right)$ and $\mathcal{C}^{\mathrm{c}}\left(S_{i}\right)$ for each fixed $i$.

\section{EXISTENCE OF ATTACHED COMPLEX MANIFOLDS}

We are interested in complex submanifolds $K$ in $\mathbf{C}^{2 p}$ that intersect the real submanifold $M$ at the origin. Recall that $M$ has real dimension $2 p$. Generically, the origin is an isolated intersection point if $\operatorname{dim} K=p$. Let us consider the situation when the intersection has dimension $p$. Without further restrictions, there are many such complex submanifolds; for instance, we can take a $p$-dimensional totally real and real analytic submanifold $K_{1}$ of $M$. We then let $K$ be the complexification of $K_{1}$. To ensure the uniqueness or finiteness of the complex submanifolds $K$, we therefore introduce the following.

Definition 7.1. Let $M$ be a formal real submanifold of dimension $2 p$ in $\mathbf{C}^{2 p}$. We say that a formal complex submanifold $K$ is attached to $M$ if $K \cap M$ contains at least two germs of totally real and formal submanifolds $K_{1}, K_{2}$ of dimension $p$ that intersect transversally at the origin. Such a pair $\left\{K_{1}, K_{2}\right\}$ are called a pair of asymptotic formal submanifolds of $M$.

Before we present the details, let us describe the main steps to derive the results. We first derive the results at the formal level. We then apply the results of [Pös86] and [Sto15]. The proof of the co-existence of convergent and divergent attached submanifolds will rely on a theorem of Pöschel on stable invariant submanifolds and Siegel's small divisor technique.

We now describe the formal results. When $p=1$, a non-resonant hyperbolic $M$ admits a unique attached formal holomorphic curve [Kli85]. When $p>1$, new situations arise. First, we show that there are obstructions to attach formal submanifolds. However, the formal obstructions disappear when $M$ admits the maximum number of deck transformations and $M$ is non-resonant. These two conditions allow us to express $M$ in an equivalent form (3.4). This equivalent form for $M$, which has not been used so far, will play an essential role in our proof for $p>1$.

We will consider a real submanifold $M$ which is a higher order perturbation of a nonresonant product quadrics. By adapting the proof of Klingenberg [Kli85] to the manifold $M$ (3.4), we will show the existence of a unique attached formal submanifold for a prescribed non-resonance condition. As in [Kli85], we also show that the complexification of $K$ in $\mathcal{M}$ is a pair of invariant formal submanifolds $\mathcal{K}_{1}, \mathcal{K}_{2}$ of $\sigma$. Furthermore, $K$ is convergent if and only if $\mathcal{K}_{1}$ is convergent. 
Let us first recall the values of the Bishop invariants. The types of the invariants play an important role for the existence and the convergence of attached formal complex submanifolds. From (2.22) and (2.26), we recall that

$$
\begin{gathered}
\gamma_{e}^{-1}=\lambda_{e}+\lambda_{e}^{-1}, \quad \gamma_{h}^{-1}=\lambda_{h}+\bar{\lambda}_{h}, \quad \gamma_{s}^{-1}=1+\bar{\lambda}_{s}^{2}, \\
0<\gamma_{e}<1 / 2, \quad \gamma_{h}>1 / 2, \quad \gamma_{s} \in(-\infty, 1 / 2)+i(0, \infty), \quad \gamma_{s+s_{*}}=1-\bar{\gamma}_{s} .
\end{gathered}
$$

Here we exclude the case that $\operatorname{Re} \gamma_{s}=1 / 2$ or $\gamma_{s}<1 / 2$ as we will assume that $\sigma$ has distinct eigenvalues. We normalize

$$
\begin{gathered}
\lambda_{e}>1, \quad\left|\lambda_{h}\right|=1, \quad\left|\lambda_{s}\right|>1, \quad \lambda_{s+s_{*}}=\bar{\lambda}_{s}^{-1} ; \\
\arg \lambda_{h} \in(0, \pi / 2), \quad \arg \lambda_{s} \in(0, \pi / 2) .
\end{gathered}
$$

Recall that $\mu_{j}=\lambda_{j}^{2}$. By (17.1), we have

$$
\gamma_{j}^{2}=\frac{\mu_{j}}{\left(1+\mu_{j}\right)^{2}}, \quad j=e, h ; \quad \gamma_{s} \bar{\gamma}_{s+s_{*}}=\frac{\bar{\mu}_{s}}{\left(1+\bar{\mu}_{s}\right)^{2}} .
$$

We first verify the following.

Lemma 7.2. Let $\gamma_{j}, \lambda_{j}$ be given by (7.1) (7.4). Let $\mu_{j}=\lambda_{j}^{2}$. Assume that $\mu_{1}, \mu_{1}^{-1}, \ldots$, $\mu_{p}, \mu_{p}^{-1}$ are distinct. Then $\gamma_{e}^{2}, \gamma_{h}^{2}, \bar{\gamma}_{s} \gamma_{s+s_{*}}, \gamma_{s} \bar{\gamma}_{s+s_{*}}$ are distinct $p$ numbers. The latter is equivalent to $\gamma_{1}, \ldots, \gamma_{p}$ being distinct.

Proof. Note that $x^{-1}+x$ and $x^{-1}$ decrease strictly on $(0,1)$. So $\gamma_{e}^{2}, \gamma_{h}^{2}$ are distinct. We also have $\gamma_{s} \bar{\gamma}_{s+s_{*}}=\gamma_{s}-\gamma_{s}^{2}$. If $a, b$ are complex numbers, then $a-a^{2}=b-b^{2}$ if and only if $a=b$ or $a+b=1$. Since $\gamma_{s}$ is not real, then $\gamma_{s} \bar{\gamma}_{s+s_{*}}$ are different from $\gamma_{e}^{2}$ and $\gamma_{h}^{2}$. For any distinct complex numbers $a_{1}, a_{2}$ in $(-\infty, 1 / 2)+i(0, \infty)$. We have $1-a_{2} \neq 1-a_{1}, a_{1}, a_{2}$. The lemma is proved.

Let us first investigate the numbers of pairs of formal asymptotic submanifolds and attached formal submanifolds.

Lemma 7.3. Let $M$ be a formal submanifold that is a third order perturbation of a product quadric $Q$ in $\mathbf{C}^{2 p}$. Assume that $M$ has distinct eigenvalues

$$
\mu_{1}, \ldots, \mu_{p}, \quad \mu_{1}^{-1}, \ldots, \mu_{p}^{-1} .
$$

(i) If $M$ admits an attached formal submanifold, its $C R$ singularity has no elliptic component.

(ii) If $Q$ has no elliptic components, then $Q$ has at least $2^{h_{*}+s_{*}-1}$ pairs of asymptotic totally real and real analytic submanifolds that are contained in a single attached complex submanifold.

(iii) There is no formal submanifold attached to

$$
M: z_{3}=\left(z_{1}+2 \gamma_{1} \bar{z}_{1}\right)^{2}+\left(z_{2}+2 \gamma_{2} \bar{z}_{2}\right)^{3}, \quad z_{4}=\left(z_{2}+2 \gamma_{2} \bar{z}_{2}\right)^{2} .
$$

Here $M$ has a hyperbolic complex tangent at the origin.

(iv) Assume that $M$ has no elliptic component and it admits the maximum number of formal deck transformations. Given $\epsilon_{h}, \epsilon_{s}= \pm 1$, let $\nu=\nu_{\epsilon}:=\left(\nu_{1}, \ldots, \nu_{p}\right)$ with

$$
\nu_{h}:=\mu_{h}^{\epsilon_{h}}, \quad \nu_{s}:=\bar{\mu}_{s}^{\epsilon_{s}}, \quad \nu_{s_{*}+s}:=\mu_{s}^{-\epsilon_{s}}
$$


Suppose that

$$
\nu^{Q} \neq \nu_{j}^{-1}, \quad \forall Q \in \mathbf{N}^{p}, \quad|Q|>0, \quad 1 \leq j \leq p .
$$

Then $M$ admits a unique pair of asymptotic formal submanifolds $K_{1}, K_{2}$ such that each $K_{i}$ is defined by $z^{\prime}=\rho_{i}\left(z^{\prime}\right)$ for a formal anti-holomorphic involution $\rho_{i}$ and the linear part of $\bar{\rho}_{2}^{-1} \bar{\rho}_{1}$ has eigenvalues $\nu_{1}, \ldots, \nu_{p}$. In particular, if (7.7) holds for each $\nu$ of the form (7.6) then $M$ admits exactly $2^{h_{*}+s_{*}-1}$ pairs of asymptotic formal submanifolds.

Proof. (i) Let $M$ be defined by

$$
z_{p+j}=Q_{j}\left(z^{\prime}, \bar{z}^{\prime}\right)+H_{j}\left(z^{\prime}, \bar{z}^{\prime}\right), \quad 1 \leq j \leq p
$$

where $H_{j}\left(z^{\prime}, \overline{z^{\prime}}\right)=O\left(\left|z^{\prime}\right|^{3}\right)$ and each $Q_{j}$ is quadratic. Let $\left\{K_{1}, K_{2}\right\}$ be a pair of asymptotic formal submanifolds of $M$ intersecting a formal complex submanifold $K$. We know that the totally real spaces $T_{0} K_{1}, T_{0} K_{2}$ are contained in $T_{0} M$, the $z^{\prime}$-subspace. Let $K_{i}^{\prime}$ be the projection of $K_{i}$ onto the $z^{\prime}$-subspace, then $K_{1}^{\prime}, K_{2}^{\prime}$ are still totally real. Let $K_{1}^{\prime}$ be defined by

$$
K_{1}^{\prime}: \bar{z}^{\prime}=\mathbf{A} z^{\prime}+R\left(z^{\prime}\right), \quad \overline{\mathbf{A}} \mathbf{A}=\mathbf{I}, \quad R\left(z^{\prime}\right)=O(2)
$$

such that $\rho_{1}\left(z^{\prime}\right):=\overline{\mathbf{A}} \bar{z}^{\prime}+\bar{R}\left(\bar{z}^{\prime}\right)$ defines an anti-holomorphic formal involution. Let $K_{2}$ be the (formal) fixed-point set of the anti-holomorphic involution $\rho_{2}\left(z^{\prime}\right)=\overline{\tilde{\mathbf{A}}} \bar{z}^{\prime}+\overline{\tilde{R}\left(z^{\prime}\right)}$ with $\tilde{R}\left(z^{\prime}\right)=O(2)$. Then $K_{1}, K_{2}$ intersect transversally at the origin if and only if $\operatorname{det}(\tilde{\mathbf{A}}-\mathbf{A}) \neq$ 0 . Let us define holomorphic mappings

$$
\bar{\rho}_{i}\left(z^{\prime}\right): \overline{\rho_{i}\left(z^{\prime}\right)}, \quad i=1,2 .
$$

Then $K$ is given by

$$
z_{p+j}^{\prime \prime}=Q_{j}\left(z^{\prime}, \bar{\rho}_{i}\left(z^{\prime}\right)\right)+H_{j}\left(z^{\prime}, \bar{\rho}_{i}\left(z^{\prime}\right)\right), \quad i=1,2, \quad j=1, \ldots, p .
$$

The two equations agree, if and only if

$$
Q_{j}\left(z^{\prime}, \bar{\rho}_{1}\left(z^{\prime}\right)\right)+H_{j}\left(z^{\prime}, \bar{\rho}_{1}\left(z^{\prime}\right)\right)=Q_{j}\left(z^{\prime}, \bar{\rho}_{2}\left(z^{\prime}\right)\right)+H_{j}\left(z^{\prime}, \bar{\rho}_{2}\left(z^{\prime}\right)\right), \quad 1 \leq j \leq p .
$$

Then the asymptotic totally real submanifolds $\left\{K_{1}, K_{2}\right\}$ are defined by

$$
K_{i}: z_{p+j}=Q_{j}\left(z^{\prime}, \bar{z}^{\prime}\right)+H_{j}\left(z^{\prime}, \bar{z}^{\prime}\right), \quad 1 \leq j \leq p, \quad \rho_{i}\left(z^{\prime}\right)=z^{\prime} .
$$

Recall that

$$
\begin{aligned}
Q_{j}\left(z^{\prime}, \bar{z}\right) & =\left(z_{j}+2 \gamma_{j} \bar{z}_{j}\right)^{2}, \quad j=e, h ; \\
Q_{s}\left(z^{\prime}, \bar{z}^{\prime}\right) & =\left(z_{s+s_{*}}+2 \gamma_{s+s_{*}} \bar{z}_{s}\right)^{2}, \\
Q_{s+s_{*}}\left(z^{\prime}, \bar{z}^{\prime}\right) & =\left(z_{s}+2 \gamma_{s} \bar{z}_{s+s_{*}}\right)^{2} .
\end{aligned}
$$

Let us first find necessary conditions on the linear parts of $\rho_{i}$ for (7.11) to be solvable. Let $w^{\prime}=\mathbf{A} z^{\prime}$ and $\tilde{w}^{\prime}=\tilde{\mathbf{A}} z^{\prime}$. Comparing the quadratic terms in (7.11) for $i=1,2$, we see that

$$
\begin{aligned}
\left(z_{j}+2 \gamma_{j} w_{j}\right)^{2} & =\left(z_{j}+2 \gamma_{j} \tilde{w}_{j}\right)^{2}, \\
\left(z_{s+s_{*}}+2 \gamma_{s+s_{*}} w_{s}\right)^{2} & =\left(z_{s+s_{*}}+2 \gamma_{s+s_{*}} \tilde{w}_{s}\right)^{2}, \\
\left(z_{s}+2 \gamma_{s} w_{s+s_{*}}\right)^{2} & =\left(z_{s}+2 \gamma_{s} \tilde{w}_{s+s_{*}}\right)^{2} .
\end{aligned}
$$


Here $\gamma_{s+s_{*}}=1-\bar{\gamma}_{s}$, by (7.2). For each $j, w_{j} \neq \tilde{w}_{j}$. Otherwise, the fixed points of $\rho_{1}$ and $\rho_{2}$ do not intersect transversally. Therefore, the above 3 identities can be written as

$$
\begin{aligned}
z_{j}+2 \gamma_{j} w_{j} & =-\left(z_{j}+2 \gamma_{j} \tilde{w}_{j}\right), \\
z_{s+s_{*}}+2 \gamma_{s+s_{*}} w_{s} & =-\left(z_{s+s_{*}}+2 \gamma_{s+s_{*}} \tilde{w}_{s}\right), \\
z_{s}+2 \gamma_{s} w_{s+s_{*}} & =-\left(z_{s}+2 \gamma_{s} \tilde{w}_{s+s_{*}}\right) .
\end{aligned}
$$

In the matrix form, we get

$$
\tilde{\mathbf{A}}=-\gamma^{-1}-\mathbf{A} \text {, with } \quad \gamma:=\left(\begin{array}{cccc}
\gamma_{e_{*}} & \mathbf{0} & \mathbf{0} & \mathbf{0} \\
\mathbf{0} & \gamma_{h_{*}} & \mathbf{0} & \mathbf{0} \\
\mathbf{0} & \mathbf{0} & \mathbf{0} & \gamma_{s_{*}} \\
\mathbf{0} & \mathbf{0} & \tilde{\gamma}_{s_{*}} & \mathbf{0}
\end{array}\right) \text {. }
$$

Here in matrices $\tilde{\gamma}_{s_{*}}=\mathbf{I}_{s_{*}}-\bar{\gamma}_{s_{*}}$. Let us express in block matrices

$$
\mathbf{A}=\left(\begin{array}{cccc}
\mathbf{A}_{e_{*} e_{*}} & \mathbf{A}_{e_{*} h_{*}} & \mathbf{A}_{e_{*} s_{*}} & \mathbf{A}_{e_{*}\left(2 s_{*}\right)} \\
\mathbf{A}_{h_{*} e_{*}} & \mathbf{A}_{h_{*} h_{*}} & \mathbf{A}_{h_{*} s_{*}} & \mathbf{A}_{h_{*}\left(2 s_{*}\right)} \\
\mathbf{A}_{s_{*} e_{*}} & \mathbf{A}_{s_{*} h_{*}} & \mathbf{A}_{s_{*} s_{*}} & \mathbf{A}_{s_{*}\left(2 s_{*}\right)} \\
\mathbf{A}_{\left(2 s_{*}\right) e_{*}} & \mathbf{A}_{\left(2 s_{*}\right) h_{*}} & \mathbf{A}_{\left(2 s_{*}\right) s_{*}} & \mathbf{A}_{\left(2 s_{*}\right)\left(2 s_{*}\right)}
\end{array}\right)
$$

where the diagonal block matrices are of sizes $e_{*} \times e_{*}, h_{*} \times h_{*}, s_{*} \times s_{*}$, and $s_{*} \times s_{*}$, respectively. By $\mathbf{A} \overline{\mathbf{A}}=\mathbf{I}, \tilde{\mathbf{A}} \tilde{\mathbf{A}}=\mathbf{I}$ and $(7.13)$ we get $(\tilde{\mathbf{A}} \overline{\tilde{\mathbf{A}}}-\mathbf{A} \overline{\mathbf{A}}) \bar{\gamma}=0$, i.e. $\boldsymbol{\gamma}^{-1}+\mathbf{A}+\boldsymbol{\gamma}^{-1} \overline{\mathbf{A}} \bar{\gamma}=0$. Recall that $\gamma_{1}^{2}, \ldots, \gamma_{e_{*}+h_{*}}^{2}$ are real and distinct. It is easy to see that $\mathbf{A}_{e_{*} h_{*}}=0, \mathbf{A}_{h_{*} e_{*}}=0$, and $\mathbf{A}_{e_{*} e_{*}}, \mathbf{A}_{h_{*} h_{*}}$ are diagonal. Also,

$$
\mathbf{A}_{e_{*} e_{*}}+\overline{\mathbf{A}}_{e_{*} e_{*}}=-\gamma_{e_{*}}^{-1}, \quad \mathbf{A}_{h_{*} h_{*}}+\overline{\mathbf{A}}_{h_{*} h_{*}}=-\gamma_{h_{*}}^{-1} .
$$

In block matrices, we obtain

$$
\begin{gathered}
\gamma_{j}^{-1} \overline{\mathbf{A}}_{j\left(2 s_{*}\right)} \overline{\tilde{\gamma}}_{s_{*}}=-\mathbf{A}_{j s_{*}}, \quad \tilde{\boldsymbol{\gamma}}_{s_{*}}^{-1} \overline{\mathbf{A}}_{\left(2 s_{*}\right) j} \bar{\gamma}_{j}=-\mathbf{A}_{s_{*} j} ; \\
\gamma_{j}^{-1} \overline{\mathbf{A}}_{j s_{*}} \bar{\gamma}_{s_{*}}=-\mathbf{A}_{j\left(2 s_{*}\right)}, \quad \boldsymbol{\gamma}_{s_{*}}^{-1} \overline{\mathbf{A}}_{s_{*} j} \gamma_{j}=-\mathbf{A}_{\left(2 s_{*}\right) j} ; \\
\tilde{\gamma}_{s_{*}}^{-1} \overline{\mathbf{A}}_{\left(2 s_{*}\right)\left(2 s_{*}\right)} \overline{\tilde{\gamma}}_{s_{*}}=-\mathbf{A}_{s_{*} s_{*}}, \quad \tilde{\boldsymbol{\gamma}}_{s_{*}}^{-1} \overline{\mathbf{A}}_{\left(2 s_{*}\right) s_{*}} \bar{\gamma}_{s_{*}}=-\mathbf{A}_{s_{*}\left(2 s_{*}\right)}-\tilde{\gamma}_{s_{*}}^{-1}, \\
\gamma_{s_{*}}^{-1} \overline{\mathbf{A}}_{s_{*}\left(2 s_{*}\right)} \overline{\tilde{\gamma}}_{s_{*}}=-\mathbf{A}_{\left(2 s_{*}\right) s_{*}}-\gamma_{s_{*}}^{-1}, \quad \gamma_{s_{*}}^{-1} \overline{\mathbf{A}}_{s_{*} s_{*}} \bar{\gamma}_{s_{*}}=-\mathbf{A}_{\left(2 s_{*}\right)\left(2 s_{*}\right)} .
\end{gathered}
$$

In the first 4 equations, we have $j=e_{*}, h_{*}$.

By Lemma 17.2, we know that $\gamma_{e}^{2}, \gamma_{h}^{2}$, and $\gamma_{s} \bar{\gamma}_{s+s_{*}}$ are distinct. Thus, $\mathbf{A}_{j s_{*}}=\mathbf{A}_{j(2 s)_{*}}=\mathbf{0}$ and $\mathbf{A}_{s_{*} j}=\mathbf{A}_{\left(2 s_{*}\right) j}=\mathbf{0}$ for $j=e_{*}, h_{*}$. Since $\gamma_{s} \bar{\gamma}_{s+s_{*}}$ is different from all $\gamma_{s+s_{*}} \bar{\gamma}_{s}$, then $\mathbf{A}_{s_{*} s_{*}}=\mathbf{A}_{\left(2 s_{*}\right)\left(2 s_{*}\right)}=\mathbf{0}$ while $\mathbf{A}_{s_{*}\left(2 s_{*}\right)}, \mathbf{A}_{\left(2 s_{*}\right) s_{*}}$ are diagonal. Now $\mathbf{A} \overline{\mathbf{A}}=\mathbf{I}$ implies that

$$
\mathbf{A}_{e_{*} e_{*}} \overline{\mathbf{A}}_{e_{*} e_{*}}=\mathbf{I}, \quad \mathbf{A}_{h_{*} h_{*}} \overline{\mathbf{A}}_{h_{*} h_{*}}=\mathbf{I}, \quad \mathbf{A}_{s_{*}\left(2 s_{*}\right)} \overline{\mathbf{A}}_{\left(2 s_{*}\right) s_{*}}=\mathbf{I} .
$$

Combining the first identities in (7.14) and (7.19), we know that the diagonal eth element $a_{e}$ of $\mathbf{A}_{e_{*} e_{*}}$ must satisfy $a_{e}+\bar{a}_{e}=-\gamma_{e}^{-1}, a_{e} \bar{a}_{e}=1$. Since $0<\gamma_{e}<1 / 2$, there is no such solution $a_{e}$ if $e_{*}>0$. We have verified (i).

(ii) For the hyperbolic components, by (7.1) we have $\gamma_{h}^{-1}=\lambda_{h}+\bar{\lambda}_{h}$ with $\left|\lambda_{h}\right|=1$ By the second identities in (7.14), (7.19), and by (7.13), we obtain $\left(a_{h}, \tilde{a}_{h}\right)=\left(-\lambda_{h},-\bar{\lambda}_{h}\right)$ or $\left(-\bar{\lambda}_{h},-\lambda_{h}\right)$. For the complex components, we use $\mathbf{A}_{s_{*}\left(2 s_{*}\right)} \overline{\mathbf{A}}_{\left(2 s_{*}\right) s_{*}}=\mathbf{I}$ and multiply the 
second identity in (7.17) by the diagonal matrix $\mathbf{A}_{s_{*}\left(2 s_{*}\right)}$. Thus the $s$ th diagonal element $a_{s}$ of $\mathbf{A}_{s_{*}\left(2 s_{*}\right)}$ satisfies $a_{s}\left(a_{s}+\tilde{\gamma}_{s}^{-1}\right)+\tilde{\gamma}_{s}^{-1} \bar{\gamma}_{s}=0$. By the last identity in (7.5), we get

$$
a_{s}^{2}+\left(1+\mu_{s}^{-1}\right) a_{s}+\mu_{s}^{-1}=0
$$

Obviously $a_{s}=-1,-\mu_{s}^{-1}$ are solutions. By (7.13), we get $\left(a_{s}, \tilde{a}_{s}\right)=\left(-1,1-\tilde{\gamma}_{s}^{-1}\right)=$ $\left(-1,-\mu_{s}^{-1}\right)$ or $\left(-\mu_{s}^{-1}, \mu_{s}^{-1}-\tilde{\gamma}_{s}^{-1}\right)=\left(-\mu_{s}^{-1},-1\right)$. Each tuple determines a tuple $\left(b_{s}, \tilde{b}_{s}\right)$ by (17.19), with $b_{s}$ being the diagonal entries of $\mathbf{A}_{\left(2 s_{*}\right) s_{*}}$. We verify that $\left(b_{s}, \tilde{b}_{s}\right)=\left(\bar{a}_{s}^{-1}, \overline{\tilde{a}}_{s}^{-1}\right)$. There are exactly $2^{h_{*}+s_{*}-1}$ solutions for $\mathbf{A}$ and $\tilde{\mathbf{A}}$ since we can only determine the pairs $\left\{\mathbf{A}_{h_{*} h_{*}}, \tilde{\mathbf{A}}_{h_{*} h_{*}}\right\},\left\{\mathbf{A}_{s_{*}\left(2 s_{*}\right)}, \tilde{\mathbf{A}}_{s_{*}\left(2 s_{*}\right)}\right\}$. Indeed, we have

$$
\mathbf{A}=\left(\begin{array}{ccc}
\operatorname{diag}\left(a_{h}\right) & \mathbf{0} & \mathbf{0} \\
\mathbf{0} & \mathbf{0} & \operatorname{diag}\left(a_{s}\right) \\
\mathbf{0} & \operatorname{diag}\left(b_{s}\right) & \mathbf{0}
\end{array}\right), \quad \tilde{A}=\left(\begin{array}{ccc}
\operatorname{diag}\left(\tilde{a}_{h}\right) & \mathbf{0} & \mathbf{0} \\
\mathbf{0} & \mathbf{0} & \operatorname{diag}\left(\tilde{a}_{s}\right) \\
\mathbf{0} & \operatorname{diag}\left(\tilde{b}_{s}\right) & \mathbf{0}
\end{array}\right)
$$

$$
\begin{aligned}
& \operatorname{diag} \nu:=\tilde{\mathbf{A}}^{-1} \mathbf{A}=\left(\begin{array}{ccc}
\operatorname{diag}\left(\tilde{a}_{h}^{-1} a_{h}\right) & \mathbf{0} & \mathbf{0} \\
\mathbf{0} & \operatorname{diag}\left(\tilde{b}_{s}^{-1} b_{s}\right) & \mathbf{0} \\
\mathbf{0} & \mathbf{0} & \operatorname{diag}\left(\tilde{a}_{s}^{-1} a_{s}\right)
\end{array}\right) \text {, } \\
& \nu=\mu_{\epsilon}=\left(\mu_{h}^{\epsilon_{h}}, \bar{\mu}_{s}^{\epsilon_{s}}, \mu_{s}^{-\epsilon_{s}}\right), \quad \epsilon_{h}^{2}, \epsilon_{s}^{2}=1, \quad \nu_{s+s_{*}}=\bar{\nu}_{s}^{-1},
\end{aligned}
$$

where there are $2^{h_{*}+s_{*}}$ distinct combinations. Thus, we get exactly $2^{h_{*}+s_{*}-1}$ pairs $\left\{K_{\epsilon}^{1}, K_{\epsilon}^{2}\right\}$ of asymptotic linear submanifolds indexed by $\epsilon=\left(\epsilon_{1}, \ldots, \epsilon_{h_{*}+s_{*}}\right)$ with $\epsilon_{j}^{2}=1$ for the product quadric. The attached formal submanifolds associated to these linear asymptotic submanifolds are unique and restricting to $\epsilon_{i}=1$ for all $i$, it is given by

$$
z_{p+h}=\left(1-4 \gamma_{h}^{2}\right) z_{h}^{2}, z_{p+s}=\left(1-2 \gamma_{s+s_{*}}\right)^{2} z_{s+s_{*}}^{2}, z_{p+s+s_{*}}=\left(1-2 \gamma_{s}\right)^{2} z_{s}^{2}
$$

Here we have used $\left(1-4 \gamma_{h}^{2}\right)=\left(1-2 \gamma_{h} \lambda_{h}\right)^{2}$.

In summary, we have shown that there are exactly $2^{h_{*}+s_{*}-1}$ pairs of linear anti-holomorphic involutions $\left\{\rho_{1}, \rho_{2}\right\}$. In (iv) we show that under the non-resonant conditions on $\mu_{1}, \ldots, \mu_{p}$, they are the only pairs of anti-holomorphic involutions. This finishes the proof of (ii).

(iii). Let us continue the computation for the perturbations. We have determined linear parts of antiholomorphic involutions $\rho_{i}$. We expand components of $R\left(z^{\prime}\right)$ as $R_{j}\left(z^{\prime}\right)=$ $\sum_{k=2}^{\infty} R_{j ; k}\left(z^{\prime}\right)$. Here $R_{j ; k}$ are homogeneous terms of degree $k$. We expand $\tilde{R}_{j}$ analogously. Suppose that terms of order up to $k-1$ in $R_{j}, \tilde{R}_{j}$ have been determined. For the hyperbolic components, we need to solve the equations

$$
4 \sqrt{1-4 \gamma_{h}^{2}} z_{h}\left(R_{h ; k}\left(z^{\prime}\right)+\tilde{R}_{h ; k}\left(z^{\prime}\right)\right)=\cdots
$$

where the right-hand side has been determined. Indeed, let us compute the terms of degree $k$ in (7.11) to obtain

$$
\left(1-2 \gamma_{j} \lambda_{j}\right)^{2} z_{j}^{2}+2\left(1-2 \gamma_{j} \lambda_{j}\right) z_{j} R_{j ; k}=\left(1-2 \gamma_{j} \lambda_{j}^{-1}\right)^{2} z_{j}^{2}+2\left(1-2 \gamma_{j} \lambda_{j}^{-1}\right) z_{j} \tilde{R}_{j ; k}+\mathcal{R}
$$

where $\mathcal{R}$ is a polynomial that depends on $\tilde{R}_{j ; l}, R_{j ; l}, l<k$. Since $\left(1-2 \gamma_{j} \lambda_{j}\right)=-\left(1-2 \gamma_{j} \lambda_{j}^{-1}\right)$, we obtain (7.22).

When $p>1$, the system of equations (7.22) cannot be solved even formally, unless the right-hand side is divisible by $z_{h}$. When $p=1$, the equation (7.22) is clearly solvable. 
In fact, under the non-resonant condition on $\mu_{1}$, the formal anti-holomorphic involutions $\left\{\rho_{1}, \rho_{2}\right\}$ can be uniquely determined.

Let us keep the above notation and compute for the example stated in (iii). We need to solve

$$
\begin{aligned}
\left(z_{1}+2 \gamma_{1} \tilde{w}_{1}\right)^{2}+\left(z_{2}+2 \gamma_{2} \tilde{w}_{2}\right)^{3} & =\left(z_{1}+2 \gamma_{1} w_{1}\right)^{2}+\left(z_{2}+2 \gamma_{2} w_{2}\right)^{3}, \\
\left(z_{2}+2 \gamma_{2} \tilde{w}_{2}\right)^{2} & =\left(z_{2}+2 \gamma_{2} w_{2}\right)^{2} .
\end{aligned}
$$

Again $\tilde{w}_{2}-w_{2}$ cannot be identically zero. Thus $\tilde{w}_{2}=-w_{2}-\gamma_{2}^{-1} z_{2}$. Then we need to solve

$$
\left(z_{1}+2 \gamma_{1} \tilde{w}_{1}\right)^{2}=\left(z_{1}+2 \gamma_{1} w_{1}\right)^{2}+2\left(z_{2}+2 \gamma_{2} w_{2}\right)^{3} .
$$

By (ii), we know that $w_{1}=-\lambda_{1} z_{1}+R_{1}\left(z^{\prime}\right)$ and $w_{2}=-\lambda_{2} z_{2}+R_{2}\left(z^{\prime}\right)$ with $R_{i}\left(z^{\prime}\right)=O(2)$. Also $\tilde{w}_{1}=-\bar{\lambda}_{1} z_{1}+\tilde{R}_{1}\left(z^{\prime}\right)$ and $\tilde{w}_{2}=-\bar{\lambda}_{2} z_{2}+\tilde{R}_{2}\left(z^{\prime}\right)$. Comparing the cubic terms implies that $z_{1}$ must divide $2\left(1-2 \gamma_{2} \lambda_{2}\right)^{3} z_{2}^{3}$, which is a contradiction.

(iv) For a general $M$, following Klingenberg [Kli85] we reformulate the problem by considering the following equations

$$
\begin{array}{rlrl}
h\left(z^{\prime}\right) & =Q\left(z^{\prime}, \bar{\rho}_{i}\left(z^{\prime}\right)\right)+H\left(z^{\prime}, \bar{\rho}_{i}\left(z^{\prime}\right)\right), & & i=1,2, \\
h^{*}\left(\bar{\rho}_{i}\left(z^{\prime}\right)\right) & =\bar{Q}\left(\bar{\rho}_{i}\left(z^{\prime}\right), z^{\prime}\right)+\bar{H}\left(\bar{\rho}_{i}\left(z^{\prime}\right), z^{\prime}\right), & i=1,2 .
\end{array}
$$

Here $h, h^{*}, \bar{\rho}_{i}$ are unknowns. Initially, we require that $\bar{\rho}_{1}, \bar{\rho}_{2}$ be arbitrary biholomorphic maps, except their linear parts match with $z^{\prime} \rightarrow A z^{\prime}$ and $z^{\prime} \rightarrow \tilde{A} z^{\prime}$. This will ensure that the solutions $\bar{\rho}_{i}$ are unique and they are involutions.

As demonstrated in (iii), in general there is no formal submanifold attached to $M$. Thus we assume that $M$ is a higher order perturbation of product quadric without elliptic component and it admits the maximum number of deck transformation.

We may assume that

$$
\begin{aligned}
z_{p+h} & =\left(z_{h}+2 \gamma_{h} \bar{z}_{h}+E_{h}\left(z^{\prime}, \bar{z}^{\prime}\right)\right)^{2}, \\
z_{p+s} & =\left(z_{s}+2 \gamma_{s} \bar{z}_{s+s_{*}}+E_{s}\left(z^{\prime}, \bar{z}^{\prime}\right)\right)^{2}, \\
z_{p+s+s_{*}} & =\left(z_{s+s_{*}}+2 \gamma_{s+s_{*}} \bar{z}_{s}+E_{s+s_{*}}\left(z^{\prime}, \bar{z}^{\prime}\right)\right)^{2} .
\end{aligned}
$$

For late references, we express the above in an abbreviated form:

$$
M \subset \mathbf{C}^{2 p}: z_{p+j}=\left(L_{j}\left(z^{\prime}, \bar{z}^{\prime}\right)+E_{j}\left(z^{\prime}, \bar{z}^{\prime}\right)\right)^{2}, \quad 1 \leq j \leq p .
$$

We fix linear parts of $\rho_{i}$ such that

$$
\rho_{1}\left(z^{\prime}\right)=\bar{A} \bar{z}^{\prime}+\bar{R}\left(\bar{z}^{\prime}\right), \quad \rho_{2}\left(z^{\prime}\right)=\overline{\tilde{A}} \bar{z}^{\prime}+\overline{\tilde{R}}\left(\bar{z}^{\prime}\right) .
$$

For $i=1,2$ we then need to solve $\bar{\rho}_{i}$ from

$$
\begin{aligned}
z_{h}+2 \gamma_{h} \bar{\rho}_{i h}+E_{h}\left(z^{\prime}, \bar{\rho}_{i}\right) & =(-1)^{i} f_{h}\left(z^{\prime}\right), \\
z_{s}+2 \gamma_{s} \bar{\rho}_{i s+s_{*}}+E_{s}\left(z^{\prime}, \bar{\rho}_{i}\right) & =(-1)^{i} f_{s}\left(z^{\prime}\right), \\
z_{s+s_{*}}+2 \gamma_{s+s_{*}} \bar{\rho}_{i s}+E_{s+s_{*}}\left(z^{\prime}, \bar{\rho}_{i}\right) & =(-1)^{i} f_{s+s_{*}}\left(z^{\prime}\right), \\
2 \gamma_{h} z_{h}+\bar{\rho}_{i h}+\bar{E}_{h}\left(\bar{\rho}_{i}, z^{\prime}\right) & =(-1)^{i} f_{h}^{*}\left(\bar{\rho}_{i}\right), \\
2 \bar{\gamma}_{s} z_{s+s_{*}}+\bar{\rho}_{i s}+\bar{E}_{s}\left(\bar{\rho}_{i}, z^{\prime}\right) & =(-1)^{i} f_{s}^{*}\left(\bar{\rho}_{i}\right), \\
2 \bar{\gamma}_{s+s_{*}} z_{s}+\bar{\rho}_{i s+s_{*}}+\bar{E}_{s+s_{*}}\left(\bar{\rho}_{i}, z^{\prime}\right) & =(-1)^{i} f_{s+s_{*}}^{*}\left(\bar{\rho}_{i}\right) .
\end{aligned}
$$


Suppose that we have determined terms of $R_{j}, \tilde{R}_{j}, f_{j}, f_{j}^{*}$ of order $<k$. We have

$$
\bar{\rho}_{1}\left(z^{\prime}\right)=\mathbf{A} z^{\prime}+R\left(z^{\prime}\right), \quad \bar{\rho}_{1}^{-1}\left(z^{\prime}\right)=\mathbf{A}^{-1} z^{\prime}-\mathbf{A}^{-1} R^{\prime}\left(\mathbf{A}^{-1} z^{\prime}\right)
$$

where the terms in $R^{\prime}-R$ of order $k$ depend only on terms of $R$ of order $<k$. For terms of order $k$, by eliminating $f_{j}$, we need to solve

$$
R_{j Q}+\tilde{R}_{j Q}=\cdots
$$

where the dots denote terms which have been determined. We compose from right in the last 3 identities for $i=1$ (resp. $i=2$ ) by $\bar{\rho}_{1}^{-1}\left(\right.$ resp. $\bar{\rho}_{2}^{-1}$ ). Eliminating $f^{*}$ from the new identities, we obtain

$$
\mathbf{A}^{-1} R\left(\mathbf{A}^{-1} z^{\prime}\right)+\tilde{\mathbf{A}}^{-1} \tilde{R}\left(\tilde{\mathbf{A}}^{-1} z^{\prime}\right)=\cdots .
$$

Recall that $\tilde{\mathbf{A}}^{-1} \mathbf{A}=\operatorname{diag} \nu$ with $\nu:=\nu_{\epsilon}$. Multiplying on the left by $\tilde{\mathbf{A}}$, using $\tilde{\mathbf{A}} \mathbf{A}^{-1}=$ $(\operatorname{diag} \nu)^{-1}$, and evaluating at $z^{\prime}=\mathbf{A} \tilde{z}^{\prime}$, we thus need to solve (7.33) and

$$
\nu_{j}^{-1} R_{j, Q}+\nu^{Q} \tilde{R}_{j, Q}=\cdots \text {. }
$$

This shows that $R_{j}, \tilde{R}_{j}$ are uniquely determined as

$$
\nu^{Q} \neq \nu_{j}^{-1}, \quad Q \in \mathbf{N}^{p}, \quad|Q|>1, \quad 1 \leq j \leq p .
$$

To verify that $\rho_{i}$ are involutions, we compose by $\bar{\rho}_{i}^{-1}$ from right in (7.27)-(7.29), and we apply complex conjugate to the coefficients of the new identities. This results in (7.30)-

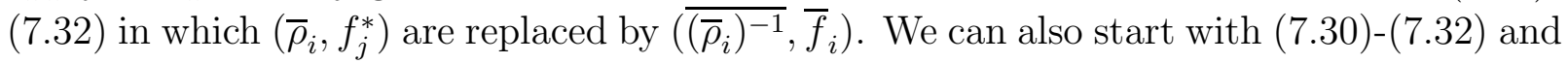
apply the same procedure to get (17.27) $-(\overline{7.29})$, in which $\left(\bar{\rho}_{i}, f_{i}\right)$ are replaced by $\left(\overline{\left(\bar{\rho}_{i}\right)^{-1}}, \bar{f}_{i}^{*}\right)$. By the uniqueness of the solutions, we conclude that $\overline{\left(\bar{\rho}_{i}\right)^{-1}}=\bar{\rho}_{i}$ as both sides have the same linear part. We now have $\overline{\left(\bar{\rho}_{i}\right)^{-1}\left(\bar{z}^{\prime}\right)}=\overline{\rho_{i}\left(z^{\prime}\right)}$. Hence, by $(17.9), \bar{z}^{\prime}=\bar{\rho}_{i}\left(\rho_{i}\left(z^{\prime}\right)\right)=\overline{\rho_{i}^{2}\left(z^{\prime}\right)}$. This shows that each $\rho_{i}$ is an involution.

We now can prove the following theorem.

Theorem 7.4. Let $M$ be a real analytic submanifold in $\mathbf{C}^{2 p}$ defined by (7.26) without elliptic components. Assume that in $(\xi, \eta)$ coordinates, $D \sigma(0)$ is diagonal and has distinct eigenvalues $\mu_{1}, \ldots, \mu_{p}, \mu_{1}^{-1}, \ldots, \mu_{p}^{-1}$. Let $\nu=\nu_{\epsilon}$ be of the form (17.21) and satisfy (7.34). Then $M$ admits a unique pair of formal asymptotic submanifold $\left\{K_{1}^{\epsilon}, K_{2}^{\epsilon}\right\}$ such that the complexification of $K_{1}^{\epsilon}$ in $\mathcal{M}$ is an invariant formal submanifold $\mathcal{H}_{\epsilon}$ of $\sigma$ that is tangent to

$$
\cap_{\epsilon_{j}=1}\left\{\eta_{j}=0\right\} \cap \cap_{\epsilon_{j}=-1}\left\{\xi_{j}=0\right\} .
$$

Furthermore, the complexification of $K_{2}^{\epsilon}$ equals $\tau_{1} \mathcal{H}_{\epsilon}$.

Proof. Let $K_{i}=K_{i}^{\epsilon}$. We will follow Klingenberg's approach for $p=1$, by using the deck transformations. Suppose that $K$ is an attached formal complex submanifold which intersects with $M$ at two totally real formal submanifolds $K_{1}, K_{2}$. We first embed $K_{1} \cup K_{2}$ into $\mathcal{M}$ as $M$ is embedded into $\mathcal{M}$. Let $\mathcal{K}_{i}$ be the complexification of $K_{i}$ in $\mathcal{M}$. Since $\rho$ fixes $K_{i}$ pointwise, then $\rho \mathcal{K}_{i}=\mathcal{K}_{i}$.

We want to show that $\tau_{1}\left(\mathcal{K}_{1}\right)=\mathcal{K}_{2}$; thus $\mathcal{K}_{i}$ is invariant under $\sigma$. We can see that $\mathcal{K}_{i}$ is defined by

$$
\bar{\rho}_{i}\left(z^{\prime}\right)=w^{\prime}
$$


On $\mathcal{K}_{1}$, by (7.27) and (7.29) we have $\tilde{L}\left(z^{\prime}, w^{\prime}\right)+E\left(z^{\prime}, w^{\prime}\right)=-f\left(z^{\prime}\right)$. The latter defines a complex submanifold of dimension $p$. Thus it must be $\mathcal{K}_{1}$. On $\mathcal{M}$,

$$
\left(\tilde{L}_{j}\left(z^{\prime}, w^{\prime}\right)+E_{j}\left(z^{\prime}, w^{\prime}\right)\right)^{2}=z_{p+j}
$$

are invariant by $\tau_{1}$. Thus each $\tilde{L}_{j}\left(z^{\prime}, w^{\prime}\right)+E_{j}\left(z^{\prime}, w^{\prime}\right)$ is either invariant or skew-invariant by $\tau_{1}$. Computing the linear part, we conclude that they are all skew-invariant by $\tau_{1}$. Hence $\tau_{1}\left(\mathcal{K}_{1}\right)$ is defined by $\tilde{L}\left(z^{\prime}, w^{\prime}\right)+E\left(z^{\prime}, w^{\prime}\right)=f\left(z^{\prime}\right)$, which is the defining equations for $\mathcal{K}_{2}$. We must identify the tangent space of $\mathcal{K}_{1}$ at the origin. Let us verify (7.35) for $\epsilon_{j}=1$ for all $j$, while the general case is analogous. Let $A, S$ be the linear parts of $\bar{\rho}_{1}$ and $\sigma=\tau_{1} \tau_{2}$. Define $e\left(z^{\prime}, w^{\prime}\right)=w^{\prime}-A\left(z^{\prime}\right)$. The tangent space to $\mathcal{K}_{1}$ at the origin is $\left\{e\left(z^{\prime}, w^{\prime}\right)=0\right\}$. From the proof of Lemma 7.3 (ii), the matrix of $A$ is

$$
\mathbf{A}=\left(\begin{array}{ccc}
\operatorname{diag}\left(a_{h}\right) & \mathbf{0} & \mathbf{0} \\
\mathbf{0} & \mathbf{0} & \operatorname{diag}\left(a_{s}\right) \\
\mathbf{0} & \operatorname{diag}\left(b_{s}\right) & \mathbf{0}
\end{array}\right)=\left(\begin{array}{ccc}
-\operatorname{diag}\left(\lambda_{h}\right) & \mathbf{0} & \mathbf{0} \\
\mathbf{0} & \mathbf{0} & \mathbf{- I} \\
\mathbf{0} & \mathbf{- I} & \mathbf{0}
\end{array}\right) .
$$

Thus $e_{h}=w_{h}+\lambda_{h} z_{h}, e_{s}=w_{s}+z_{s+s_{*}}$, and $e_{s+s_{*}}=w_{s+s_{*}}+z_{s}$. Using the formulas (2.20) and (2.24) of $\tau_{1}, \tau_{2}$ when $M$ is the product quadric, we can verify that $e_{s+s_{*}} \circ S=\bar{\mu}_{s} e_{s+s_{*}}, e_{s} \circ S=$ $\mu_{s}^{-1} e_{s}$, and $e_{h} \circ S=\bar{\mu}_{h} e_{h}$. Therefore, $e_{j}\left(z^{\prime}, w^{\prime}\right)=c_{j} \eta_{j}$.

Finally, if $\mathcal{K}_{1}$ is convergent, then (7.36) implies that $\bar{\rho}_{1}$ is convergent. Hence $K_{1}$, the fixed point set of $\rho_{1}$, is convergent.

We now study the convergence of attached formal submanifolds. Let us first recall a theorem of Pöschel [Pös86]. Let $\nu$ and $\epsilon$ be as in (7.21). Define

$$
\omega_{\nu}(k)=\min _{1<|P| \leq 2^{k}, P \in \mathbf{N}^{p}} \min _{1 \leq i \leq p}\left\{\left|\nu^{P}-\nu_{i}\right|,\left|\nu^{P}-\nu_{i}^{-1}\right|\right\} .
$$

Suppose that

$$
-\sum \frac{\log \omega_{\nu}(k)}{2^{k}}<\infty
$$

Then the unique invariant formal submanifold of $\sigma$ that is tangent to the $\mathcal{H}_{\epsilon}$ defined by (7.35) is convergent.

We now obtain a consequence of Theorem 7.4 and Pöschel's theorem.

Theorem 7.5. Let $M$ be as in Theorem 7.4. Let $\nu=\mu_{\epsilon}$ be given by (7.21). Assume that $\nu=\left(\mu_{1}^{\epsilon_{1}}, \ldots, \mu_{p}^{\epsilon_{p}}\right)$ satisfy (7.37). Then $M$ admits an attached complex submanifold.

To study the convergence of all attached formal manifolds, we use a theorem in Sto15] to conclude simultaneous convergence of all attached formal submanifolds. In fact the conclusion is much more stronger. It is based on the simultaneous linearization of the $\sigma_{j}$ 's on the resonant ideal, i.e. the ideal $\mathcal{I}$ generated by $\xi_{1} \eta_{1}, \ldots, \xi_{p} \eta_{p}$. Define with $D \sigma_{i}(0):=$ $\operatorname{diag}\left(\mu_{i, 1}, \ldots, \mu_{i, n}\right)$

$$
\omega_{\mathcal{S}, \mathcal{I}}(k)=\inf \left\{\max _{1 \leq i \leq l}\left|\mu_{i}^{Q}-\mu_{i, j}\right| \neq 0: 2 \leq|Q| \leq 2^{k}, 1 \leq j \leq n, Q \in \mathbf{N}^{n}, x^{Q} \notin \mathcal{I}\right\}
$$

where $\mu_{i}^{Q}:=\mu_{i, 1}^{q_{1}} \cdots \mu_{i, n}^{q_{n}}$. As in [Sto15], we say that the family $\mathcal{S}$ is Diophantine on $\mathcal{I}$, if the sequence of numbers (7.38) satisfies (7.37). 
Theorem 7.6. Let $M$ be as in Theorem 7.5, given by (7.26). Assume furthermore that $M$ is non resonant. Suppose that $\mathcal{S}$ is Diophantine on $\mathcal{I}$ or that $M$ has an abelian $C R$ singularity of pure complex type. Then all attached formal submanifolds are convergent. Moreover, the complex submanifolds $K_{j}$ attached to pairs of antiholomorphic involutions $\left\{\rho_{j 1}, \rho_{j 2}\right\}$ have the form

$$
K_{j}: z_{p+i}=\left(L_{i}\left(z^{\prime}, \bar{\rho}_{j 1}\left(z^{\prime}\right)\right)+E_{i}\left(z^{\prime}, \bar{\rho}_{j 1}\left(z^{\prime}\right)\right)\right)^{2}, \quad 1 \leq i \leq p .
$$

Proof. When $M$ has an abelian CR singularity of pure complex type, from the normal form of $\tau_{i j}$ in Theorem 5.3 we know that all invariant submanifolds of $\sigma$ that are tangent to (7.35) are convergent. The non-abelian CR singularity case is a consequence of the theorem of simultaneous linearization of the $\sigma_{j}$ 's along the resonant ideal $\mathcal{I}$ [Sto15][theorem 2.1] and Theorem 7.4. Since, in good holomorphic coordinates, $\sigma$ is linear on the zero set of the resonant ideal, the solutions $\left\{\bar{\rho}_{1}, \bar{\rho}_{2}\right\}$ to (7.27)-(7.32) are linear and there are $2^{h_{*}+s_{*}-1}$ pairs $\left\{\bar{\rho}_{j 1}, \bar{\rho}_{j 2}\right\}$ of solutions. The equation (7.39) is derived in (7.10) for a general situation.

\section{REFERENCES}

[AG09] P. Ahern and X. Gong. Real analytic manifolds in $\mathbb{C}^{n}$ with parabolic complex tangents along a submanifold of codimension one. Ann. Fac. Sci. Toulouse Math. (6), 18(1):1-64, 2009.

[Art68] M. Artin. On the solutions of analytic equations. Invent. Math., 5:277-291, 1968.

[BG83] E. Bedford and B. Gaveau. Envelopes of holomorphy of certain 2-spheres in $\mathbf{C}^{2}$. Amer. J. Math., 105(4):975-1009, 1983.

[BHV10] P. Bonckaert, I. Hoveijn, and F. Verstringe. Local analytic reduction of families of diffeomorphisms. J. Math. Anal. Appl., 367(1):317-328, 2010.

[Bis65] E. Bishop. Differentiable manifolds in complex Euclidean space. Duke Math. J., 32:1-21, 1965.

[BK91] E. Bedford and W. Klingenberg. On the envelope of holomorphy of a 2-sphere in $\mathbf{C}^{2}$. J. Amer. Math. Soc., 4(3):623-646, 1991.

[Brj71] A. D. Brjuno. Analytic form of differential equations. I, II. Trudy Moskov. Mat. Obšč., 25:119-262 (1971); ibid. 26 (1972), 199-239, 1971.

[Bur13] V. Burcea. A normal form for a real 2-codimensional submanifold in $\mathbb{C}^{N+1}$ near a CR singularity. Adv. Math., 243:262-295, 2013.

[Car32] É. Cartan. Sur la géométrie pseudo-conforme des hypersurfaces de l'espace de deux variables complexes II. Ann. Scuola Norm. Sup. Pisa Cl. Sci. (2), 1(4):333-354, 1932.

[Car33] É. Cartan. Sur la géométrie pseudo-conforme des hypersurfaces de l'espace de deux variables complexes. Ann. Mat. Pura Appl., 11(1):17-90, 1933.

[Cha86] M. Chaperon. Géométrie différentielle et singularités de systèmes dynamiques. Astérisque, (138139):1-440, 1986.

[Chi89] E. M. Chirka. Complex analytic sets, volume 46 of Mathematics and its Applications (Soviet Series). Kluwer Academic Publishers Group, 1989. Translated from the Russian by R. A. M. Hoksbergen.

[CM74] S. S. Chern and J. K. Moser. Real hypersurfaces in complex manifolds. Acta Math., 133:219-271, 1974.

[Cof06] A. Coffman. Analytic stability of the CR cross-cap. Pacific J. Math., 226(2):221-258, 2006.

[Gon94] X. Gong. On the convergence of normalizations of real analytic surfaces near hyperbolic complex tangents. Comment. Math. Helv., 69(4):549-574, 1994.

[Gon96] X. Gong. Divergence of the normalization for real Lagrangian surfaces near complex tangents. Pacific J. Math., 176(2):311-324, 1996.

[Gon04] X. Gong. Existence of real analytic surfaces with hyperbolic complex tangent that are formally but not holomorphically equivalent to quadrics. Indiana Univ. Math. J., 53(1):83-95, 2004. 
[GS15] X. Gong and L. Stolovitch. Real submanifolds of maximum complex tangent space at a CR singular point, II. submitted., 66 pages, 2015.

[Gun90] R.C. Gunning. Introduction to holomorphic functions of several variables. Vol. II. Local theory. The Wadsworth \& Brooks/Cole Mathematics Series. Wadsworth \& Brooks/Cole Advanced Books \& Software, Monterey, CA, 1990.

[HK95] X. Huang and S.G. Krantz. On a problem of Moser. Duke Math. J., 78(1):213-228, 1995.

[Hua98] X. Huang. On an $n$-manifold in $\mathbf{C}^{n}$ near an elliptic complex tangent. J. Amer. Math. Soc., 11(3):669-692, 1998.

[HY09a] X. Huang and W. Yin. A Bishop surface with a vanishing Bishop invariant. Invent. Math., 176(3):461-520, 2009.

[HY09b] X. Huang and W. Yin. A codimension two CR singular submanifold that is formally equivalent to a symmetric quadric. Int. Math. Res. Not. IMRN, (15):2789-2828, 2009.

[HY12] X. Huang and W. Yin. Flattening of a CR singular point in a codimension two real submanifold in $\mathbb{C}^{n+1}, 2012$. arXiv:1210.5146 [math.CV].

[Kli85] W. Jr. Klingenberg. Asymptotic curves on real analytic surfaces in $\mathbf{C}^{2}$. Math. Ann., 273(1):149$162,1985$.

[KW82] C.E. Kenig and S.M. Webster. The local hull of holomorphy of a surface in the space of two complex variables. Invent. Math., 67(1):1-21, 1982.

[KW84] C.E. Kenig and S.M. Webster. On the hull of holomorphy of an n-manifold in $\mathbf{C}^{n}$. Ann. Scuola Norm. Sup. Pisa Cl. Sci. (4), 11(2):261-280, 1984.

[Mos56] J. Moser. The analytic invariants of an area-preserving mapping near a hyperbolic fixed point. Comm. Pure Appl. Math., 9:673-692, 1956.

[Mos85] J. Moser. Analytic surfaces in $\mathbf{C}^{2}$ and their local hull of holomorphy. Ann. Acad. Sci. Fenn. Ser. A I Math., 10:397-410, 1985.

[MW83] J. Moser and S.M. Webster. Normal forms for real surfaces in $\mathbf{C}^{2}$ near complex tangents and hyperbolic surface transformations. Acta Math., 150(3-4):255-296, 1983.

[Pös86] J. Pöschel. On invariant manifolds of complex analytic mappings near fixed points. Exposition. Math., 4(2):97-109, 1986.

[Rüs02] H. Rüssmann. Stability of elliptic fixed points of analytic area-preserving mappings under the bruno condition. Ergodic Theory Dynam. Systems, 22(5):1551-1573, 2002.

[Sto00] L. Stolovitch. Singular complete integrability. Inst. Hautes Études Sci. Publ. Math., (91):133-210 (2001), 2000.

[Sto05] L. Stolovitch. Normalisation holomorphe d'algèbres de type Cartan de champs de vecteurs holomorphes singuliers. Ann. of Math. (2), 161(2):589-612, 2005.

[Sto07] L. Stolovitch. Family of intersecting totally real manifolds of $\left(\mathbb{C}^{n}, 0\right)$ and CR-singularities, 2007. preprint, p.1-30.

[Sto15] L. Stolovitch. Family of intersecting totally real manifolds of $\left(\mathbb{C}^{n}, 0\right)$ and germs of holomorphic diffeomorphisms, 2013. Bull. Soc. math. France, 143(2):247-263, 2015.

[Tan62] N. Tanaka. On the pseudo-conformal geometry of hypersurfaces of the space of $n$ complex variables. J. Math. Soc. Japan, 14:397-429, 1962.

[Web92] S. M. Webster. Holomorphic symplectic normalization of a real function. Ann. Scuola Norm. Sup. Pisa Cl. Sci. (4), 19(1):69-86, 1992.

Department of Mathematics, University of Wisconsin, Madison, Wi 53706, U.S.A.

E-mail address: gong@math.wisc.edu

CNRS and Laboratoire J.-A. Dieudonné U.M.R. 6621, Université de Nice - Sophia Antipolis, Parc Valrose 06108 Nice Cedex 02, France.

E-mail address: stolo@unice.fr 